\title{
Quantum hyperentanglement and its applications in quantum information processing*
}

\author{
Fu-Guo Deng ${ }^{a, \dagger}$, Bao-Cang Ren ${ }^{b, \ddagger}$, and Xi-Han $\mathrm{Li}^{c, d, \S}$ \\ a Department of Physics, Applied Optics Beijing Area Major Laboratory, \\ Beijing Normal University, Beijing 100875, China \\ ${ }^{b}$ Department of Physics, Capital Normal University, Beijing 100048, China \\ ${ }^{c}$ Department of Physics, Chongqing University, Chongqing 400044, China \\ $d$ Department of Physics and Computer Science, \\ Wilfrid Laurier University, Waterloo ON N2L 3C5, Canada
}

(Dated: January 3, 2017)

\begin{abstract}
Hyperentanglement is a promising resource in quantum information processing with its high capacity character, defined as the entanglement in multiple degrees of freedom (DOFs) of a quantum system, such as polarization, spatial-mode, orbit-angular-momentum, time-bin and frequency DOFs of photons. Recently, hyperentanglement attracts much attention as all the multiple DOFs can be used to carry information in quantum information processing fully. In this review, we present an overview of the progress achieved so far in the field of hyperentanglement in photon systems and some of its important applications in quantum information processing, including hyperentanglement generation, complete hyperentangled-Bell-state analysis, hyperentanglement concentration, and hyperentanglement purification for high-capacity long-distance quantum communication. Also, a scheme for hyper-controlled-not gate is introduced for hyperparallel photonic quantum computation, which can perform two controlled-not gate operations on both the polarization and spatial-mode DOFs and depress the resources consumed and the photonic dissipation.
\end{abstract}

Keywords: Quantum hyperentanglement, high-capacity quantum communication, concentration and purification, hyperparallel photonic quantum computation, quantum information processing

\section{INTRODUCTION}

Quantum information processing (QIP) has attracted considerable interest and attention of scientists in a variety of disciplines with its ability for improving the methods of dealing and transmitting information [1, 2]. Entanglement is a distinctive feature of quantum physics [3], and it is very useful in QIP, including both quantum communication and quantum computation. Entangled photon systems are the natural resource for establishing quantum channel in long-distance quantum communication, especially in quantum repeaters [4] for some important tasks of communication, such as quantum key distribution [5-7], quantum secret sharing [8], and quantum secure direct communication [9 13. In experiment, the entangled photon systems are usually prepared by the spontaneous parametric down-conversion (SPDC) process in nonlinear crystal [14 16]. In the conventional protocols for quantum information processing, the entanglement in one degree of freedom (DOF) of photon systems is selected in the SPDC process. In fact, there are more than one DOF in a quantum system, such as the polarization, spatial-mode, orbit-angular-momentum, frequency, and time-bin DOFs in a photon system.

Hyperentanglement, the simultaneous entanglement in

\footnotetext{
*Published in Science Bulletin 62(1), 46-68 (2017).

† email: fgdeng@bnu.edu.cn

‡email: renbaocang@cnu.edu.cn

$\S$ email: xihanlicqu@gmail.com
}

multiple DOFs of a quantum system, has been studied extensively in recent years. It is a promising candidate for QIP with its high-capacity character. In experiment, hyperentanglement can be generated by the combination of the techniques used for creating entanglement in a single DOF [17]. With this method, many different types of hyperentangled states can be prepared [18 25], such as the polarization-spatial hyperentangled state [18], polarization-spatial-time-energy hyperentangled state [19], and so on. Hyperentanglement is a fascinating resource for quantum communication and quantum computation. On one hand, it can assist us to implement many important tasks in quantum communication with one DOF of photons, such as quantum dense coding with linear optics [26], the complete Bell-state analysis for the quantum states in the polarization DOF 27 31], the deterministic entanglement purification [31 34], and the efficient quantum repeater 35]. On the other hand, hyperentanglement can be used directly in some important applications in QIP. For example, it can improve the channel capacity of quantum communication and speedup quantum computation largely.

In the applications of hyperentanglement, the complete hyperentangled-Bell-state analysis (HBSA) [36 45], hyper-teleportation of quantum state with more than one DOF [36], hyperentanglement swapping [37], hyperentanglement concentration [46 53], hyperentanglement purification [47, 54 57], and universal entangling quantum gates for hyperparallel photonic quantum computation [58 62] are very useful and important. HBSA is the prerequisite for high-capacity quantum communication protocols with hyperentanglement and it is used to 
distinguish the hyperentangled states. Also, in the practical application of hyperentanglement in quantum communication, the hyperentangled photon systems are produced locally, which leads to the decoherence of the hyperentanglement when the photons are distributed over a channel with environment noise or stored in practical quantum devices. Quantum repeater is a necessary technique to overcome the influence on quantum communication from this decoherence [4]. In high-capacity quantum repeater with hyperentanglement, hyperentanglement concentration and hyperentanglement purification are two passive ways to recover the entanglement in nonlocal hyperentangled photon systems. They are not only useful but also absolutely necessary in longdistance high-capacity quantum communication with hyperentanglement as the self-error-rejecting qubit transmission scheme [63] do not work in depressing the influence of noise from both a long-distance channel and the storage devices for quantum states. Moreover, quantum repeaters for long-distance quantum communication require the entangled photons with higher fidelity (usually $\sim 99 \%$ ) beyond that from faithful qubit transmission schemes.

Different from conventional parallel quantum computation in which the states of quantum systems in one DOF or equivalent are used to encode information, hyperparallel photonic quantum computation performs universal quantum gate operations on two-photon or multiphoton systems by encoding all the quantum states of each photon in multiple DOFs (two or more DOFs) as information carriers [58 62]. With hyperparallel photonic quantum logic gates, the resource consumption can be reduced largely and the photonic dispassion noise can be depressed in quantum circuit [60]. Moreover, the multiple-photon hyperentangled state can be prepared and measured with less resource and less steps by using the hyperparallel photonic quantum logic gates, which may speedup the quantum algorithm [58, [59].

In this review, we will overview the development of hyperentanglement and its applications in QIP in recent several years. We will first review the preparation of hyperentanglement, and then introduce the applications of the hyperentanglement in quantum communication, including hyper-teleportation of an unknown quantum state in more than two DOFs and hyperentanglement swapping. We also highlight how to improve the entanglement of nonlocal hyperentangled photon systems with hyperentanglement concentration and hyperentanglement purification. At last, the principle of a polarization-spatial hyper-controlled-not (hyper-CNOT) gate is described for hyperparallel quantum computing.

\section{PREPARATION OF HYPERENTANGLEMENT}

Hyperentangled states offer significant advantages in QIP due to the presence of quantum correlations in mul- tiple DOFs. In this section, we will introduce the preparation of hyperentangled states of photon systems. In the first part, we overview the preparation of entangled photon pairs with the SPDC process in nonlinear crystals. In the second part, we overview the preparation of hyperentangled photon systems with the combination of the techniques used for creating entanglement in single DOF.

\section{A. Preparation of entanglement in single DOF}

Generally speaking, the most extensive method used to generate an entangled state is the SPDC process in a nonlinear crystal. When a pump laser beam $p$ shines a nonlinear birefringent crystal, the idler photon $i$ and the signal photon $s$ are generated probabilistically from the crystal. The maximal probability can be achieved by satisfying two matching conditions. One is the phasematching:

$$
\vec{k}_{p}=\vec{k}_{s}+\vec{k}_{i}
$$

and the other is energy-matching:

$$
\omega_{p}=\omega_{s}+\omega_{i}
$$

Here $\vec{k}$ represents the wave vector and $\omega$ denotes the frequency. Usually, there are two common kinds of phasematching adopted in experiment, depending on the extraordinary $(e)$ and the ordinary $(o)$ polarizations of the pump photon and the two SPDC photons. The typeI phase-matching is $e \rightarrow o+o$ and the type-II phasematching is $e \rightarrow e+o$.

In the type-I phase-matching, two SPDC photons are both ordinary and have the same polarizations. To generate an entangled state, two crystals with orthogonal optical axes can be used [15]. The principle is shown in Fig. 1. To satisfy the phase-matching condition, two correlated photons are emitted over opposite directions of the cone surface. By selecting one pair of the correlated wavevector modes, the polarization entangled states $\left|\Phi^{ \pm}\right\rangle=\frac{1}{\sqrt{2}}(|H H\rangle \pm|V V\rangle)$ can be prepared. Here $H$ and $V$ represent the horizontal and vertical polarization states of a photon, respectively. An alternative way to prepare an entangled state with type-I phase-matching is using a single crystal and a double passage of the laser beam after reflection on a mirror [16].

In the type-II phase-matching, the two degenerate photons are emitted over two different mutually crossing emission cones [14]. The emission directions of the signal and idler photons are symmetrically oriented with respect to the propagation direction of the pump photon. The two entangled photons are generated along the direction of the intersection of the two cones. Since 


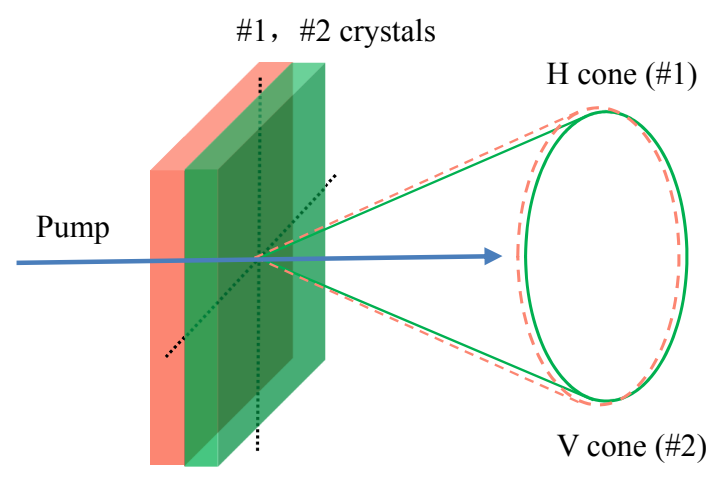

FIG. 1: Type-I polarization entanglement sources [15].

the ordinary and extraordinary photons have orthogonal polarization states, the polarization entangled states $\left|\Psi^{ \pm}\right\rangle=\frac{1}{\sqrt{2}}(|H V\rangle \pm|V H\rangle)$ are prepared with type-II phase-matching. If the two cones only intersect at one point, it is called the collinear SPDC process and orthogonally polarized photons are indistinguishable at exactly this point. The type-II collinear down-conversion is more commonly used in experiment, as it offers a trivial way to deterministically separate the photon pair by their polarization and to work with each photon separately. For the non-collinear type-II SPDC process which is shown is Fig. 2, the two emission cones have two intersection directions, which can be made indistinguishable with respect to their polarization, and then the entangled state is generated.

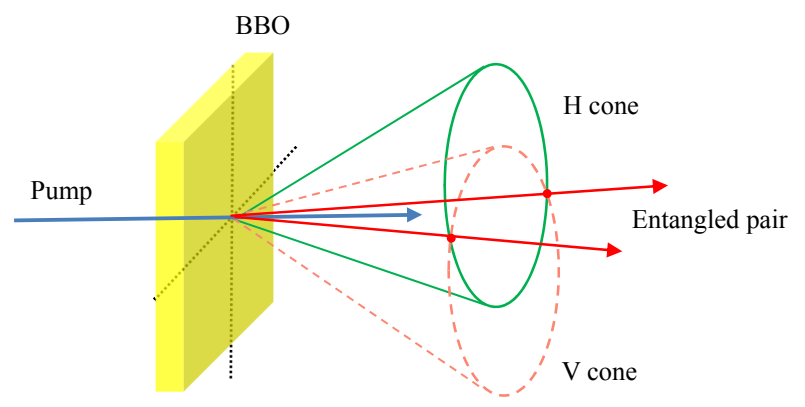

FIG. 2: Non-collinear type-II phase-matching spontaneous parametric down-conversion process 14].

Actually, by obeying these two matching conditions, entanglement in other DOFs can be prepared, such as frequency, time-bin, and spatial-mode DOFs. The SPDC photon pairs are coherently emitted at different emission times as long as the interaction time of the pump wave with the crystal is shorter than the coherence time of the pump photons. The photons are automatically generated into an energy-entangled state due to the nature of the SPDC process. In a word, the energy-time correlations are presented in all SPDC photon pairs. The spatialmode entangled state can be generated by selecting more correlated directions. And if the frequency of the idler and signal photons are not the same, they are always entangled to fulfill the energy-matching condition.

Besides spin, photons possess a further angular momentum, the orbital-angular-momentum (OAM), described by the Laguerre-Gaussian mode $|l, p\rangle$. Under the collinear phase-matching conditions, the OAM of these photons should satisfy $l_{p}=l_{s}+l_{i}$ (consider simple situation $\left.p_{i}=p_{s}=0\right)$. Therefore, when the pump beam is a Gaussian TEM $\mathrm{T}_{00}$ beam, the two generated photons have opposite $l$ as

$$
|\Psi\rangle=\sum_{l=-\infty}^{+\infty} \sqrt{P_{l}}|l\rangle_{s}|-l\rangle_{i},
$$

which is an OAM entangled state. Here $\sqrt{P_{l}}$ denotes the probability of creating a signal photon with OAM $l$ and an idler one with $-l$.

\section{B. Hyperentanglement in more than one degree of freedom}

The techniques used for creating single DOF entanglement can be combined to generate hyperentanglement, which is entangled in more than one DOF in the same time. The first proposal of an energy-momentumpolarization hyperentangled state with a type-II phasematching was presented by Kwiat [17 in 1997. The schematic diagram is shown in Fig. 3. The photons emitted from conjugate points are all energy-time entangled. And the photons generated from 3 and $3^{\prime}$ are automatically in a polarization entangled state since these two cones have opposite polarizations, which are indistinguishable at 3 and $3^{\prime}$. However, these photons have definite momentum. Photons emitted from $1-1^{\prime}-2-2^{\prime}$ are entangled in momentum with the definite polarization state. The quantum state with photons generated along the directions $4-4^{\prime}-5-5^{\prime}$ are entangled in momentum, energy-time, and polarization simultaneously.

In 2005, Yang et al. [18] generated a two-photon state entangled both in polarization and spatial-mode DOFs to realize the all-versus-nothing test of local realism. The setup of generation is shown in Fig. 4. In their experiment, the pump pulse passes through the nonlinear $\mathrm{BBO}$ ( $\beta$-barium borate) crystal twice. The first passage of laser prepares a polarization-entangled pairs $\left|\Psi^{-}\right\rangle_{p}=\frac{1}{\sqrt{2}}\left(|H\rangle_{A}|V\rangle_{B}-|V\rangle_{A}|H\rangle_{B}\right)$ in the spatial modes $L_{A}$ and $R_{B}$ with a small probability. Then the pump beam is reflected by a mirror and goes through the crystal a second time (again). In this time, it probabilistically generates a $\left|\Psi^{-}\right\rangle_{p}$ state in another two path modes $R_{A}$ and $L_{B}$. The generation probabilities of two 


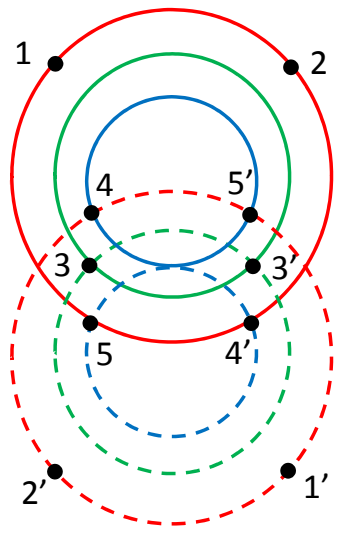

FIG. 3: Schematic diagram of the type-II phase-matching curves. The upper half (solid lines) are three extraordinarypolarized cones with different wavelengths [17]. The ordinarypolarized cones are shown in the lower half (dashed lines) with corresponding wavelength. The numbered points represent the areas of directions to extract hyperentangled states.

passages can be adjusted to equal. Therefore, if there is perfect temporal overlap of modes $R_{A}$ and $L_{A}\left(R_{B}\right.$ and $L_{B}$ ), the two possible ways of producing may interfere, which results a spatial mode entangled state $\left|\Psi^{-}(\phi)\right\rangle_{s}=\frac{1}{\sqrt{2}}\left(|R\rangle_{A}|L\rangle_{B}-\mathrm{e}^{\mathrm{i} \phi}|L\rangle_{A}|R\rangle_{B}\right) . \quad \phi=0$ can be achieved by adjusting the distance between the mirror and the crystal. Then, a maximally hyperentangled state in both polarization and spatial mode $\left(\left|\Psi^{-}\right\rangle_{p} \otimes\left|\Psi^{-}(0)\right\rangle_{s}\right)$ is generated. In their experiment, the generation rate of entangled photon pairs achieves $3.2 \times 10^{4}$ per second.

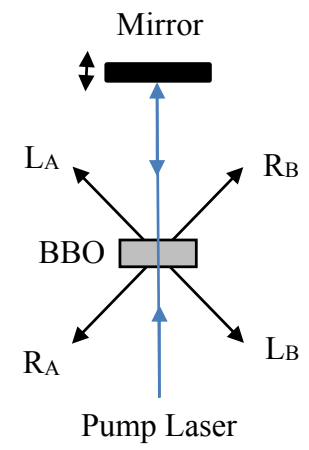

FIG. 4: Schematic diagram of the setup to generate hyperentanglement in both polarization and spatial-mode DOFs [18].

In the same year, an experimental demonstration of a photonic hyperentangled system which simultaneously entangled in polarization, spatial-mode, and time-energy was reported [19]. In their experiment, the entangled pairs are prepared with the type-I phase-matching, and two BBO crystals with orthogonal optical axes are used, which produce pairs of horizontally and vertically polarized photons, respectively. Since the spatial modes emitted from each crystal are indistinguishable, the photon pairs are polarization entangled. Moreover, photon pairs from a single nonlinear crystal are entangled in OAM. And according to the energy-matching condition, each pair is entangled in energy too. The generated state can be written as

$$
\begin{aligned}
& (|H H\rangle+|V V\rangle) \otimes(|+1,-1\rangle+\alpha|0,0\rangle+|-1,+1\rangle) \\
& \otimes(|S S\rangle+|L L\rangle) .
\end{aligned}
$$

Here $| \pm 1\rangle$ and $|0\rangle$ represent the Laguerre-Gauss modes carrying $\pm \hbar$ and 0 OAM, respectively. $|S\rangle$ and $|L\rangle$ denote the relative early and late emission time of photons, respectively. $\alpha$ indicates the OAM spatial-mode balance prescribed by the source and selected via the mode-matching conditions. By collecting only $\pm \hbar$ OAM state, the state in the spatial subspace is also a Bell state. The total dimension of this hyperentangled system is $2 \times 2 \times 3 \times 3 \times 2 \times 2=144$. To verify the quantum correlations, they tested each DOF against the ClauserHorne-Shimony-Holt (CHSH) Bell inequality, and the results showed for each DOF the Bell parameter exceeded the classical limit. They also fully characterized the polarization and spatial-mode state $(2 \otimes 2 \otimes 3 \otimes 3)$ subspace by tomography and obtained the maximum fidelity $F=0.974$.

In 2009, Vallone et al. 20] also realized a two-photon six-qubit hyperentangled state which is entangled in polarization and two longitudinal momentum DOFs. The system used to generate the state consists of two typeI BBO crystal slabs. The polarization entanglement is created by spatially superposing the two perpendicularly polarized emission cones of each crystal. Since the two nonlinear crystal are cut at different phase matching angles, the photon pairs will be created along the surfaces of two cones, called the "internal" $(I)$ and "external" $(E)$ ones. Coherence and indistinguishability between these two emission cones are guaranteed by the coherence length of the pump beam. The double longitudinal momentum entanglement is generated by singling out four pairs of correlated modes with an eight-hole screen, shown in Fig. 5. The hyperentangled state, which is a product of one polarization entanglement and two longitudinal momentum entanglement, can be written as

$$
\begin{gathered}
\frac{1}{\sqrt{2}}\left(|H\rangle_{A}|H\rangle_{B}+\mathrm{e}^{\mathrm{i} \phi_{1}}|V\rangle_{A}|V\rangle_{B}\right) \\
\otimes \frac{1}{\sqrt{2}}\left(|L\rangle_{A}|R\rangle_{B}+\mathrm{e}^{\mathrm{i} \phi_{2}}|R\rangle_{A}|L\rangle_{B}\right) \\
\otimes \frac{1}{\sqrt{2}}\left(|I\rangle_{A}|I\rangle_{B}+\mathrm{e}^{\mathrm{i} \phi_{3}}|E\rangle_{A}|E\rangle_{B}\right) .
\end{gathered}
$$

Here $L$ and $R$ refer to the left and right sides of each cone. The three relative phases $\phi_{1}, \phi_{2}$, and $\phi_{3}$ can be adjusted in experiment. 


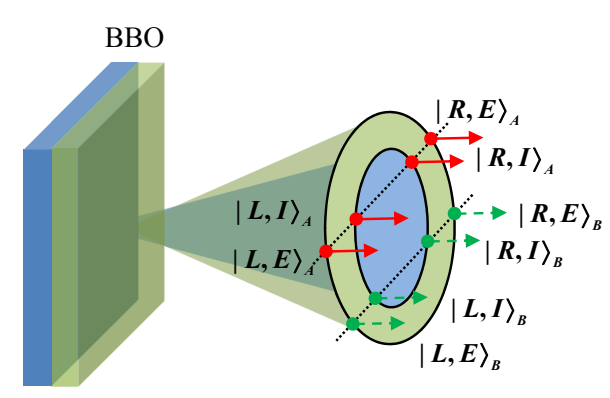

FIG. 5: Schematic diagram of generating hyperentanglement in both polarization and two longitudinal-momentum DOFs [20]. Two BBO crystal slabs are used to generate hyperentangled photon pairs $A$ (solid line) and $B$ (dashed line). $E$ and $I$ denote the external and internal cones, respectively. $R$ and $L$ refer to the left and right sides of each cone, respectively. For simplicity, the positive lens which transforms the conical parametric emission of the crystal into a cylindrical one is not shown in the figure.

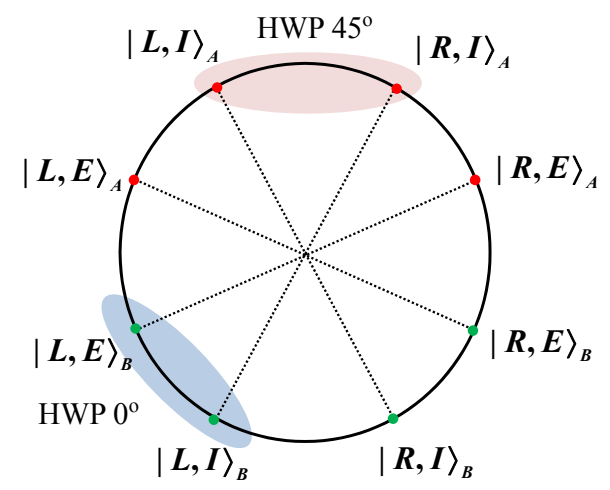

FIG. 6: Model labeling of the hyperentangled state prepared with type-I BBO crystal [21]. To get the six-qubit cluster state, HWPs oriented at $45^{\circ}$ are placed on the $I$ modes of $A$ and HWPs oriented at $0^{\circ}$ are placed on the $L$ modes of $B$.

The hyperentangled states prepared in the previous protocols are the product states of different entangled DOFs. In 2009, Ceccarelli et al. 21] generated a twophoton six-qubit linear cluster state by transforming a two-photon hyperentangled state which is originally entangled in polarization and two linear momentum DOFs. First, they generated the following six-qubit hyperentangled state $\left|\widetilde{H E}_{6}\right\rangle$ by SPDC in a Type-I BBO crystal, shown in Fig. 6 ,

$$
\begin{aligned}
\left|\widetilde{H E}_{6}\right\rangle= & \frac{1}{\sqrt{2}}\left(|E\rangle_{A}|E\rangle_{B}+|I\rangle_{A}|I\rangle_{B}\right) \\
& \otimes \frac{1}{\sqrt{2}}\left(|H\rangle_{A}|H\rangle_{B}-|V\rangle_{A}|V\rangle_{B}\right) \\
& \otimes \frac{1}{\sqrt{2}}\left(|L\rangle_{A}|R\rangle_{B}+|R\rangle_{A}|L\rangle_{B}\right) .
\end{aligned}
$$

Here $A$ and $B$ correspond to the up and down sides of the emission cones, respectively. By encoding the qubits 1 and 4 with $E / I$ DOF, qubits 2 and 5 with $H / V$ DOF, and qubits 3 and 6 with $R / L \mathrm{DOF}$, the desired twophoton six-qubit linear cluster state $\left|\widetilde{L C}_{6}\right\rangle$ is described as

$$
\begin{aligned}
\left|\widetilde{L C_{6}}\right\rangle= & \frac{1}{2}\left[|E E\rangle_{A B}\left|\Phi^{-}\right\rangle|L R\rangle_{A B}+|E E\rangle_{A B}\left|\Phi^{+}\right\rangle|R L\rangle_{A B}\right. \\
& \left.-|I I\rangle_{A B}\left|\Psi^{-}\right\rangle|L R\rangle_{A B}+|I I\rangle_{A B}\left|\Psi^{+}\right\rangle|R L\rangle_{A B}\right] .
\end{aligned}
$$

The transformation from the hyperentangled state to the cluster state is carried out by applying half-wave plates (HWPs) oriented at $45^{\circ}$ on the internal $A$ modes and HWPs oriented at $0^{\circ}$ on the left $B$ modes. The fidelity of the generated state is measured and $F=0.6350 \pm 0.0008$ is obtained, which is $7 \%$ better than the best previous result for six-qubit graph state with six particles. The characterization and application of this state [22] were also investigated in 2010.

Later, the hyperentangled state has been extended to ten-qubit Schrödinger cat state in experiment, which carries the genuine multi-qubit entanglement [23]. Although the previous schemes demonstrated the hyperentangled states with really high dimensions, they were only focused on two-photon states. In 2010, Gao et al. 23] generated the genuine multipartite hyperentanglement. The generation in their demonstration is composed of two steps. In the first step, a five-photon polarization entangled cat state is prepared by post-selection. Two pairs of entangled photons are produced by SPDC in the state $(|H H\rangle+|V V\rangle) / \sqrt{2}$ and a single photon is prepared in $(|H\rangle+|V\rangle) / \sqrt{2}$ state. The principle of the first step is shown in Fig. 7. Two polarizing beam splitters (PBSs), which transmit the horizontal state $|H\rangle$ and reflect the vertical state $|V\rangle$, are used to post-select the five-photon cat state $|C a t\rangle_{p}^{5}=\left(|H\rangle^{\otimes 5}+|V\rangle^{\otimes 5}\right) / \sqrt{2}$. In detail, the situation that each numbered spatial mode has one and only one photon kept, which corresponds to the desired state. Then, each photon is guided to a PBS, and the ten-qubit hyperentangled state is produced:

$$
|C a t\rangle^{10}=\frac{1}{\sqrt{2}}\left(|H\rangle^{\otimes 5}|R\rangle^{\otimes 5}+|V\rangle^{\otimes 5}|L\rangle^{\otimes 5}\right) .
$$

Here $L$ and $R$ signify the two spatial modes of each photon.

So far, the generation of entangled state is implemented via the nonlinear optical process of the SPDC in different types of nonlinear crystals. Recently, some works have been focused on waveguides due to their high efficiency and on-chip integratability. In 2014, a hyperentangled photon source in semiconductor waveguides was proposed and demonstrated, which offers an alternative path to realize an electrically pumped hyperentangled photon source [24]. They utilized phase-matching in 


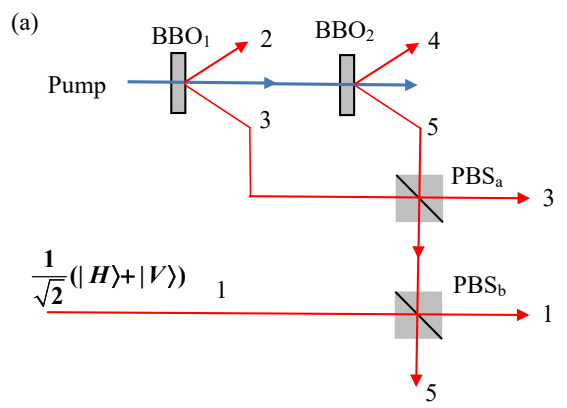

(b)

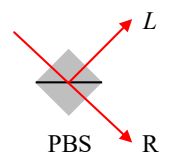

FIG. 7: Experiment setup for generating ten-qubit fivephoton hyperentangled state [23]. (a) Generation of fivephoton polarization entangled state with postselection. (b) Creation of polarization-spatial mode entanglement with PBS. $L$ and $R$ are two different spatial modes.

Bragg reflection waveguides to produce hyperentangled pairs through two type-II SPDC processes. The ideal hyperentangled state in mode and polarization DOFs is

$$
\frac{1}{2}\left(|H, V\rangle+\mathrm{e}^{\mathrm{i} \phi}|V, H\rangle\right) \otimes\left(|B, T\rangle+\mathrm{e}^{\mathrm{i} \psi}|T, B\rangle\right) .
$$

Here $T$ and $B$ denote the total internal reflection (TIR) mode and Bragg mode, respectively. The fully entangled fraction of the generated state is calculated, whose maximum value can achieve 0.99 . (a)

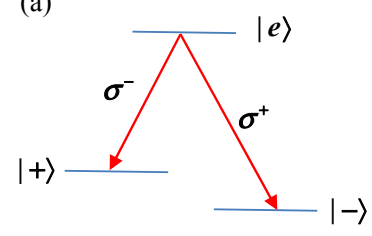

(b)

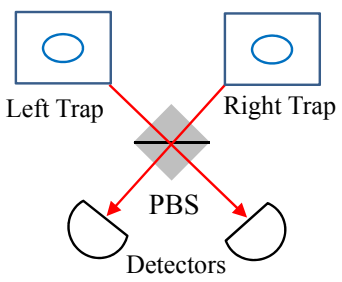

FIG. 8: Experiment principle for generating hyperentangled states between atomic ions [25]. (a) Internal energy levels of ion. The $|+\rangle$ and $|-\rangle$ are ground states and $|e\rangle$ is the excited state. $\sigma^{+}$and $\sigma^{-}$are the polarized photons emitted due to the energy decay. (b) The setup of generating spin entanglement between two atomic ions confined in separated Paul traps.

Usually, the entanglement can only be generated locally. Since the photon is one of the most ideal candidates for quantum communication, most of the previous hyperentanglement generation schemes are based on photons. Actually, other physical entity can also be used as the carrier of hyperentanglement. For example, Hu et al. [25] proposed a scheme of generating four-qubit hyperentangled state between a pair of distant non-interacting atomic ions which are confined in Paul traps. The state is entangled in both spin and motion DOFs. The atomic ions with a $\Lambda$ configuration move along one direction in the Paul trap. The principle for generating hyperentangled states between atomic ions is shown in Fig. 8. First, the ions in each trap are excited to the excited state $|e\rangle$ in spin DOF. Then they decay along two possible channels $|e\rangle \rightarrow|+\rangle$ and $|e\rangle \rightarrow|-\rangle$ accompanied by the emission of a $\sigma^{-}$(with the spin -1 ) or $\sigma^{+}$(with the spin +1 ) polarized photon, respectively. Therefore, the system consisting of the spin state of the ion and the polarization of the emitting photon is in a maximally entangled state:

$$
\left|\Psi_{1}\right\rangle=\frac{1}{\sqrt{2}}\left(|+\rangle\left|\sigma^{-}\right\rangle+|-\rangle\left|\sigma^{+}\right\rangle\right) \text {. }
$$

Then the two emitting photons from two traps are guided to a PBS. By post-selecting the case that each atom emits a single photon, the two ions are entangled in spin DOF as

$$
\left|\Psi_{2}\right\rangle=\frac{1}{\sqrt{2}}\left(|+\rangle_{L}|-\rangle_{R}+|-\rangle_{L}|+\rangle_{R}\right) .
$$

Here $L$ and $R$ denote the left and the right traps, respectively, as shown in Fig. 8b. The motion DOF of both ions is initially in the ground state $|0\rangle_{L}|0\rangle_{R}$. Then the entanglement is transferred to the motion DOF with a sequence of laser pulses.

$$
\left|\Psi_{3}\right\rangle=\frac{1}{\sqrt{2}}|+\rangle_{\mathrm{L}}|+\rangle_{\mathrm{R}}\left(|0\rangle_{\mathrm{L}}|0\rangle_{\mathrm{R}}-|1\rangle_{\mathrm{L}}|1\rangle_{\mathrm{R}}\right) .
$$

Finally, by repeating the first step, the following hyperentangled state can be produced,

$$
|\Phi\rangle=\frac{1}{2}\left(|+\rangle_{L}|-\rangle_{R}+|-\rangle_{L}|+\rangle_{R}\right) \otimes\left(|0\rangle_{L}|0\rangle_{R}-|1\rangle_{L}|1\rangle_{R}\right) .
$$

This proposal is experimentally feasible, although it has not been demonstrated in labs.

\section{HIGH-CAPACITY QUANTUM COMMUNICATION WITH HYPERENTANGLEMENT}

\section{A. Status of Bell-state analysis for photonic quantum systems}

Bell-state analysis (BSA), which is used to distinguish the four orthogonal Bell states of a two-particle quantum system in one DOF, is the prerequisite for quantum communication protocols with entanglement and it is one of the important parts in quantum repeaters. In 1999, two linear optical BSA protocols were proposed by Vaidman's 64 and Lütkenhau's 65] groups, respectively, where the success probability is $50 \%$. When hyperentanglement is used to assist the analysis of Bell states, one can completely distinguish all the four Bell states of a two-photon system in one DOF. For example, in 1998, Kwiat and Weinfurter 27] proposed two complete BSA protocols by using the hyperentanglement, which can distinguish the four orthogonal Bell states in polarization DOF with the 
success probability 100\%. In 2003, Walborn et al. [28] presented two complete BSA protocols for photon pairs entangled in one DOF with hyperentanglement, resorting to linear optical elements. Schuck et al. 29] and Barbieri et al. [30] demonstrated the complete BSA protocols in experiment by assisting hyperentanglement.

In high-capacity long-distance quantum communication, HBSA is also required to attach some important goals, especially in high-capacity quantum repeaters, teleportation of an unknown quantum state in two or more DOFs, and hyperentanglement swapping. In 2007, Wei et al. 44] proposed a HBSA protocol with linear optical elements, which can only distinguish 7 hyperentangled Bell states from 16 hyperentangled Bell states. In order to completely distinguish the 16 hyperentangled Bell states, nonlinear optical elements are required.

The complete HBSA originates from the work by Sheng et al. [36] in 2010. They proposed the first scheme for the complete HBSA of the two-photon polarizationspatial hyperentangled states with cross-Kerr nonlinearity and designed the pioneering model for teleporting an unknown quantum state in more than one DOF. In 2012, Ren et al. [37] introduced another interesting scheme for the complete HBSA for photon systems by using the giant nonlinear optics in quantum-dot-cavity systems and presented the hyperentanglement swapping with photonic polarization-spatial hyperentanglement. In 2012, Wang et al. 38] presented an important scheme for the complete HBSA for photon systems by the giant circular birefringence induced by double-sided quantum-dot-cavity systems. In 2015, Liu and Zhang 39] proposed two important schemes for hyperentangled-Bell-state generation and HBSA assisted by nitrogen-vacancy (NV) centers in resonators. Li and Ghose [41] presented a very simple scheme for the self-assisted complete maximally hyperentangled state analysis via the cross-Kerr nonlinearity and another interesting HBSA scheme [42] for polarization and time-bin hyperentanglement. Up to now, there are several important schemes for the analysis of hyperentangled states [36 45], including the probabilistic one based on linear optical elements [44] and the one for hyperentangled Greenberger-Horne-Zeilinger (GHZ) states [45]. In 2015, Wang et al. 66] demonstrated in experiment the quantum teleportation of an unknown quantum state of a single photon in multiple DOFs by implementing the HBSA of two-photon systems probabilistically with linear optical elements and ancillary entanglement sources. In 2016, Liu et al. [40] gave the original scheme for the complete nondestructive analysis of twophoton six-qubit hyperentangled Bell states assisted by cross-Kerr nonlinearity.

Here, we will introduce two high-capacity quantum communication protocols, including teleportation of an unknown quantum state of a single photon in two DOFs with hyperentanglement [36] and hyperentanglement swapping [36, 37]. First, we introduce the complete HBSA for the polarization and spatial-mode DOFs of photon systems [36], which is an important technique in high-capacity long-distance quantum communication. In the second part, we introduce the quantum teleportation protocol based on polarization-spatial hyperentanglement 36]. At last, a hyperentanglement swapping protocol [36, 37] is introduced for quantum repeater and quantum communication.

\section{B. Hyperentangled Bell-state analysis}

The polarization-spatial hyperentangled Bell state is defined as the two-photon system entangled in both the polarization and spatial-mode DOFs, such as $\left|\Phi^{+}\right\rangle_{P S}^{A B}=$ $\frac{1}{2}(|H H\rangle+|V V\rangle)_{P}^{A B} \otimes\left(\left|a_{1} b_{1}\right\rangle+\left|a_{2} b_{2}\right\rangle\right)_{S}^{A B}$. Here the superscripts $A$ and $B$ represent the two photons, and the subscripts $P$ and $S$ represent the polarization and spatialmode DOFs, respectively. $\left|i_{1}\right\rangle$ and $\left|i_{2}\right\rangle$ represent the two spatial modes of photon $i(i=A, B)$. The polarization Bell states and the spatial-mode Bell states are defined as

$$
\begin{aligned}
\left|\phi^{ \pm}\right\rangle_{P}^{A B} & =\frac{1}{\sqrt{2}}(|H H\rangle \pm|V V\rangle)_{P}^{A B}, \\
\left|\psi^{ \pm}\right\rangle_{P}^{A B} & =\frac{1}{\sqrt{2}}(|H V\rangle \pm|V H\rangle)_{P}^{A B} \\
\left|\phi^{ \pm}\right\rangle_{S}^{A B} & =\frac{1}{\sqrt{2}}\left(\left|a_{1} b_{1}\right\rangle \pm\left|a_{2} b_{2}\right\rangle\right)_{S}^{A B} \\
\left|\psi^{ \pm}\right\rangle_{S}^{A B} & =\frac{1}{\sqrt{2}}\left(\left|a_{1} b_{2}\right\rangle \pm\left|a_{2} b_{1}\right\rangle\right)_{S}^{A B}
\end{aligned}
$$

where $\left|\psi^{ \pm}\right\rangle_{P}^{A B}$ and $\left|\psi^{ \pm}\right\rangle_{S}^{A B}$ are the Bell states in the oddparity mode, and $\left|\phi^{ \pm}\right\rangle_{P}^{A B}$ and $\left|\phi^{ \pm}\right\rangle_{S}^{A B}$ are the Bell states in the even-parity mode. The 16 orthogonal hyperentangled Bell states can be distinguished completely by using polarization parity-check quantum nondemolition detectors (QNDs) and spatial-mode parity-check QNDs, assisted by cross-Kerr nonlinearity.

The Hamiltonian of cross-Kerr nonlinearity is described as $H_{\mathrm{ck}}=\hbar \chi a_{s}^{\dagger} a_{s} a_{p}^{\dagger} a_{p}$, where $\hbar \chi$ represents the coupling strength of the nonlinear material. $a_{s}^{\dagger}\left(a_{s}\right)$ and $a_{p}^{\dagger}\left(a_{p}\right)$ are the creation (annihilation) operators. With this cross-Kerr interaction, the system composed of a single photon and a coherent state can be evolved as

$$
(a|0\rangle+b|1\rangle)|\alpha\rangle \rightarrow a|0\rangle|\alpha\rangle+b|1\rangle\left|\alpha \mathrm{e}^{\mathrm{i} \theta}\right\rangle,
$$

where $|0\rangle$ and $|1\rangle$ represent the Fock states that contain 0 and 1 photon, respectively. $|\alpha\rangle$ represents a coherent state. $\theta=\chi t$ represents a phase shift with the interaction time $t$. With this cross-Kerr nonlinearity, the HBSA protocol for the 16 polarization-spatial hyperentangled Bell states can be implemented with two steps, including the spatial-mode Bell-state analysis and the polarization Bell-state analysis, shown in Figs. 9 and [10, respectively. 


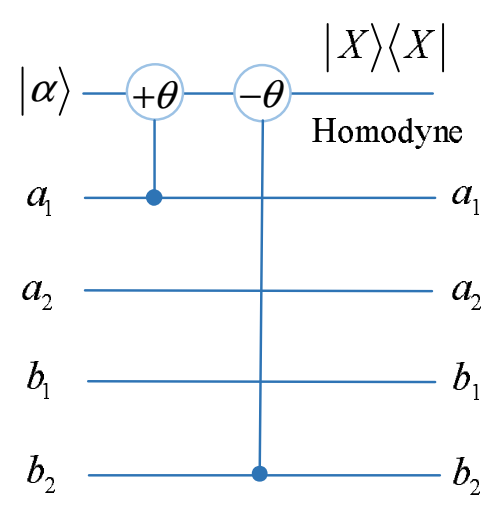

(a)

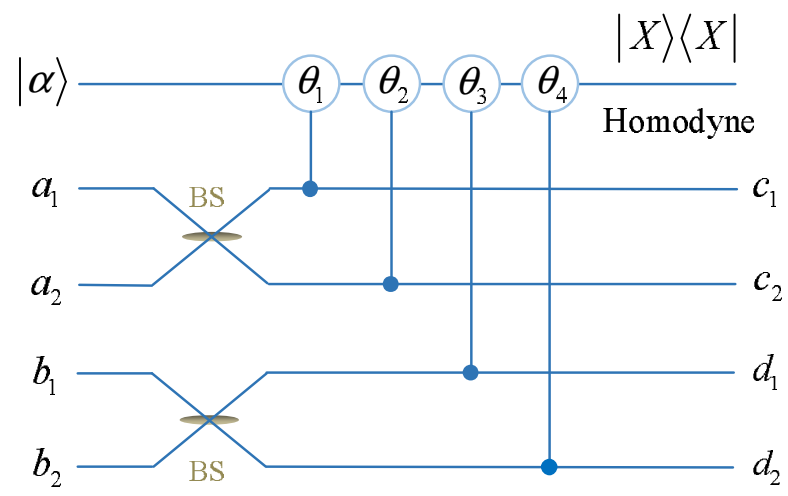

(b)

FIG. 9: Schematic diagram of the HBSA for the spatial-mode Bell states, resorting to the cross-Kerr nonlinearities [36]. (a) The spatial-mode parity-check QND for distinguishing the states $\left|\phi^{ \pm}\right\rangle_{S}^{A B}$ from $\left|\psi^{ \pm}\right\rangle_{S}^{A B}$. (b) The second step for the analysis of spatial-mode Bell states, which is used to distinguish the state $\left|\psi^{+}\right\rangle_{S}^{A B}\left(\left|\phi^{+}\right\rangle_{S}^{A B}\right)$ with the relative phase shift 0 from the state $\left|\psi^{-}\right\rangle_{S}^{A B}\left(\left|\phi^{-}\right\rangle_{S}^{A B}\right)$ with the relative phase shift $\pi$. BS represents a 50:50 beam splitter, which is used to perform a Hadamard operation on the spatial-mode DOF of a photon.

The setup of the spatial-mode Bell-state analysis is shown in Fig. 9, which is constructed with the spatialmode parity-check QNDs. After the two photons $A$ and $B$ pass through the quantum circuit shown in Fig. 9a in sequence, the state of the quantum system composed of the two-photon system $A B$ in the spatial-mode DOF and the coherent state $|\alpha\rangle$ evolves to

$$
\begin{aligned}
\left|\phi^{ \pm}\right\rangle_{S}^{A B}|\alpha\rangle & \rightarrow \frac{1}{\sqrt{2}}\left(\left|a_{1} b_{1}\right\rangle\left|\alpha \mathrm{e}^{\mathrm{i} \theta}\right\rangle \pm\left|a_{2} b_{2}\right\rangle\left|\alpha \mathrm{e}^{-\mathrm{i} \theta}\right\rangle\right), \\
\left|\psi^{ \pm}\right\rangle_{S}^{A B}|\alpha\rangle & \rightarrow \frac{1}{\sqrt{2}}\left(\left|a_{1} b_{2}\right\rangle|\alpha\rangle \pm\left|a_{2} b_{1}\right\rangle|\alpha\rangle\right) .
\end{aligned}
$$

Then the coherent beam is detected by an X-quadrature measurement, and the states $\left|\alpha \mathrm{e}^{\mathrm{i} \theta}\right\rangle$ and $\left|\alpha \mathrm{e}^{-\mathrm{i} \theta}\right\rangle$ cannot be distinguished. Hence the Bell states $\left|\phi^{ \pm}\right\rangle_{S}^{A B}$ can be distinguished from the Bell states $\left|\psi^{ \pm}\right\rangle_{S}^{A B}$ with the homodyne-heterodyne measurements. If the coherent state has a phase shift $\theta(-\theta)$, the spatial-mode state of the two-photon system $A B$ is one of the states $\left|\phi^{ \pm}\right\rangle_{S}^{A B}$. If the coherent state has no phase shift, the spatial-mode state of the two-photon system $A B$ is one of the states $\left|\psi^{ \pm}\right\rangle_{S}^{A B}$.

Subsequently, the two photons $A$ and $B$ are put into the BS shown in Fig. 9p in sequence, and the spatialmode state of the two-photon system $A B$ is transformed into

$$
\begin{aligned}
& \left|\phi^{+}\right\rangle_{S}^{A B} \rightarrow\left|\phi^{+}\right\rangle_{S}^{A B}, \quad\left|\phi^{-}\right\rangle_{S}^{A B} \rightarrow\left|\psi^{+}\right\rangle_{S}^{A B}, \\
& \left|\psi^{+}\right\rangle_{S}^{A B} \rightarrow\left|\phi^{-}\right\rangle_{S}^{A B}, \quad\left|\psi^{-}\right\rangle_{S}^{A B} \rightarrow\left|\psi^{-}\right\rangle_{S}^{A B} .
\end{aligned}
$$

After the two photons $A B$ and the coherent beam pass through the cross-Kerr medium in Fig. 9b, the four spatial-mode Bell states can be distinguished by the Xquadrature measurement on the coherent beam. If the coherent state has a phase shift $\theta_{1}+\theta_{3}, \theta_{2}+\theta_{4}, \theta_{1}+\theta_{4}$, or $\theta_{2}+\theta_{3}$, the spatial mode of the two-photon system $A B$ is $c_{1} d_{1}, c_{2} d_{2}, c_{1} d_{2}$, or $c_{2} d_{1}$, respectively. The initial spatial-mode state $\left|\phi^{+}\right\rangle_{S}^{A B}$ (or $\left|\psi^{+}\right\rangle_{S}^{A B}$ ) corresponds to the spatial mode $c_{1} d_{1}$ or $c_{2} d_{2}$, and the initial spatialmode state $\left|\phi^{-}\right\rangle_{S}^{A B}$ (or $\left|\psi^{-}\right\rangle_{S}^{A B}$ ) corresponds to the spatial mode $c_{1} d_{2}$ or $c_{2} d_{1}$.

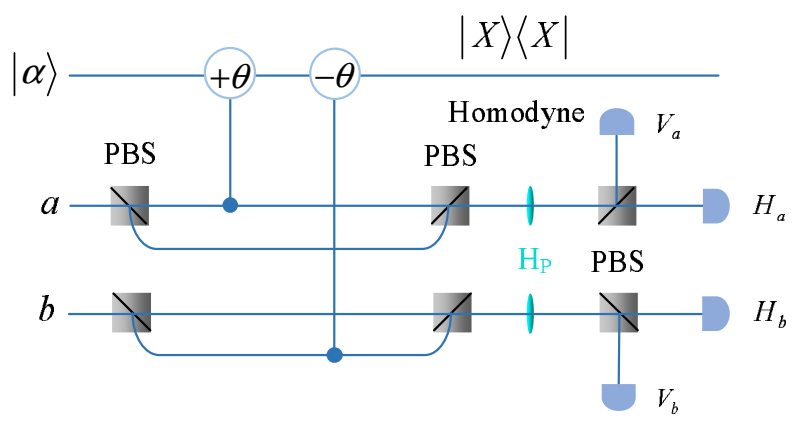

FIG. 10: Schematic diagram of the HBSA for the polarization Bell states, resorting to the cross-Kerr nonlinearities [36]. PBS represents a polarizing beam splitter, which transmits the photon in the horizontal polarization $|H\rangle$ and reflects the photon in the vertical polarization $|V\rangle$, respectively. $\mathrm{H}_{P}$ represents a half-wave plate which is used to perform a Hadamard operation on the polarization DOF of a photon. $H_{i}$ or $V_{i}$ $(i=a, b)$ represents a single-photon detector.

The setup of the polarization Bell-state analysis is shown in Fig. 10, which is constructed with the polarization parity-check QND. After the two photons $A$ and $B$ pass through the polarization parity-check QND shown in Fig. 10 in sequence, the state of the system composed of the two-photon system $A B$ in the polarization DOF 
and the coherent state $|\alpha\rangle$ evolves to

$$
\begin{aligned}
\left|\phi^{ \pm}\right\rangle_{P}^{A B}|\alpha\rangle & \rightarrow \frac{1}{\sqrt{2}}\left(|H H\rangle\left|\alpha \mathrm{e}^{\mathrm{i} \theta}\right\rangle \pm|V V\rangle\left|\alpha \mathrm{e}^{-\mathrm{i} \theta}\right\rangle\right), \\
\left|\psi^{ \pm}\right\rangle_{P}^{A B}|\alpha\rangle & \rightarrow \frac{1}{\sqrt{2}}(|H V\rangle|\alpha\rangle \pm|V H\rangle|\alpha\rangle)
\end{aligned}
$$

After the X-quadrature measurement is performed on the coherent beam, the Bell states $\left|\phi^{ \pm}\right\rangle_{P}^{A B}$ can be distinguished from the Bell states $\left|\psi^{ \pm}\right\rangle_{P}^{A B}$. If the coherent state has a phase shift $\theta(-\theta)$, the polarization state of the two-photon system $A B$ is one of the states $\left|\phi^{ \pm}\right\rangle_{P}^{A B}$. If the coherent state has no phase shift, the polarization state of the two-photon system $A B$ is one of the states $\left|\psi^{ \pm}\right\rangle_{P}^{A B}$.

Subsequently, the two photons $A$ and $B$ are put into the $\mathrm{H}_{P}$ shown in Fig. 10, and the polarization state of the two-photon system $A B$ is transformed into

$$
\begin{aligned}
\left|\phi^{+}\right\rangle_{P}^{A B} & \rightarrow\left|\phi^{+}\right\rangle_{P}^{A B}, \quad\left|\phi^{-}\right\rangle_{P}^{A B} \rightarrow\left|\psi^{+}\right\rangle_{P}^{A B}, \\
\left|\psi^{+}\right\rangle_{P}^{A B} & \rightarrow\left|\phi^{-}\right\rangle_{P}^{A B}, \quad\left|\psi^{-}\right\rangle_{P}^{A B} \rightarrow\left|\psi^{-}\right\rangle_{P}^{A B} .
\end{aligned}
$$

Then the four polarization Bell states can be distinguished by the result of four single-photon detectors. If the detectors $H_{a}, V_{b}$ (or $V_{a}, H_{b}$ ) click, the initial polarization state is $\left|\phi^{-}\right\rangle_{P}^{A B}$ (or $\left|\psi^{-}\right\rangle_{P}^{A B}$ ). If the detectors $H_{a}, H_{b}$ (or $V_{a}, V_{b}$ ) click, the initial polarization state is $\left|\phi^{+}\right\rangle_{P}^{A B}$ (or $\left|\psi^{+}\right\rangle_{P}^{A B}$ ). In this way, one can completely distinguish the 16 hyperentangled Bell states by using the spatial-mode parity-check QNDs, polarization paritycheck QND, and single-photon detectors.

\section{Teleportation with a hyperentangled channel}

The quantum teleportation protocol is used to transfer the unknown information of a quantum state between between the two remote users [67]. With hyperentanglement, two-qubit unknown information can be transferred by teleporting a photon [36].

The principle of quantum teleportation protocol with hyperentanglement is shown in Fig. 11] The photon $A$ is in the state $|\varphi\rangle_{A}=(\alpha|H\rangle+\beta|V\rangle)_{A} \otimes\left(\gamma\left|a_{1}\right\rangle+\delta\left|a_{2}\right\rangle\right)$, and the photon pair $B C$ is in a hyperentangled Bell state $\left|\phi^{+}\right\rangle_{B C}=\frac{1}{2}(|H H\rangle+|V V\rangle)_{B C} \otimes\left(\left|b_{1} c_{1}\right\rangle+\left|b_{2} c_{2}\right\rangle\right)$, where the photons $B$ and $C$ are obtained by the two remote users Alice and Bob, respectively. Alice can transfer the two-qubit information of photon $A$ to Bob by performing HBSA on the two photons $A$ and $B$.

The state of the three-photon system $A B C$ can be

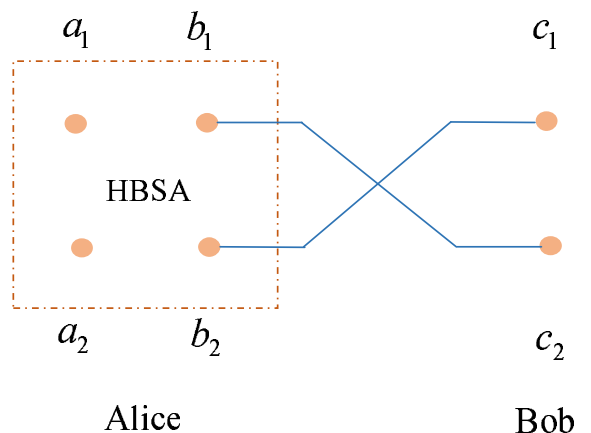

FIG. 11: Schematic diagram of the quantum teleportation protocol with hyperentanglement [36].

\section{rewritten as}

$$
\begin{aligned}
|\varphi\rangle_{A} \otimes\left|\phi^{+}\right\rangle_{B C} \\
=\frac{1}{4}\left\{\left[\left|\phi^{+}\right\rangle_{P}^{A B}(\alpha|H\rangle+\beta|V\rangle)_{c}+\left|\phi^{-}\right\rangle_{P}^{A B}(\alpha|H\rangle-\beta|V\rangle)_{c}\right.\right. \\
\left.\quad+\left|\psi^{+}\right\rangle_{P}^{A B}(\alpha|V\rangle+\beta|H\rangle)_{c}+\left|\psi^{-}\right\rangle_{P}^{A B}(\alpha|V\rangle-\beta|H\rangle)_{c}\right] \\
\quad \otimes\left[\left|\phi^{+}\right\rangle_{S}^{A B}\left(\gamma\left|c_{1}\right\rangle+\delta\left|c_{2}\right\rangle\right)+\left|\phi^{-}\right\rangle_{S}^{A B}\left(\gamma\left|c_{1}\right\rangle-\delta\left|c_{2}\right\rangle\right)\right. \\
\left.\left.\quad+\left|\psi^{+}\right\rangle_{S}^{A B}\left(\gamma\left|c_{2}\right\rangle+\delta\left|c_{1}\right\rangle\right)+\left|\psi^{-}\right\rangle_{S}^{A B}\left(\gamma\left|c_{2}\right\rangle-\delta\left|c_{1}\right\rangle\right)\right]\right\} .
\end{aligned}
$$

After Alice performs HBSA on the two photons $A$ and $B$, photon $C$ will be projected to a single-photon quantum state in two DOFs. If the outcome of HBSA for the photon pair $A B$ is $\left|\phi^{ \pm}\right\rangle_{P}\left|\phi^{ \pm}\right\rangle_{S},\left|\psi^{ \pm}\right\rangle_{P}\left|\phi^{ \pm}\right\rangle_{S},\left|\phi^{ \pm}\right\rangle_{P}\left|\psi^{ \pm}\right\rangle_{S}$, or $\left|\psi^{ \pm}\right\rangle_{P}\left|\psi^{ \pm}\right\rangle_{S}$, the state of photon $C$ is projected to $(\alpha|H\rangle \pm \beta|V\rangle)\left(\gamma\left|c_{1}\right\rangle \pm \delta\left|c_{2}\right\rangle\right),(\alpha|V\rangle \pm \beta|H\rangle)\left(\gamma\left|c_{1}\right\rangle \pm \delta\left|c_{2}\right\rangle\right)$, $(\alpha|H\rangle \pm \beta|V\rangle)\left(\gamma\left|c_{2}\right\rangle \pm \delta\left|c_{1}\right\rangle\right)$, or $(\alpha|V\rangle \pm \beta|H\rangle)\left(\gamma\left|c_{2}\right\rangle \pm\right.$ $\left.\delta\left|c_{1}\right\rangle\right)$, respectively. If the polarization (spatial-mode) state of photon pair $A B$ is $\left|\phi^{-}\right\rangle_{P}\left(\left|\phi^{-}\right\rangle_{S}\right)$, Bob should perform a polarization (spatial-mode) phase-flip operation $\sigma_{z}^{P}\left(\sigma_{z}^{S}\right)$ on photon $C$ after Alice publishes the result of HBSA. If the polarization (spatial-mode) state of photon pair $A B$ is $\left|\psi^{+}\right\rangle_{P}\left(\left|\psi^{+}\right\rangle_{S}\right)$, Bob should perform a polarization (spatial-mode) bit-flip operation $\sigma_{x}^{P}$ $\left(\sigma_{x}^{S}\right)$ on photon $C$. If the polarization (spatial-mode) state of photon pair $A B$ is $\left|\psi^{-}\right\rangle_{P}\left(\left|\psi^{-}\right\rangle_{S}\right)$, Bob should perform a unitary operation $-\mathrm{i} \sigma_{y}^{P}\left(-\mathrm{i} \sigma_{y}^{S}\right)$ on photon $C$. Then, Bob can obtain the unknown single-photon state $(\alpha|H\rangle+\beta|V\rangle)\left(\gamma\left|c_{1}\right\rangle+\delta\left|c_{2}\right\rangle\right)$. Here, $\sigma_{z}^{P}=|H\rangle\langle H|-| V\rangle\langle V|$, $\sigma_{x}^{P}=|H\rangle\langle V|+| V\rangle\left\langle H\left|, \quad-\mathrm{i} \sigma_{y}^{P}=\right| H\right\rangle\langle V|-| V\rangle\langle H|$, $\sigma_{z}^{S}=\left|c_{1}\right\rangle\left\langle c_{1}|-| c_{2}\right\rangle\left\langle c_{2}\left|, \sigma_{x}^{S}=\right| c_{1}\right\rangle\left\langle c_{2}|+| c_{2}\right\rangle\left\langle c_{1}\right|$, and $-\mathrm{i} \sigma_{y}^{S}=\left|c_{1}\right\rangle\left\langle c_{2}|-| c_{2}\right\rangle\left\langle c_{1}\right|$.

\section{Hyperentanglement swapping}

Entanglement swapping is used to obtain the entanglement between two particles that have no interaction initially, and it has been widely applied in quantum repeaters and quantum communication protocols. The 
principle of hyperentanglement swapping is shown in Fig. 12. The photon pairs $A B$ and $C D$ are initially in the hyperentangled Bell states $\left|\phi^{+}\right\rangle_{P S}^{A B}$ and $\left|\phi^{+}\right\rangle_{P S}^{C D}$, respectively. Here,

$$
\begin{aligned}
\left|\phi^{+}\right\rangle_{P S}^{A B} & =\frac{1}{2}(|H H\rangle+|V V\rangle)_{P}^{A B} \otimes\left(\left|a_{1} b_{1}\right\rangle+\left|a_{2} b_{2}\right\rangle\right)_{S}^{A B}, \\
\left|\phi^{+}\right\rangle_{P S}^{C D} & =\frac{1}{2}(|H H\rangle+|V V\rangle)_{P}^{C D} \otimes\left(\left|c_{1} d_{1}\right\rangle+\left|c_{2} d_{2}\right\rangle\right)_{S}^{C D} .
\end{aligned}
$$

The photons $B$ and $C$ belong to Alice. The photons $A$ and $D$ belong to Bob and Charlie, respectively. That is, Alice shares a hyperentangled photon pair with Bob, and she also shares a hyperentangled photon pair with Charlie. The task of hyperentanglement swapping is to obtain the hyperentangled Bell state $\left|\phi^{+}\right\rangle_{P S}^{A D}=\frac{1}{2}(|H H\rangle+$ $|V V\rangle)_{P}^{A D} \otimes\left(\left|a_{1} d_{1}\right\rangle+\left|a_{2} d_{2}\right\rangle\right)_{S}^{A D}$, which can be implemented by performing HBSA on photon pair $B C$.

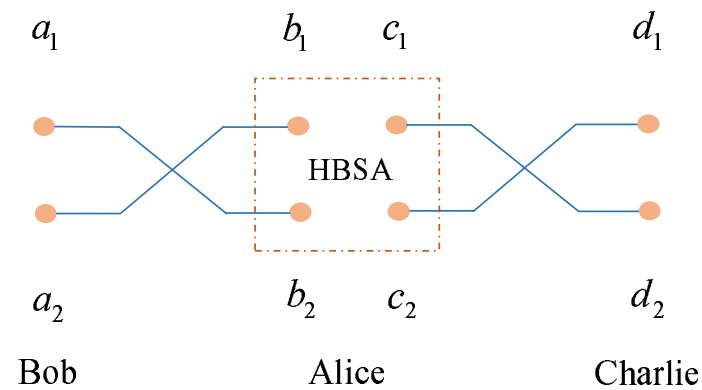

FIG. 12: Schematic diagram of the hyperentanglement swapping protocol [36, 37].

The state of the four-photon system $A B C D$ can be rewritten as

$$
\begin{aligned}
\left|\phi^{+}\right\rangle_{P S}^{A B} \otimes & \left|\phi^{+}\right\rangle_{P S}^{C D} \\
= & \frac{1}{4}\left[\left(\left|\phi^{+}\right\rangle_{P}^{A D}\left|\phi^{+}\right\rangle_{P}^{B C}+\left|\phi^{-}\right\rangle_{P}^{A D}\left|\phi^{-}\right\rangle_{P}^{B C}\right.\right. \\
& \left.+\left|\psi^{+}\right\rangle_{P}^{A D}\left|\psi^{+}\right\rangle_{P}^{B C}+\left|\psi^{-}\right\rangle_{P}^{A D}\left|\psi^{-}\right\rangle_{P}^{B C}\right) \\
& \otimes\left(\left|\phi^{+}\right\rangle_{S}^{A D}\left|\phi^{+}\right\rangle_{S}^{B C}+\left|\phi^{-}\right\rangle_{S}^{A D}\left|\phi^{-}\right\rangle_{S}^{B C}\right. \\
& \left.\left.+\left|\psi^{+}\right\rangle_{S}^{A D}\left|\psi^{+}\right\rangle_{S}^{B C}+\left|\psi^{-}\right\rangle_{S}^{A D}\left|\psi^{-}\right\rangle_{S}^{B C}\right)\right] .
\end{aligned}
$$

After Alice performs HBSA on the photon pair $B C$, the correlation between the two photons $A D$ can be created. If the outcome of HBSA for the photon pair $B C$ is $\left|\phi^{ \pm}\right\rangle_{P}\left|\phi^{ \pm}\right\rangle_{S},\left|\psi^{ \pm}\right\rangle_{P}\left|\phi^{ \pm}\right\rangle_{S},\left|\phi^{ \pm}\right\rangle_{P}\left|\psi^{ \pm}\right\rangle_{S}$, or $\left|\psi^{ \pm}\right\rangle_{P}\left|\psi^{ \pm}\right\rangle_{S}$, the state of the photon pair $A D$ is projected to $\left|\phi^{ \pm}\right\rangle_{P}\left|\phi^{ \pm}\right\rangle_{S}, \quad\left|\psi^{ \pm}\right\rangle_{P}\left|\phi^{ \pm}\right\rangle_{S}, \quad\left|\phi^{ \pm}\right\rangle_{P}\left|\psi^{ \pm}\right\rangle_{S}$, or $\left|\psi^{ \pm}\right\rangle_{P}\left|\psi^{ \pm}\right\rangle_{S}$, respectively. If the polarization (spatialmode) state of the photon pair $B C$ is $\left|\phi^{-}\right\rangle_{P}\left(\left|\phi^{-}\right\rangle_{S}\right)$, Bob should perform a unitary operation $\sigma_{z}^{P}\left(\sigma_{z}^{S}\right)$ on photon $A$ after Alice publishes the result of HBSA. If the polarization (spatial-mode) state of the photon pair $B C$ is
$\left|\psi^{+}\right\rangle_{P}\left(\left|\psi^{+}\right\rangle_{S}\right)$, Bob should perform a unitary operation $\sigma_{x}^{P}\left(\sigma_{x}^{S}\right)$ on photon $A$. If the polarization (spatial-mode) state of the photon pair $B C$ is $\left|\psi^{-}\right\rangle_{P}\left(\left|\psi^{-}\right\rangle_{S}\right)$, Bob should perform a unitary operation $-\mathrm{i} \sigma_{y}^{P}\left(-\mathrm{i} \sigma_{y}^{S}\right)$ on photon $A$. Now, Bob and Charlie can share a hyperentangled photon pair $A D$ in the state $\left|\phi^{+}\right\rangle_{P S}^{A D}$.

\section{HYPERENTANGLEMENT CONCENTRATION}

\section{A. Development of entanglement concentration}

In the practical quantum communication with entanglement, the entangled photon systems are produced locally, which leads to their decoherence when the photons are transmitted over a quantum channel with environment noise or stored in practical quantum devices. Quantum repeater is a necessary technique for long-distance quantum communication and it is used to overcome the influence from this decoherence [4]. In fact, the optimal way to overcome the influence on photon systems from channel noise in quantum communication is the selferror-rejecting qubit transmission [63] with linear optics as it is an active way to decrease the influence from channel noise and it is very efficient and simple to be implemented in experiment with current feasible techniques. However, this scheme [63] can only depress most of the influence from the channel noise in the process of photon distribution, as the same as the other active methods for overcoming the influence from noise 68 -70]. It does not work in depressing the influence of noise from both a long-distance channel and the storage process for quantum states. Moreover, quantum repeaters for longdistance quantum communication require the entangled photons with higher fidelity (usually $\sim 99 \%$ ) beyond that from faithful qubit transmission schemes (about 90\% $96 \%$ for a polarization quantum state of photons over an optical-fiber channel with several kilometers). That is, entanglement concentration and entanglement purification are not only useful but also absolutely necessary in long-distance quantum communication.

Entanglement concentration is used to distill some nonlocal entangled systems in a maximally entangled state from a set of nonlocal entangled systems in a partially entangled pure state 71]. Before 2013, entanglement concentration is focused on the nonlocal quantum states in one DOF, such as the polarization states of photons, the two-level quantum states of atom systems, or the spins of electron systems. The first entanglement concentration protocol (ECP) was proposed by Bennett et al. 71] in 1996, which is based on the Schmidt projection [71]. Also, it is just a mathematic method for entanglement concentration. In 2001, two ECPs were proposed [72, 73] with PBSs for two ideal entangled photon sources. In 2008, Sheng et al. 74] proposed a repeatable ECP to concentrate both bipartite and multipartite quantum systems, and it has an advantage of far higher 
efficiency and yield than those in Bennett's ECP [71] and the PBS-based ECPs [2, 73], by iteration of the concentration process two or three times. In fact, depending on whether the parameters of the nonlocal less-entangled states are unknown 71 75] or known [76 78], the existing ECPs can be classed into two groups. When the parameters are known, one nonlocal photon system is enough for concentrating the nonlocal entanglement efficiently [76 78] with far higher yield than those with unknown parameters 71 74]. In 1999, Bose et al. 76] designed the first ECP for nonlocal entangled photon pairs in the less-entangled pure state with known parameters, resorting to the entanglement swapping of a nonlocal entangled photon pair and a local entangled photon pair. In 2000, Shi et al. 77. proposed another ECP based on entanglement swapping and a collective unitary operation on two qubits. In 2012, Sheng et al. [78] presented two ECPs for photon systems in the less-entangled states with known parameters, according to which an ancillary single photon state can be prepared to assist the concentration. In 2012, Deng 79] presented the optimal nonlocal multipartite ECP based on projection measurement. Also, some schemes for concentrating $\mathrm{W}$ states have been proposed [80 82]. Moreover, two groups [83, 84] demonstrated in experiment the entanglement concentration of twophoton systems with linear optical elements. A review about entanglement concentration of photon systems in one DOF with cross-Kerr nonlinearity was presented in Ref. 85].

The investigation on hyperentanglement concentration began from 2013. In this year, Ren et al. [46] proposed the parameter-splitting method to extract the maximally entangled photons in both the polarization and spatial-mode DOFs when the coefficients of the initial partially hyperentangled states are known. This fascinating (novel) method is very efficient and simple in terms of concentrating partially entangled states as it can be achieved with the maximal success probability by performing the protocol only once, resorting to linear-optical elements only, not nonlinearity, no matter what the form of the known nonlocal entangled state is, what the number of the DOFs is, and what the number of particles in the quantum system is. They [46] also gave the first hyperentanglement concentration protocol (hyper-ECP) for the unknown polarization-spatial lesshyperentangled states with linear-optical elements only and another hyper-ECP [47] for nonlocal polarizationspatial less-hyperentangled states with unknown parameters assisted by diamond nitrogen vacancy (NV) centers inside photonic crystal cavities. Subsequently, Ren and Long [48] proposed a general hyper-ECP for photon systems assisted by quantum-dot spins inside optical microcavities and another high-efficiency hyper-ECP [49] with the quantum-state-joining method. In the same time, Li and Ghose [50] presented a hyper-ECP resorting to linear optics. In 2015, they also brought forward an efficient hyper-ECP for the multipartite hyperentangled state via the cross-Kerr nonlinearity [51] and another
hyper-ECP for time-bin and polarization hyperentangled photons [52]. In 2016, Cao et al. 53] presented a hyperECP for entangled photons by using photonic module system.

In this section, we overview the hyper-ECPs for highcapacity long-distance quantum communication [46], resorting to the parameter-splitting method [46] and the Schmidt projection method 71, respectively. With the parameter-splitting method [4], the hyper-ECP can be implemented with the maximal success probability [46], resorting to linear optical elements only. With the Schmidt projection method, the success probability of the hyper-ECP is relatively low with linear optical elements [46, 50, 52], and it can be improved by iterative application of the hyper-ECP process with nonlinear optical elements [47, 48].

\section{B. Hyper-ECP with parameter-splitting method}

The parameter-splitting method is introduced to concentrate nonlocal partially entangled states with their parameters accurately known to the remote users [46]. With this method, only one remote user has to perform local operations with linear optical elements, and the success probability of the ECP can achieve the maximal value. The ECP for polarization (spatial-mode) DOF of photon system is introduced in detail in Ref. [46]. Here, we introduce the hyper-ECP for polarization-spatial hyperentangled Bell state by using the parameter-splitting method [46]. That is, Alice and Bob obtain a subset of nonlocal two-photon systems in a maximally hyperentangled Bell state by splitting the parameters of the partially hyperentangled Bell states with linear optical elements only.

The partially hyperentangled Bell state is described as

$\left|\phi_{0}\right\rangle_{A B}=(\alpha|H H\rangle+\beta|V V\rangle)_{A B} \otimes\left(\gamma\left|a_{1} b_{1}\right\rangle+\delta\left|a_{2} b_{2}\right\rangle\right)$,

where the subscripts $A$ and $B$ represent two photons obtained by the two remote users, Alice and Bob, respectively. $\alpha, \beta, \gamma$, and $\delta$ are four real parameters that are known to the two remote users, and they satisfy the relation $|\alpha|^{2}+|\beta|^{2}=|\gamma|^{2}+|\delta|^{2}=1$.

The setup of the hyper-ECP [46] for the partially hyperentangled Bell state $\left|\phi_{0}\right\rangle_{A B}$ is shown in Fig. 13b. It is implemented by performing some local unitary operations on both the spatial-mode and polarization DOFs of photon $A$. No operation is performed on photon $B$. To describe the principle of the hyper-ECP explicitly and simply, the four parameters are chosen as $|\alpha|>|\beta|$ and $|\gamma|<|\delta|$. In other cases, the hyper-ECP can be implemented as the same as this one with or without a little modification.

First, Alice splits the parameter of the spatial-mode state by performing a unitary operation on spatial mode 


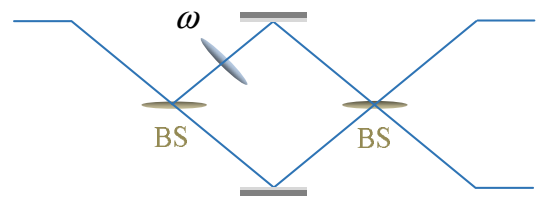

(a)
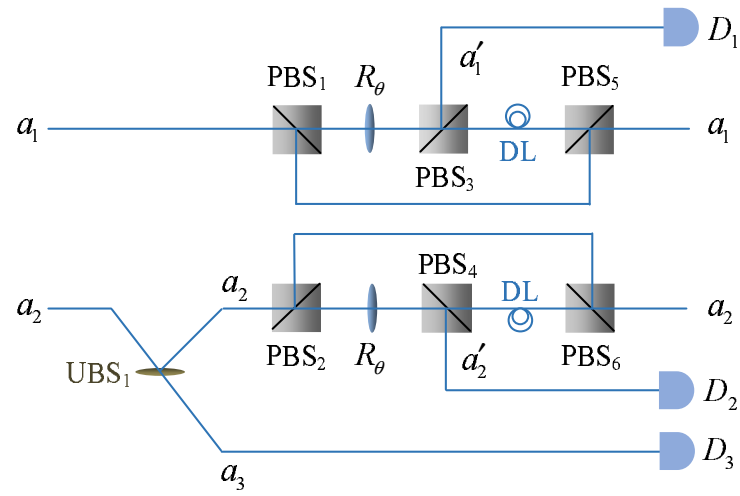

(b)

FIG. 13: (a) Schematic diagram of an unbalanced BS (UBS) 46]. $\omega$ represents a wave plate which can cause a phase shift between the two spatial modes. (b) Schematic diagram of the polarization-spatial hyper-ECP with the parameter-splitting method [46]. UBS represents an unbalanced beam splitter with the reflection coefficient $R=\gamma / \delta$. $R_{\theta}$ represents a wave plate which is used to rotate the horizontal polarization with an angle $\theta=\arccos (\beta / \alpha)$. DL denotes a time-delay device which is used to make the two wavepackets of the two spatial modes arrive at $\mathrm{PBS}_{5}\left(\right.$ or $\left.\mathrm{PBS}_{6}\right)$ in the same time. $D_{i}(i=$ $1,2,3)$ represents a single-photon detector.

$a_{2}$, resorting to an unbalanced beam splitter (i.e., UBS) with reflection coefficient $R=\gamma / \delta$ (shown in Fig. 13a). The state of the photon pair $A B$ is changed from $\left|\phi_{0}\right\rangle_{A B}$ to $\left|\phi_{1}\right\rangle_{A B}$. Here

$$
\begin{aligned}
\left|\phi_{1}\right\rangle_{A B}= & (\alpha|H H\rangle+\beta|V V\rangle)_{A B} \otimes\left[\gamma\left(\left|a_{1} b_{1}\right\rangle+\left|a_{2} b_{2}\right\rangle\right)\right. \\
& \left.+\sqrt{|\delta|^{2}-|\gamma|^{2}}\left|a_{3} b_{2}\right\rangle\right]
\end{aligned}
$$

If photon $A$ is not detected in the spatial mode $a_{3}$, the spatial-mode state of the photon pair $A B$ is transformed into a maximally entangled Bell state.

Subsequently, Alice splits the parameter of the polarization state by performing the same polarization unitary operations on the spatial modes $a_{1}$ and $a_{2}$ as shown in Fig. 13b. After two spatial modes $a_{1}$ and $a_{2}$ pass through PBSs (i.e., $\mathrm{PBS}_{1}$ and $\mathrm{PBS}_{2}$ ) and $R_{\theta}$, the state of the photon pair $A B$ is transformed from $\left|\phi_{1}\right\rangle_{A B}$ to $\left|\phi_{2}\right\rangle_{A B}$. Here

$$
\begin{aligned}
\left|\phi_{2}\right\rangle_{A B}= & {\left[\beta(|H H\rangle+|V V\rangle)+\sqrt{|\alpha|^{2}-|\beta|^{2}}\left|V^{\prime} H\right\rangle\right]_{A B} } \\
& \otimes \gamma\left(\left|a_{1} b_{1}\right\rangle+\left|a_{2} b_{2}\right\rangle\right)+(\alpha|H H\rangle+\beta|V V\rangle)_{A B} \\
& \otimes \sqrt{|\delta|^{2}-|\gamma|^{2}}\left|a_{3} b_{2}\right\rangle,
\end{aligned}
$$

where $\left|V^{\prime}\right\rangle$ represents the vertical polarization of photon $A$ after an operation $R_{\theta}$. The wave plate $R_{\theta}$ is used to perform a rotate operation $|H\rangle \rightarrow \cos \theta|H\rangle+\sin \theta|V\rangle$ on the horizontal polarization $|H\rangle$.

Finally, Alice lets two spatial modes $a_{1}$ and $a_{2}$ pass through $\mathrm{PBS}_{3}, \mathrm{PBS}_{4}, \mathrm{DL}, \mathrm{PBS}_{5}$ and $\mathrm{PBS}_{6}$, and the state of the photon pair $A B$ is transformed from $\left|\phi_{2}\right\rangle_{A B}$ to $\left|\phi_{3}\right\rangle_{A B}$. Here

$$
\begin{aligned}
\left|\phi_{3}\right\rangle_{A B}= & \beta \gamma(|H H\rangle+|V V\rangle)_{A B}\left(\left|a_{1} b_{1}\right\rangle+\left|a_{2} b_{2}\right\rangle\right) \\
& +\gamma \sqrt{|\alpha|^{2}-|\beta|^{2}}|V H\rangle_{A B}\left(\left|a_{1}^{\prime} b_{1}\right\rangle+\left|a_{2}^{\prime} b_{2}\right\rangle\right) \\
& +\sqrt{|\delta|^{2}-|\gamma|^{2}}(\alpha|H H\rangle+\beta|V V\rangle)_{A B}\left|a_{3} b_{2}\right\rangle .
\end{aligned}
$$

If photon $A$ is not detected in one of the spatial modes $a_{1}^{\prime}$ and $a_{2}^{\prime}$, the polarization state of the photon pair $A B$ is transformed into a maximally entangled Bell state. That is, the maximally hyperentangled Bell state $|\phi\rangle_{A B}$ is obtained. Here

$$
|\phi\rangle_{A B}=\frac{1}{2}(|H H\rangle+|V V\rangle)_{A B}\left(\left|a_{1} b_{1}\right\rangle+\left|a_{2} b_{2}\right\rangle\right) .
$$

If photon $A$ is detected in one of the spatial modes $a_{1}^{\prime}$, $a_{2}^{\prime}$, and $a_{3}$, the polarization DOF or the spatial-mode DOF of the photon pair $A B$ will project to a product state, which means the hyper-ECP fails. According to the detection in the spatial modes of photon $A$, Alice can read out whether the hyper-ECP succeeds or not in theory. As the efficiency of a single-photon detector is lower than $100 \%$, the mistaken of a successful event caused by the detection inefficiency can be eliminated by postselection.

The success probability of this hyper-ECP is $P=$ $4|\beta \gamma|^{2}$, which achieves the maximal success probability for obtaining a maximally hyperentangled Bell state from a partially hyperentangled Bell state. Moreover, this parameter-splitting method is suitable for all the entanglement concentration of photon systems in nonlocal partially entangled pure states with known parameters, including those based on one DOF and those based on multiple DOFs.

\section{Hyper-ECP with Schmidt projection method}

Here, we mainly introduce two hyper-ECPs for polarization-spatial hyperentangled Bell states with unknown parameters [46]. The first one is implemented with linear optical elements [46], which is much easier to achieve in experiment. The second one is implemented with nonlinear optical elements [47], which can improve the success probability by iterative application of the hyper-ECP.

\section{Hyper-ECP with linear optical elements}

In the Schmidt projection method, two identical photon pairs $A B$ and $C D$ are required, which are in the 
partially hyperentangled Bell states $\left|\phi_{0}\right\rangle_{A B}$ and $\left|\phi_{0}\right\rangle_{C D}$, respectively. Here

$$
\begin{aligned}
& \left|\phi_{0}\right\rangle_{A B}=(\alpha|H H\rangle+\beta|V V\rangle)_{A B} \otimes\left(\gamma\left|a_{1} b_{1}\right\rangle+\delta\left|a_{2} b_{2}\right\rangle\right), \\
& \left|\phi_{0}\right\rangle_{C D}=(\alpha|H H\rangle+\beta|V V\rangle)_{C D} \otimes\left(\gamma\left|c_{1} d_{1}\right\rangle+\delta\left|c_{2} d_{2}\right\rangle\right) .
\end{aligned}
$$

Here the subscripts $A B$ and $C D$ represent two photon pairs shared by the two remote users. Alice has the two photons $A$ and $C$, and Bob has the two photons $B$ and $D$. $\alpha, \beta, \gamma$, and $\delta$ are four unknown real parameters, and they satisfy the relation $|\alpha|^{2}+|\beta|^{2}=|\gamma|^{2}+|\delta|^{2}=1$.

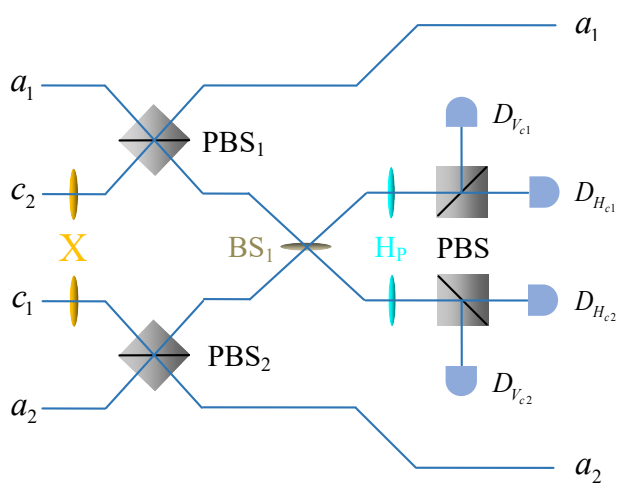

(a)

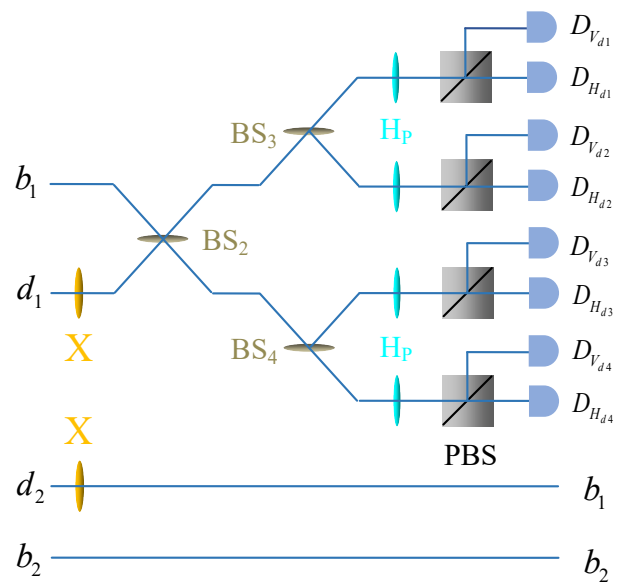

(b)

FIG. 14: Schematic diagram of the polarization-spatial hyperECP for partially hyperentangled Bell states with unknown parameters [46], resorting to the Schmidt projection method. (a) Operations performed by Alice. (b) Operations performed by Bob. X represents a half-wave plate which is used to perform a polarization bit-flip operation $\sigma_{x}^{P}=|H\rangle\langle V|+| V\rangle\langle H|$.

The setup of the hyper-ECP with the Schmidt projection method for unknown partially hyperentangled Bell states [46] is shown in Fig. 14. The initial state of four-photon system $A B C D$ is described as: $\left|\Phi_{0}\right\rangle=$ $\left|\phi_{0}\right\rangle_{A B} \otimes\left|\phi_{0}\right\rangle_{C D}$. After bit-flip operations $\left(\sigma_{x}^{P}\right)$ are performed on the polarization DOF of photons $C$ and $D$, the state of four-photon system $A B C D$ becomes

$$
\begin{aligned}
\left|\Phi_{1}\right\rangle= & \left(\alpha^{2}|H H V V\rangle+\alpha \beta|V V V V\rangle+\alpha \beta|H H H H\rangle\right. \\
& \left.+\beta^{2}|V V H H\rangle\right)_{A B C D} \otimes\left(\gamma^{2}\left|a_{1} b_{1} c_{1} d_{1}\right\rangle\right. \\
& \left.+\gamma \delta\left|a_{2} b_{2} c_{1} d_{1}\right\rangle+\gamma \delta\left|a_{1} b_{1} c_{2} d_{2}\right\rangle+\delta^{2}\left|a_{2} b_{2} c_{2} d_{2}\right\rangle\right) .
\end{aligned}
$$

Subsequently, the wavepackets from the spatial modes $a_{1}$ and $c_{2}$ are put into $\mathrm{PBS}_{1}$, and the wavepackets from the spatial modes $a_{2}$ and $c_{1}$ are put into $\mathrm{PBS}_{2}$. The wavepackets from the spatial modes $b_{1}$ and $d_{1}$ are put into $\mathrm{BS}_{2}$. Here PBSs in Fig. 14a are used to perform a polarization parity-check measurement on the two photons $A$ and $C$, and BS in Fig. 14b is used to perform a spatial-mode parity-check measurement on the two photons $B$ and $D$ with the Hong-Ou-Mandel (HOM) effect [86]. If the photon pair $A C$ is in an even-parity polarization mode (i.e. $|H H\rangle_{A C}$ and $|V V\rangle_{A C}$ ), only one photon will be detected by Alice in principle, as shown in Fig. 14a. If the photon pair $A C$ is in an odd-parity polarization mode (i.e. $|H V\rangle_{A C}$ and $|V H\rangle_{A C}$ ), both of the two photons $A$ and $C$ will be detected or undetected in principle. If the photon pair $B D$ is in an odd-parity spatial mode (i.e. $\left|b_{1} d_{2}\right\rangle$ and $\left|b_{2} d_{1}\right\rangle$ ), only one photon will be detected by Bob in principle as shown in Fig. 14 $\mathrm{b}$. If the photon pair $B D$ is in an even-parity spatial mode (i.e. $\left|b_{1} d_{1}\right\rangle$ and $\left.\left|b_{2} d_{2}\right\rangle\right)$, both of the two photons $B$ and $D$ will be detected or undetected in principle.

With the polarization parity-check measurement and spatial-mode parity-check measurement, Alice and Bob can divide the polarization states and the spatial-mode states of the four-photon systems into two groups, respectively. They pick up the even-parity polarization states of the photon pair $A C$ and the odd-parity spatial-mode states of the photon pair $B D$, which leads to the fact that both Alice and Bob have only one detector clicked. In this time, the state of the four-photon system $A B C D$ is projected into the state $\left|\Phi_{2}\right\rangle$. Here

$$
\begin{aligned}
\left|\Phi_{2}\right\rangle= & \frac{1}{2}(|V V V V\rangle+|H H H H\rangle)_{A B C D} \\
& \otimes\left(\left|a_{2} b_{2} c_{1} d_{1}\right\rangle+\left|a_{1} b_{1} c_{2} d_{2}\right\rangle\right) .
\end{aligned}
$$

If the outcome of the detectors is in another condition, this hyper-ECP fails.

Finally, the Hadamard operations are performed on the spatial-mode and polarization DOFs of the photons $C$ and $D$, respectively, and the state of the four-photon system $A B C D$ is transformed from $\left|\Phi_{2}\right\rangle$ to $\left|\Phi_{3}\right\rangle$. Here

$$
\begin{aligned}
\left|\Phi_{3}\right\rangle= & \frac{1}{8}\left[(|V V\rangle+|H H\rangle)_{A B}(|V V\rangle+|H H\rangle)_{C D}\right. \\
& \left.+(|H H\rangle-|V V\rangle)_{A B}(|H V\rangle+|V H\rangle)_{C D}\right] \\
& \otimes\left[\left(\left|a_{2} b_{2}\right\rangle+\left|a_{1} b_{1}\right\rangle\right)\left(\left|c_{1} d_{1}\right\rangle+\left|c_{2} d_{2}\right\rangle\right)\right. \\
& \left.-\left(\left|a_{1} b_{1}\right\rangle-\left|a_{2} b_{2}\right\rangle\right)\left(\left|c_{1} d_{2}\right\rangle+\left|c_{2} d_{1}\right\rangle\right)\right] .
\end{aligned}
$$


If the outcome of the two clicked detectors is in an even-parity polarization mode and an even-parity spatial mode, the state of the photon pair $A B$ is projected to the maximally hyperentangled Bell state $|\phi\rangle_{A B}=$ $\frac{1}{2}(|H H\rangle+|V V\rangle)_{A B}\left(\left|a_{1} b_{1}\right\rangle+\left|a_{2} b_{2}\right\rangle\right)$. If the outcome of the two clicked detectors is in an odd-parity polarization (spatial) mode, a phase-flip operation $\sigma_{z}^{P}\left(\sigma_{z}^{S}\right)$ on photon $B$ is required to obtain the state $|\phi\rangle_{A B}$.

In principle, if Alice and Bob both have only one detector clicked, the maximally hyperentangled Bell states can be obtained with the probability of $P=4|\alpha \beta \gamma \delta|^{2}$. Otherwise, the hyper-ECP fails. In practical, whether this hyper-ECP succeeds or not can also be read out by postselection, if the efficiencies of the single-photon detectors are lower than $100 \%$. With this method, the maximally hyperentangled GHZ states can be obtained from the partially hyperentangled GHZ states [50] in the same way.

\section{Hyper-ECP with nonlinear optical elements}

Here, we introduce the hyper-ECP with nonlinear optical elements. That is, the polarization parity-check measurement and the spatial-mode parity-check measurement are replaced by the polarization parity-check QND (P-QND) and the spatial-mode parity-check QND (S-QND), respectively, where the P-QND and S-QND are constructed with the cross-Kerr nonlinearity.

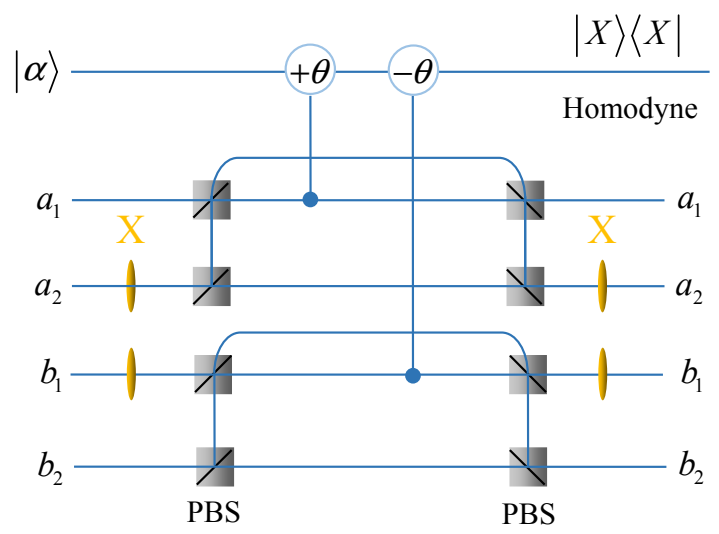

FIG. 15: Schematic diagram of the polarization parity-check quantum nondemolition detector (P-QND) with the crossKerr nonlinearity [51].

$P-Q N D$ - The setup of the P-QND is shown in Fig. 15. which is different from the one in Fig. 10, In Fig. 10, the spatial-mode state is detected at the end of the spatialmode Bell-state analysis. Here, the P-QND is required to distinguish the even-parity polarization modes from the odd-parity polarization modes without affecting the spatial-mode states. That is,

$$
\begin{aligned}
& (\alpha|H H\rangle \pm \beta|V V\rangle) \otimes\left(\gamma\left|a_{1} b_{1}\right\rangle \pm \delta\left|a_{2} b_{2}\right\rangle\right)_{A B}|\alpha\rangle \\
& \rightarrow\left(\alpha|H H\rangle\left|\alpha \mathrm{e}^{\mathrm{i} \theta}\right\rangle \pm \beta|V V\rangle\left|\alpha \mathrm{e}^{-\mathrm{i} \theta}\right\rangle\right)_{A B} \\
& \quad \otimes\left(\gamma\left|a_{1} b_{1}\right\rangle \pm \delta\left|a_{2} b_{2}\right\rangle\right), \\
& (\alpha|H H\rangle \pm \beta|V V\rangle) \otimes\left(\gamma\left|a_{1} b_{2}\right\rangle \pm \delta\left|a_{2} b_{1}\right\rangle\right)_{A B}|\alpha\rangle \\
& \rightarrow\left(\alpha|H H\rangle\left|\alpha \mathrm{e}^{\mathrm{i} \theta}\right\rangle \pm \beta|V V\rangle\left|\alpha \mathrm{e}^{-\mathrm{i} \theta}\right\rangle\right)_{A B} \\
& \quad \otimes\left(\gamma\left|a_{1} b_{2}\right\rangle \pm \delta\left|a_{2} b_{1}\right\rangle\right), \\
& (\alpha|H V\rangle \pm \beta|V H\rangle) \otimes\left(\gamma\left|a_{1} b_{1}\right\rangle \pm \delta\left|a_{2} b_{2}\right\rangle\right)_{A B}|\alpha\rangle \\
& \rightarrow(\alpha|H V\rangle|\alpha\rangle \pm \beta|V H\rangle|\alpha\rangle)_{A B} \\
& \quad \otimes\left(\gamma\left|a_{1} b_{1}\right\rangle \pm \delta\left|a_{2} b_{2}\right\rangle\right), \\
& (\alpha|H V\rangle \pm \beta|V H\rangle) \otimes\left(\gamma\left|a_{1} b_{2}\right\rangle \pm \delta\left|a_{2} b_{1}\right\rangle\right)_{A B}|\alpha\rangle \\
& \rightarrow(\alpha|H V\rangle|\alpha\rangle \pm \beta|V H\rangle|\alpha\rangle)_{A B} \\
& \quad \otimes\left(\gamma\left|a_{1} b_{2}\right\rangle \pm \delta\left|a_{2} b_{1}\right\rangle\right) .
\end{aligned}
$$

After the X-quadrature measurement is performed on the coherent beam, the even-parity polarization Bell states can be distinguished from the odd-parity polarization Bell states. If the coherent state has a phase shift $\theta(-\theta)$, the polarization state of the two-photon system $A B$ is in an even-parity mode. If the coherent state has no phase shift, the polarization state of the two-photon system $A B$ is in the odd-parity mode.

$S-Q N D$ - The setup of the S-QND in hyper-ECP is the same as the one in Fig. 10, That is,

$$
\begin{aligned}
& (\alpha|H H\rangle \pm \beta|V V\rangle) \otimes\left(\gamma\left|a_{1} b_{1}\right\rangle \pm \delta\left|a_{2} b_{2}\right\rangle\right)_{A B}|\alpha\rangle \\
& \rightarrow(\alpha|H H\rangle\rangle \pm \beta|V V\rangle)_{A B} \\
& \quad \otimes\left(\gamma\left|a_{1} b_{1}\right\rangle\left|\alpha \mathrm{e}^{\mathrm{i} \theta}\right\rangle \pm \delta\left|a_{2} b_{2}\right\rangle\left|\alpha \mathrm{e}^{-\mathrm{i} \theta}\right\rangle\right), \\
& (\alpha|H H\rangle \pm \beta|V V\rangle) \otimes\left(\gamma\left|a_{1} b_{2}\right\rangle \pm \delta\left|a_{2} b_{1}\right\rangle\right)_{A B}|\alpha\rangle \\
& \rightarrow(\alpha|H H\rangle \pm \beta|V V\rangle)_{A B} \otimes\left(\gamma\left|a_{1} b_{2}\right\rangle|\alpha\rangle \pm \delta\left|a_{2} b_{1}\right\rangle|\alpha\rangle\right), \\
& (\alpha|H V\rangle \pm \beta|V H\rangle) \otimes\left(\gamma\left|a_{1} b_{1}\right\rangle \pm \delta\left|a_{2} b_{2}\right\rangle\right)_{A B}|\alpha\rangle \\
& \rightarrow(\alpha|H V\rangle \pm \beta|V H\rangle)_{A B} \\
& \quad \otimes\left(\gamma\left|a_{1} b_{1}\right\rangle\left|\alpha \mathrm{e}^{\mathrm{i} \theta}\right\rangle \pm \delta\left|a_{2} b_{2}\right\rangle\left|\alpha \mathrm{e}^{-\mathrm{i} \theta}\right\rangle\right), \\
& (\alpha|H V\rangle \pm \beta|V H\rangle) \otimes\left(\gamma\left|a_{1} b_{2}\right\rangle \pm \delta\left|a_{2} b_{1}\right\rangle\right)_{A B}|\alpha\rangle \\
& \rightarrow(\alpha|H V\rangle \pm \beta|V H\rangle)_{A B} \otimes\left(\gamma\left|a_{1} b_{2}\right\rangle|\alpha\rangle \pm \delta\left|a_{2} b_{1}\right\rangle|\alpha\rangle\right) .
\end{aligned}
$$

After the X-quadrature measurement is performed on the coherent beam, the even-parity spatial-mode Bell states can be distinguished from the odd-parity spatial-mode Bell states. If the coherent state has a phase shift $\theta$ $(-\theta)$, the spatial-mode state of two-photon system $A B$ is in an even-parity mode. If the coherent state has no phase shift, the spatial-mode state of two-photon system $A B$ is in an odd-parity mode.

In long-distance quantum communication, the maximally hyperentangled Bell state $|\phi\rangle_{A B}$ may decay to the partially hyperentangled Bell state $\left|\phi_{0}\right\rangle_{A B}$ by the channel noise. Here

$$
\begin{aligned}
|\phi\rangle_{A B} & =\frac{1}{2}(|H H\rangle+|V V\rangle)_{A B} \otimes\left(\left|a_{1} b_{1}\right\rangle+\left|a_{2} b_{2}\right\rangle\right), \\
\left|\phi_{0}\right\rangle_{A B} & =(\alpha|H H\rangle+\beta|V V\rangle)_{A B} \otimes\left(\gamma\left|a_{1} b_{1}\right\rangle+\delta\left|a_{2} b_{2}\right\rangle\right) .
\end{aligned}
$$


Here, the two photons $A$ and $B$ are obtained by Alice and Bob, respectively. $\alpha, \beta, \gamma$, and $\delta$ are four unknown real parameters and they satisfy the relation $|\alpha|^{2}+|\beta|^{2}=|\gamma|^{2}+|\delta|^{2}=1$. The quantum circuit of the hyper-ECP for the partially hyperentangled Bell state $\left|\phi_{0}\right\rangle_{A B}$ is shown in Fig.16. Two identical two-photon systems $A B$ and $C D$ are required in this hyper-ECP. Here, $\left|\phi_{0}\right\rangle_{C D}=(\alpha|H H\rangle+\beta|V V\rangle)_{C D} \otimes\left(\gamma\left|c_{1} d_{1}\right\rangle+\delta\left|c_{2} d_{2}\right\rangle\right)$, and the two photons $C$ and $D$ are obtained by Alice and Bob, respectively.

The first round of the hyper-ECP - The initial state of the four-photon system $A B C D$ is $\left|\Phi_{0}\right\rangle=\left|\phi_{0}\right\rangle_{A B} \otimes$ $\left|\phi_{0}\right\rangle_{C D}$. Alice and Bob can divide the states of the fourphoton systems into two groups in the polarization DOF with P-QND, and they can also divide the states of the four-photon systems into two groups in the spatial-mode DOF with S-QND 47].

(1) After the X-quadrature measurement is performed on the coherent beam, the results of the P-QND and SQND showed that the polarization DOF of the photon pair $A C$ is in an odd-parity mode and the spatial-mode DOF of the photon pair $B D$ is also in an odd-parity mode. The state of the four-photon system $A B C D$ is projected to $\left|\Phi_{1}\right\rangle$ with the probability of $p(1)=4|\alpha \beta \gamma \delta|^{2}$. Here

$$
\begin{aligned}
\left|\Phi_{1}\right\rangle= & \frac{1}{2}(|H H V V\rangle+|V V H H\rangle)_{A B C D} \\
& \otimes\left(\left|a_{2} b_{2} c_{1} d_{1}\right\rangle+\left|a_{1} b_{1} c_{2} d_{2}\right\rangle\right) .
\end{aligned}
$$

Then, Alice and Bob perform the Hadamard operations on the polarization and spatial-mode DOFs of the two photons $C$ and $D$, respectively. If the detection shows that the polarization and spatial-mode DOFs of the two photons $C$ and $D$ are both in the even-parity mode, the photon pair $A B$ in the maximally hyperentangled Bell state $|\phi\rangle_{A B}=\frac{1}{2}(|H H\rangle+|V V\rangle)_{A B}\left(\left|a_{1} b_{1}\right\rangle+\left|a_{2} b_{2}\right\rangle\right)$ is obtained. If the detection shows that the spatial-mode (polarization) DOF of the two photons $C$ and $D$ is in an odd-parity mode, Bob performs a local phase-flip operation $\sigma_{z}^{S}\left(\sigma_{z}^{P}\right)$ on photon $B$ to obtain the state $|\phi\rangle_{A B}$.

(2) After the X-quadrature measurement is performed on the coherent beam, the results of the P-QND and SQND showed that the polarization DOF of the photon pair $A C$ is in an even-parity mode and the spatial-mode DOF of the photon pair $B D$ is also in an even-parity mode. The state of the four-photon system $A B C D$ is projected to $\left|\Phi_{2}\right\rangle$ with the probability of $p^{\prime}(1)_{1}=\left(|\alpha|^{4}+\right.$ $\left.|\beta|^{4}\right)\left(|\gamma|^{4}+|\delta|^{4}\right)$. Here

$$
\begin{aligned}
\left|\Phi_{2}\right\rangle= & \frac{1}{\sqrt{p^{\prime}(1)_{1}}}\left(\alpha^{2}|H H H H\rangle+\beta^{2}|V V V V\rangle\right)_{A B C D} \\
& \otimes\left(\gamma^{2}\left|a_{1} b_{1} c_{1} d_{1}\right\rangle+\delta^{2}\left|a_{2} b_{2} c_{2} d_{2}\right\rangle\right)
\end{aligned}
$$

After Alice and Bob perform the Hadamard operations and detections on the polarization and spatial-mode DOFs of the two photons $C$ and $D$ and the conditional local phase-flip operations $\sigma_{z}^{S}\left(\sigma_{z}^{P}\right)$ on photon $B$, the state of the two-photon system $A B$ is projected to $\left|\phi_{2}\right\rangle_{A B}$. Here

$$
\begin{aligned}
\left|\phi_{2}\right\rangle_{A B}= & \frac{1}{\sqrt{p^{\prime}(1)_{1}}}\left(\alpha^{2}|H H\rangle+\beta^{2}|V V\rangle\right)_{A B} \\
& \otimes\left(\gamma^{2}\left|a_{1} b_{1}\right\rangle+\delta^{2}\left|a_{2} b_{2}\right\rangle\right) .
\end{aligned}
$$

This is a partially hyperentangled Bell state with less entanglement, and it can be distilled to the maximally hyperentangled Bell state with another round of the hyperECP process.

(3) After the X-quadrature measurement is performed on the coherent beam, the results of the P-QND and SQND showed that the polarization DOF of the photon pair $A C$ is in an odd-parity mode and the spatial-mode DOF of the photon pair $B D$ is in an even-parity mode. The state of the four-photon system $A B C D$ is projected to $\left|\Phi_{3}\right\rangle$ with the probability of $p^{\prime}(1)_{2}=2|\alpha \beta|^{2}\left(|\gamma|^{4}+|\delta|^{4}\right)$. Here

$$
\begin{aligned}
\left|\Phi_{3}\right\rangle= & \frac{1}{\sqrt{2\left(|\gamma|^{4}+|\delta|^{4}\right)}}(|H H V V\rangle+|V V H H\rangle)_{A B C D} \\
& \otimes\left(\gamma^{2}\left|a_{1} b_{1} c_{1} d_{1}\right\rangle+\delta^{2}\left|a_{2} b_{2} c_{2} d_{2}\right\rangle\right),
\end{aligned}
$$

After Alice and Bob perform the Hadamard operations and detections on the polarization and spatial-mode DOFs of the two photons $C$ and $D$ and the conditional local phase-flip operations $\sigma_{z}^{S}\left(\sigma_{z}^{P}\right)$ on photon $B$, the state of the two-photon system $A B$ is projected to $\left|\phi_{3}\right\rangle_{A B}$. Here

$$
\begin{aligned}
\left|\phi_{3}\right\rangle_{A B}= & \frac{1}{\sqrt{2\left(|\gamma|^{4}+|\delta|^{4}\right)}}(|H H\rangle+|V V\rangle)_{A B} \\
& \otimes\left(\gamma^{2}\left|a_{1} b_{1}\right\rangle+\delta^{2}\left|a_{2} b_{2}\right\rangle\right) .
\end{aligned}
$$

This is a partially hyperentangled Bell state with the polarization DOF in a maximally entangled Bell state, and it can be distilled to the maximally hyperentangled Bell state with another round of the hyper-ECP process.

(4) After the X-quadrature measurement is performed on the coherent beam, the results of the P-QND and SQND showed that the polarization DOF of the photon pair $A C$ is in an even-parity mode and the spatial-mode DOF of the photon pair $B D$ is in an odd-parity mode. The state of the four-photon system $A B C D$ is projected to $\left|\Phi_{4}\right\rangle$ with the probability of $p^{\prime}(1)_{3}=2|\gamma \delta|^{2}\left(|\alpha|^{4}+|\beta|^{4}\right)$. Here

$$
\begin{aligned}
\left|\Phi_{4}\right\rangle= & \frac{1}{\sqrt{2\left(|\alpha|^{4}+|\beta|^{4}\right)}}\left(\alpha^{2}|H H H H\rangle+\beta^{2}|V V V V\rangle\right)_{A B C D} \\
& \otimes\left(\left|a_{1} b_{1} c_{2} d_{2}\right\rangle+\left|a_{2} b_{2} c_{1} d_{1}\right\rangle\right) .
\end{aligned}
$$

After Alice and Bob perform the Hadamard operations and detections on the polarization and spatial-mode DOFs of the two photons $C$ and $D$ and the conditional local phase-flip operations $\sigma_{z}^{S}\left(\sigma_{z}^{P}\right)$ on photon $B$, the state of the two-photon system $A B$ is projected to $\left|\phi_{4}\right\rangle_{A B}$. Here

$$
\begin{aligned}
\left|\phi_{4}\right\rangle_{A B}= & \frac{1}{\sqrt{2\left(|\alpha|^{4}+|\beta|^{4}\right)}}\left(\alpha^{2}|H H\rangle+\beta^{2}|V V\rangle\right)_{A B} \\
& \otimes\left(\left|a_{1} b_{1}\right\rangle+\left|a_{2} b_{2}\right\rangle\right) .
\end{aligned}
$$




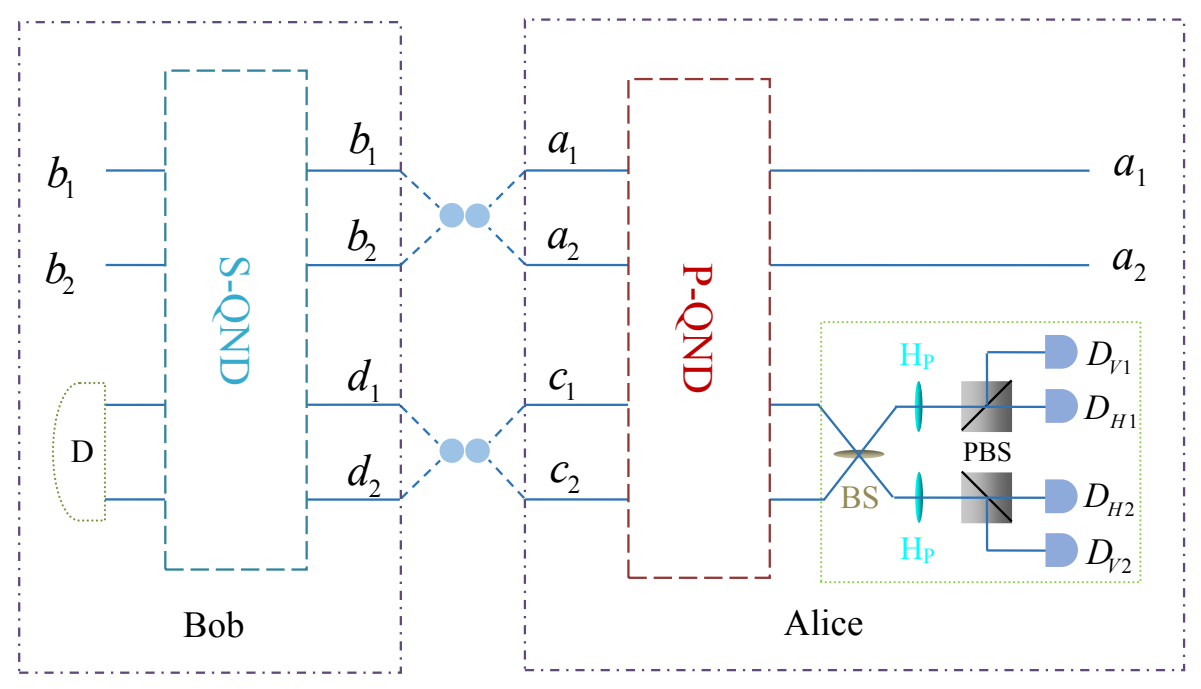

FIG. 16: Schematic diagram of the polarization-spatial hyper-ECP for unknown partially hyperentangled Bell states [48, 51], resorting to the Schmidt projection method. D represents the same operations as the ones performed by Alice in the green dotted box.

This is a partially hyperentangled Bell state with the spatial-mode DOF in a maximally entangled Bell state, and it can be distilled to the maximally hyperentangled Bell state with another round of the hyper-ECP process.

The second round of the hyper-ECP - In the cases (2)-(4) of the first round, the two-photon system $A B$ is projected to a partially hyperentangled Bell state, which requires the second round of the hyper-ECP process.

In the second round of the hyper-ECP process, two identical photon pairs $A B$ and $A^{\prime} B^{\prime}$ are required. Alice and Bob perform the same operations on their photon pairs $A A^{\prime}$ and $B B^{\prime}$ as they did in the first round of the hyper-ECP.

(1') In the case (2) of the first round, the photon pairs $A B$ and $A^{\prime} B^{\prime}$ are in the states $\left|\phi_{2}\right\rangle_{A B}$ and $\left|\phi_{2}\right\rangle_{A^{\prime} B^{\prime}}$. After the P-QND and S-QND are performed on the two-photon systems $A A^{\prime}$ and $B B^{\prime}$, respectively, the four cases in the first round are all obtained. Therefore, after Alice and Bob perform the Hadamard operations and detections on the polarization and spatial-mode DOFs of the two photons $A^{\prime}$ and $B^{\prime}$ and the conditional local phase-flip operations $\sigma_{z}^{S}\left(\sigma_{z}^{P}\right)$ on photon $B$, the maximally hyperentangled Bell state $|\phi\rangle_{A B}$ is obtained with the probability of $p(2)_{1}=4|\alpha \beta \gamma \delta|^{4} /\left[\left(|\alpha|^{4}+|\beta|^{4}\right)\left(|\gamma|^{4}+|\delta|^{4}\right)\right]$. The other three partially hyperentangled Bell states $\left|\phi_{2}\right\rangle_{A B}^{1}$, $\left|\phi_{2}\right\rangle_{A B}^{2}$, and $\left|\phi_{2}\right\rangle_{A B}^{3}$ can be obtained with the probabilities of $p^{\prime}(2)_{1}^{1}=\left(|\alpha|^{8}+|\beta|^{8}\right)\left(|\gamma|^{8}+|\delta|^{8}\right) /\left[\left(|\alpha|^{4}+|\beta|^{4}\right)\left(|\gamma|^{4}+\right.\right.$ $\left.\left.|\delta|^{4}\right)\right], p^{\prime}(2)_{1}^{2}=2|\alpha \beta|^{4}\left(|\gamma|^{8}+|\delta|^{8}\right) /\left[\left(|\alpha|^{4}+|\beta|^{4}\right)\left(|\gamma|^{4}+\right.\right.$ $\left.\left.|\delta|^{4}\right)\right]$, and $p^{\prime}(2)_{1}^{3}=2|\gamma \delta|^{4}\left(|\alpha|^{8}+|\beta|^{8}\right) /\left[\left(|\alpha|^{4}+|\beta|^{4}\right)\left(|\gamma|^{4}+\right.\right.$ $\left.\left.|\delta|^{4}\right)\right]$, respectively. Here

$$
\begin{aligned}
\left|\phi_{2}\right\rangle_{A B}^{1}= & \frac{1}{\sqrt{\left(|\alpha|^{8}+|\beta|^{8}\right)\left(|\gamma|^{8}+|\delta|^{8}\right)}}\left(\alpha^{4}|H H\rangle\right. \\
& \left.+\beta^{4}|V V\rangle\right)_{A B} \otimes\left(\gamma^{4}\left|a_{1} b_{1}\right\rangle+\delta^{4}\left|a_{2} b_{2}\right\rangle\right) \\
\left|\phi_{2}\right\rangle_{A B}^{2}= & \frac{1}{\sqrt{2\left(|\gamma|^{8}+|\delta|^{8}\right)}}(|H H\rangle+|V V\rangle)_{A B} \\
& \otimes\left(\gamma^{4}\left|a_{1} b_{1}\right\rangle+\delta^{4}\left|a_{2} b_{2}\right\rangle\right), \\
\left|\phi_{2}\right\rangle_{A B}^{3}= & \frac{1}{\sqrt{2\left(|\alpha|^{8}+|\beta|^{8}\right)}}\left(\alpha^{4}|H H\rangle+\beta^{4}|V V\rangle\right)_{A B} \\
& \otimes\left(\left|a_{1} b_{1}\right\rangle+\left|a_{2} b_{2}\right\rangle\right)
\end{aligned}
$$

If the photon pair $A B$ is projected to a partially hyperentangled Bell state, another round of the hyper-ECP process is required.

(2') In the case (3) of the first round, the photon pairs $A B$ and $A^{\prime} B^{\prime}$ are in the states $\left|\phi_{3}\right\rangle_{A B}$ and $\left|\phi_{3}\right\rangle_{A^{\prime} B^{\prime}}$. After the P-QND and S-QND are performed on the two-photon systems $A A^{\prime}$ and $B B^{\prime}$, respectively, Alice and Bob pick up the case that the result of S-QND is in an odd-parity mode (the result of P-QND is in either an even-parity mode or an odd-parity mode). After Alice and Bob perform the Hadamard operations and detections on the polarization and spatial-mode DOFs of the two photons $A^{\prime}$ and $B^{\prime}$ and the conditional local phase-flip operations $\sigma_{z}^{S}\left(\sigma_{z}^{P}\right)$ on photon $B$, the maximally hyperentangled Bell state $|\phi\rangle_{A B}$ is obtained with the probability of $p(2)_{2}=4|\gamma \delta|^{4}|\alpha \beta|^{2} /\left(|\gamma|^{4}+|\delta|^{4}\right)$. If the result of S-QND is in an even-parity mode (the result of P-QND is in either an even-parity mode or an odd-parity mode), the partially hyperentangled Bell state $\left|\phi_{3}\right\rangle_{A B}^{1}$ is obtained with the probability of $p^{\prime}(2)_{2}=2\left(|\gamma|^{8}+|\delta|^{8}\right)|\alpha \beta|^{2} /\left(|\gamma|^{4}+|\delta|^{4}\right)$. 
Here

$$
\begin{aligned}
\left|\phi_{3}\right\rangle_{A B}^{1}= & \frac{1}{\sqrt{2\left(|\gamma|^{8}+|\delta|^{8}\right)}}(|H H\rangle+|V V\rangle)_{A B} \\
& \otimes\left(\gamma^{4}\left|a_{1} b_{1}\right\rangle+\delta^{4}\left|a_{2} b_{2}\right\rangle\right) .
\end{aligned}
$$

If the photon pair $A B$ is projected to a partially hyperentangled Bell state, another round of the hyper-ECP process is required.

(3') In the case (4) of the first round, the photon pairs $A B$ and $A^{\prime} B^{\prime}$ are in the states $\left|\phi_{4}\right\rangle_{A B}$ and $\left|\phi_{4}\right\rangle_{A^{\prime} B^{\prime}}$. After the P-QND and S-QND are performed on the two-photon systems $A A^{\prime}$ and $B B^{\prime}$, respectively, Alice and Bob pick up the case that the result of P-QND is in an odd-parity mode (the result of S-QND is in either an even-parity mode or an odd-parity mode). After Alice and Bob perform the Hadamard operations and detections on the polarization and spatial-mode DOFs of the two photons $A^{\prime}$ and $B^{\prime}$ and the conditional local phase-flip operations $\sigma_{z}^{S}\left(\sigma_{z}^{P}\right)$ on photon $B$, the maximally hyperentangled Bell state $|\phi\rangle_{A B}$ is obtained with the probability of $p(2)_{3}=4|\alpha \beta|^{4}|\gamma \delta|^{2} /\left(|\alpha|^{4}+|\beta|^{4}\right)$. If the result of P-QND is in an even-parity mode (the result of S-QND is in either an even-parity mode or an odd-parity mode), the partially hyperentangled Bell state $\left|\phi_{4}\right\rangle_{A B}^{1}$ is obtained with the probability of $p^{\prime}(2)_{3}=2\left(|\alpha|^{8}+|\beta|^{8}\right)|\gamma \delta|^{2} /\left(|\alpha|^{4}+|\beta|^{4}\right)$. Here

$$
\begin{aligned}
\left|\phi_{4}\right\rangle_{A B}^{1}= & \frac{1}{\sqrt{2\left(|\alpha|^{8}+|\beta|^{8}\right)}}\left(\alpha^{4}|H H\rangle+\beta^{4}|V V\rangle\right)_{A B} \\
& \otimes\left(\left|a_{1} b_{1}\right\rangle+\left|a_{2} b_{2}\right\rangle\right) .
\end{aligned}
$$

If the photon pair $A B$ is projected to a partially hyperentangled Bell state, another round of the hyper-ECP process is required.

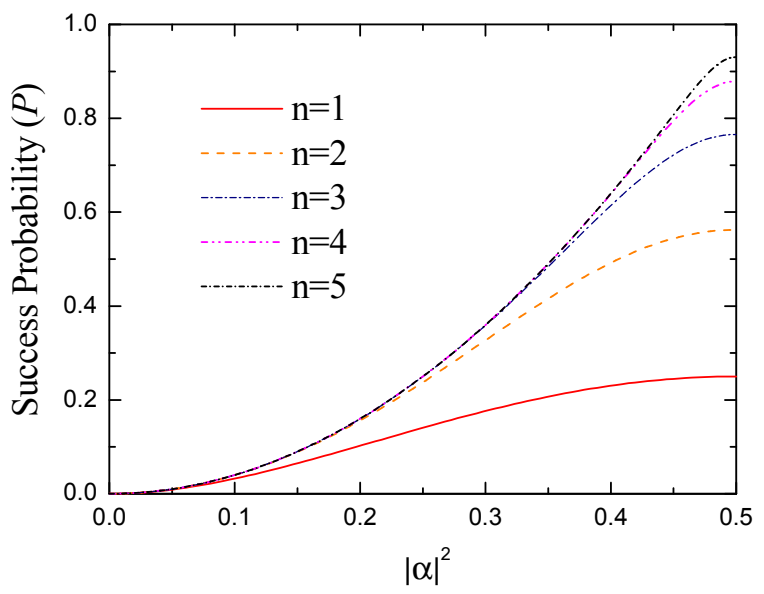

FIG. 17: Success probability of the hyper-ECP for a pair of partially hyperentangled Bell states under the iteration numbers $(n)$ [47, 51]. The parameters of the polarization-spatial partially hyperentangled Bell state are chosen as $|\alpha|=|\gamma|$ and $|\beta|=|\delta|$.
Success probability - The success probabilities of each round of the hyper-ECP process are $p(1), p(2)=p(2)_{1}+$ $p(2)_{2}+p(2)_{3}, \ldots$, respectively. After $n$ rounds of the hyper-ECP process, the total success probability of the hyper-ECP is $P=\sum_{n} p(n)$. The relation between the success probability $P$ and the parameter $|\alpha|^{2}$ under the iteration numbers $n$ is shown in Fig. 17 for the case with $|\alpha|<|\beta|(|\alpha|=|\gamma|$ and $|\beta|=|\delta|)$. It shows that the success probability of the hyper-ECP is improved largely by iterative application of the hyper-ECP process several times.

Now, we can see that the success probability of the hyper-ECP is improved by using the parity-check QNDs, resorting to nonlinear optical elements. The P-QND and S-QND in this protocol can also be constructed with other nonlinear optical elements with similar effect, such as cavity-NV-center system [47], quantum-dot-cavity system [50], and so on. In fact, this hyper-ECP is implemented by concentrating the polarization and spatialmode DOFs independently. If the swap gate is introduced, the success probability of each round of the hyperECP will be greatly improved by transferring the useful information between the nonlocal partially hyperentangled Bell states in cases (3) and (4) in the first round of the hyper-ECP. The detail of the highly efficient twostep hyper-ECP with quantum swap gates is introduced in Ref. [49].

\section{Hyper-ECP for polarization-time-bin hyperentangled Bell state}

The time-bin DOF is a simple and conventional DOF, and it is also very useful in quantum information processing. Here, we introduce a hyper-ECP for unknown polarization-time-bin hyperentangled Bell state with the Schmidt projection method [52].

Two nonlocal photon pairs $A B$ and $C D$ are required in this proposal, and they are in the partially hyperentangled states $|\phi\rangle_{A B}$ and $|\phi\rangle_{C D}$, respectively. Here,

$$
\begin{aligned}
& |\phi\rangle_{A B}=(\alpha|H H\rangle+\beta|V V\rangle) \otimes(\delta|S S\rangle+\eta|L L\rangle)_{A B}, \\
& |\phi\rangle_{C D}=(\alpha|H H\rangle+\beta|V V\rangle) \otimes(\delta|S S\rangle+\eta|L L\rangle)_{C D} .
\end{aligned}
$$

Here $|S\rangle$ and $|L\rangle$ represent two time-bins early and late, respectively, and there is a time interval $\Delta t$ between the two time-bins. The photons $A$ and $C$ belong to Alice, and the photons $B$ and $D$ belong to Bob. $\alpha, \beta, \delta$, and $\eta$ are four unknown parameters, and they satisfy the normalization condition $|\alpha|^{2}+|\beta|^{2}=|\delta|^{2}+|\eta|^{2}=1$.

The initial state of the four-photon system $A B C D$ is $|\Phi\rangle_{0}=|\phi\rangle_{A B} \otimes|\phi\rangle_{C D}$. First, Alice and Bob perform the polarization bit-flip operations $\left(\sigma_{x}^{P}\right)$ with half-wave plates $(\mathrm{X})$ and the time-bin bit-flip operations $\left(\sigma_{x}^{T}=\right.$ 


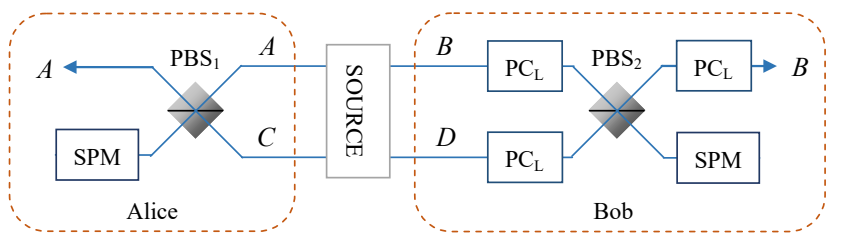

(a)

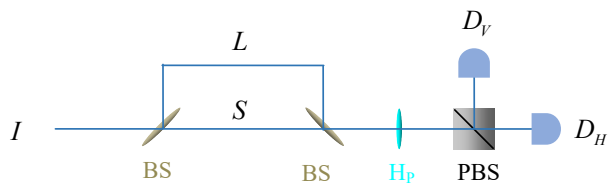

(b)

FIG. 18: (a) Schematic diagram of the polarization-time-bin hyper-ECP for partially hyperentangled Bell states with unknown parameters [52], resorting to the Schmidt projection method. $\mathrm{PC}_{L}\left(\mathrm{PC}_{S}\right)$ represents a Pockels cell, which is used to perform polarization bit-flip operation on the $L(S)$ component. (b) Schematic diagram of a single-photon measurement (SPM).

$|S\rangle\langle L|+| L\rangle\langle S|)$ with the active switches on the two photons $C$ and $D$. The state of the four-photon system $A B C D$ is transformed into $|\Phi\rangle_{1}$. Here

$$
\begin{aligned}
|\Phi\rangle_{1}= & {\left[\left(\alpha^{2}|H H V V\rangle+\beta^{2}|V V H H\rangle\right)\right.} \\
& +\alpha \beta(|H H H H\rangle+|V V V V\rangle)] \\
& \otimes\left[\left(\delta^{2}|S S L L\rangle+\eta^{2}|L L S S\rangle\right)\right. \\
& +\delta \eta(|S S S S\rangle+|L L L L\rangle)]_{A B C D} .
\end{aligned}
$$

Subsequently, Alice and Bob put the photon pairs $A C$ and $B D$ into the quantum circuit shown in Fig. 18 $\mathrm{a}$. That is, Alice put the two photons $A$ and $C$ into the polarization beam splitter $\mathrm{PBS}_{1}$, which can perform a parity-check measurement on the polarization DOF of the photon pair $A C$. Alice picks up the even-parity polarization state with only one photon exiting from each output port of $\mathrm{PBS}_{1}$, and the state of the four-photon system will be transformed from $|\Phi\rangle_{1}$ to $|\Phi\rangle_{2}$ with the probability of $2|\alpha \beta|^{2}$. Here

$$
\begin{aligned}
|\Phi\rangle_{2}= & \frac{1}{\sqrt{2}}(|H H H H\rangle+|V V V V\rangle) \\
& \otimes\left[\left(\delta^{2}|S S L L\rangle+\eta^{2}|L L S S\rangle\right)\right. \\
& +\delta \eta(|S S S S\rangle+|L L L L\rangle)]_{A B C D}
\end{aligned}
$$

At the same time, Bob puts the two photons $B$ and $D$ into two Pockels cells (PCs), which can perform the polarization bit-flip operations on the photon system at a specific time. That is, $\mathrm{PC}_{L}\left(\mathrm{PC}_{S}\right)$ only performs the polarization bit-flip operation on the $L(S)$ component. Then the state of the four-photon system is changed from
$|\Phi\rangle_{2}$ to $|\Phi\rangle_{3}$. Here

$$
\begin{aligned}
|\Phi\rangle_{3}= & \frac{1}{\sqrt{2}}\left[\delta^{2}\left(\left|H^{S} H^{S} H^{L} V^{L}\right\rangle+\left|V^{S} V^{S} V^{L} H^{L}\right\rangle\right)\right. \\
& +\eta^{2}\left(\left|H^{L} V^{L} H^{S} H^{S}\right\rangle+\left|V^{L} H^{L} V^{S} V^{S}\right\rangle\right) \\
& +\delta \eta\left(\left|H^{S} H^{S} H^{S} H^{S}\right\rangle+\left|V^{S} V^{S} V^{S} V^{S}\right\rangle\right. \\
& \left.\left.+\left|H^{L} V^{L} H^{L} V^{L}\right\rangle+\left|V^{L} H^{L} V^{L} H^{L}\right\rangle\right)\right]_{A B C D} .
\end{aligned}
$$

The superscript $L(S)$ represents the time-bin late (early). Then Bob can perform the time-bin parity-check measurement on the two photons $B$ and $D$ with $\mathrm{PBS}_{2}$, and he also picks up the even-parity polarization state with only one photon exiting from each output port of $\mathrm{PBS}_{2}$. After another $\mathrm{PC}_{L}$ is performed on photon $B$, the four-photon system will be projected into the state $|\Phi\rangle_{4}$ with the probability of $4|\alpha \beta \delta \eta|^{2}$. Here

$$
\begin{aligned}
|\Phi\rangle_{4}= & \frac{1}{2}\left(\left|H^{S} H^{S} H^{S} H^{S}\right\rangle+\left|V^{S} V^{S} V^{S} V^{S}\right\rangle\right. \\
& \left.+\left|H^{L} H^{L} H^{L} V^{L}\right\rangle+\left|V^{L} V^{L} V^{L} H^{L}\right\rangle\right)_{A B C D} .
\end{aligned}
$$

The last step of this hyper-ECP is to detect the two photons $C$ and $D$ with the single-photon measurement (SPM) shown in Fig. 18b, which can transform the state $|\Phi\rangle_{4}$ to one of the maximally hyperentangled Bell states $\left|\psi^{ \pm \pm}\right\rangle_{A B}$. Here

$$
\left|\psi^{ \pm \pm}\right\rangle_{A B}=\frac{1}{2}(|H H\rangle \pm|V V\rangle)_{A B} \otimes(|S S\rangle \pm|L L\rangle)_{A B} .
$$

In the quantum circuit of SPM shown in Fig. $18 \mathrm{~b}$, the length difference between the two arms of beam splitters $\left(\mathrm{BS}_{1}\right.$ and $\left.\mathrm{BS}_{2}\right)$ is $c \Delta t$, which is used as an unbalanced interferometer (UI). Here $c$ is the speed of the photons. The effect of this UI is

$$
\begin{aligned}
& \left|X^{L}\right\rangle \rightarrow \frac{1}{\sqrt{2}}\left(\left|X^{L S}\right\rangle+\left|X^{L L}\right\rangle\right), \\
& \left|X^{S}\right\rangle \rightarrow \frac{1}{\sqrt{2}}\left(\left|X^{S S}\right\rangle+\left|X^{S L}\right\rangle\right) .
\end{aligned}
$$

Here, $X$ represents $H$ or $V$, and $X^{i j}(i, j=L, S)$ represents the time-bin state $X^{i}$ passing through the arm $j$ of the UI. After the two photons $C$ and $D$ pass through UI, the state of the four-photon system is changed to

$$
\begin{aligned}
& \left|H^{S} H^{S}\right\rangle_{A B} \otimes\left(\left|H^{S S}\right\rangle+\left|H^{S L}\right\rangle\right)_{C} \otimes\left(\left|H^{S S}\right\rangle+\left|H^{S L}\right\rangle\right)_{D} \\
& +\left|V^{S} V^{S}\right\rangle_{A B} \otimes\left(\left|V^{S S}\right\rangle+\left|V^{S L}\right\rangle\right)_{C} \otimes\left(\left|V^{S S}\right\rangle+\left|V^{S L}\right\rangle\right)_{D} \\
& +\left|H^{L} H^{L}\right\rangle_{A B} \otimes\left(\left|H^{L S}\right\rangle+\left|H^{L L}\right\rangle\right)_{C} \otimes\left(\left|H^{L S}\right\rangle+\left|H^{L L}\right\rangle\right)_{D} \\
& +\left|V^{L} V^{L}\right\rangle_{A B} \otimes\left(\left|V^{L S}\right\rangle+\left|V^{L L}\right\rangle\right)_{C} \otimes\left(\left|V^{L S}\right\rangle+\left|V^{L L}\right\rangle\right)_{D} .
\end{aligned}
$$

The time-bin components $L S$ and $S L$ will arrive at the same time, and the time-bin components $L L$ and $S S$ will 
arrive at a later time and an earlier time, respectively. In order to obtain the maximally hyperentangled Bell state, Alice and Bob only pick up the states arriving at the middle time $L S$ and $S L$. After the two photons $C$ and $D$ are detected, the local polarization operation and time-bin operation have to be performed on the photon $B$ to obtain the maximally hyperentangled Bell state $\left|\psi^{++}\right\rangle_{A B}$, which is shown in Table 1 .

TABLE I: The relation between measurement results of $C D$ in the middle time slot, the final states of $A B$, and the local operations to obtain the maximally hyperentangled Bell state $\left|\psi^{++}\right\rangle_{A B}$

\begin{tabular}{ccc}
\hline Detection $(C D)$ & State of $A B$ & Local operation \\
\hline$H H$ & $\left|\psi^{++}\right\rangle^{A B}$ & $I$ \\
$H V$ & $\left|\psi^{--}\right\rangle^{A B}$ & $\sigma_{z}^{T}, \sigma_{z}^{P}$ \\
$V H$ & $\left|\psi^{-+}\right\rangle^{A B}$ & $\sigma_{z}^{P}$ \\
$V V$ & $\left|\psi^{+-}\right\rangle^{A B}$ & $\sigma_{z}^{T}$ \\
\hline
\end{tabular}

The success probability of this hyper-ECP is $P=$ $|\alpha \beta \gamma \delta|^{2}$, which is a quarter of the one for the polarizationspatial hyperentangled Bell sate. An improved SPM is also introduced by $\mathrm{Li}$ and Ghose 52 . With the improved SPM, the success probability of this hyper-ECP can be enhanced to $P=4|\alpha \beta \gamma \delta|^{2}$.

\section{HYPERENTANGLEMENT PURIFICATION}

\section{A. History of entanglement purification}

Entanglement purification is an important passive way for depressing the influence of noise on nonlocal quantum systems and it is an indispensable technique in quantum repeaters. Generally speaking, it is used to distill some nonlocal entangled systems in a high-fidelity entangled state from a set of nonlocal entangled systems in a mixed state with less entanglement 87]. In 1996, Bennett et al. [87] presented the first entanglement purification protocol (EPP) which is used to purify a Werner state based on quantum controlled-not (CNOT) gates. Subsequently, Deutsch et al. 88] improved this EPP by adding two special unitary operations for each particle. In 2001, Pan, Simon, and Zellinger 89 proposed an EPP with linearoptics elements for ideal entangled photon sources. In 2002, Simon and Pan 90 proposed an EPP for two entangled photons from a SPDC source with two PBSs, and Pan et al. 91 demonstrated this EPP in experiment in 2003. Based on the cross-Kerr nonlinearities, an efficient polarization EPP was proposed by Sheng et al. [92] in 2008. In this scheme, the parties in quantum communication can increase the entanglement and improve the fidelity of quantum states by repeatedly performing the purification protocols. In 2011, Wang et al. 93] proposed an interesting EPP using cross-Kerr nonlinearity by identifying the intensity of probe coherent beams. Also, Wang et al. 94 presented an EPP for electron-spin entangled states using quantum-dot spin and microcavity coupled systems. In 2013, Sheng et al. 95] presented an hybrid EPP for quantum repeaters. In 2016, Zhou and Sheng [96] proposed an EPP for logic-qubit entanglement.

Entanglement purification makes great progress since the concept of deterministic entanglement purification was introduced originally by Sheng and Deng [31] in 2010. In this year, they [31] presented a two-step deterministic EPP for polarization entanglement with the hyperentanglement in both the spatial-mode and the frequency DOFs of photon pairs. Subsequently, Sheng and Deng 32] and $\mathrm{Li} 33$ independently proposed the one-step deterministic EPP for polarization entanglement with only the spatial entanglement of photon pairs, resorting to linear-optical elements only. In 2011, Deng [34] extended the deterministic entanglement purification to multipartite entanglement with the spatial entanglement or the frequency entanglement of photon systems. Moreover, he 34] showed that a deterministic EPP does not require the photon systems entangled in the polarization DOF, but one error-free DOF. These one-step deterministic EPPs 32 34 can purify the polarization entanglement with one step, resorting to linear-optical elements, and the polarization errors are totally converted into the ambiguity of spatial modes when the two photons in each pair are originally entangled in spatial DOF which has been exploited to produce a $(100 \times 100)$-dimensional entanglement [97]. In 2014, Sheng and Zhou [98] also described another good deterministic EPP for polarization entanglement with time-bin entanglement. In 2015, Sheng and Zhou [99] proposed the deterministic entanglement distillation for secure double-server blind quantum computation. The deterministic EPPs [31 34] are far different from the conventional EPPs [87 95] as they work in a completely deterministic way, not in a probabilistic way, and they can reduce the quantum resource sacrificed largely. They are very useful in quantum repeaters.

The purification of nonlocal hyperentangled quantum systems is more complex than that of entangled systems in one DOF. In 2013, Ren and Deng [47] presented the first hyperentanglement purification protocol (hyper-EPP) for two-photon systems in polarizationspatial hyperentangled states, and it is very useful in the high-capacity quantum repeaters with hyperentanglement. In 2014, Ren et al. [54] gave the two-step hyper-EPP for polarization-spatial hyperentangled states with the quantum-state-joining method [100], and it has a far higher efficiency. In 2016, Wang et al. [55] presented the first hyper-EPP for two-photon six-qubit hyperentangled systems in three DOFs, and they showed that using SWAP gates is a universal method for hyper-EPP for nonlocal hyperentangled quantum states in both the polarization DOF and multiple-longitudinal-momentum DOFs to obtain a high yield (efficiency), as well as other hyperentanglement with more than two DOFs. In 2015, Wang et al. 56, 57 proposed two novel hyper-EPPs by combining both the conventional EPP and photon-loss 
amplification on hyperentangled photon systems assisted by local entanglement resource.

\section{B. Two-step hyper-EPP}

Here, we introduce the principle of the two-step hyperEPP [54 for polarization-spatial hyperentangled Bell state with the quantum-state-joining method (QSJM).

For simplification, we only discuss the principle of the two-step hyper-EPP for mixed hyperentangled Bell states with polarization bit-flip errors and spatial-mode phase-flip errors [54], resorting to the polarization-spatial phase-check QND (P-S-QND) and QSJM. This hyperEPP can be used for nonlocally mixed hyperentangled Bell states with arbitrary errors in the two DOFs. In this hyper-EPP, the P-S-QND is used to distinguish the Bell state with a relative phase 0 from the one with a relative phase $\pi$ in both the polarization and the spatial-mode DOFs. The QSJM is used to combine the polarization state of photon $A$ and the spatial-mode state of photon $B$ into an output single-photon state. Both these two basic gate elements are constructed with the nonlinearity of a quantum dot (QD) embedded in a double-sided optical microcavity (double-sided QD-cavity system, shown in Fig. [19a).

The input-output optical property of the double-sided $Q D$-cavity system - The two distributed Bragg reflectors of the double-sided optical microcavity are partially reflective, and they are low loss for on-resonance transmission in both the two polarization modes. When an excess electron is injected into a QD [101], the negatively charged exciton $X^{-}$, which consists of two antiparallel electrons bound to one hole, will be created by resonantly absorbing a circularly polarized light, according to the spin-dependent transition rule with Pauli's exclusion principle 102, 103] (shown in Fig. 19b). That is, a circularly polarized photon with the spin $S_{z}=+1$ is absorbed to create the negatively charged exciton $X^{-}$in the state $|\uparrow \downarrow \uparrow\rangle$ for the excess electron spin $|\uparrow\rangle$, and a circularly polarized photon with the $\operatorname{spin} S_{z}=-1$ is absorbed to create the negatively charged exciton $X^{-}$in the state $|\downarrow \uparrow \downarrow\rangle$ for the excess electron spin $|\downarrow\rangle$. The state $|\Uparrow\rangle(|\Downarrow\rangle)$ represents the heavy-hole spin $\left|+\frac{3}{2}\right\rangle$ $\left(\left|-\frac{3}{2}\right\rangle\right)$, and the state $|\uparrow\rangle(|\downarrow\rangle)$ represents the electron $\operatorname{spin}\left|+\frac{1}{2}\right\rangle\left(\left|-\frac{1}{2}\right\rangle\right)$.

The input-output optical property of the double-sided QD-cavity system can be described by the Heisenberg equations of motion for the cavity field operator $\hat{a}$ and $X^{-}$dipole operator $\hat{\sigma}_{-}$in the interaction picture 104,

$$
\begin{aligned}
\frac{\mathrm{d} \hat{a}}{\mathrm{~d} t} & =-\left[\mathrm{i}\left(\omega_{\mathrm{c}}-\omega\right)+\kappa+\frac{\kappa_{\mathrm{s}}}{2}\right] \hat{a}-g \hat{\sigma}_{-}-\sqrt{\kappa} \hat{a}_{\mathrm{in}}-\sqrt{\kappa} \hat{a}_{\mathrm{in}}^{\prime} \\
\frac{\mathrm{d} \hat{\sigma}_{-}}{\mathrm{d} t} & =-\left[\mathrm{i}\left(\omega_{X^{-}}-\omega\right)+\frac{\gamma}{2}\right] \hat{\sigma}_{-}-g \hat{\sigma}_{z} \hat{a}
\end{aligned}
$$

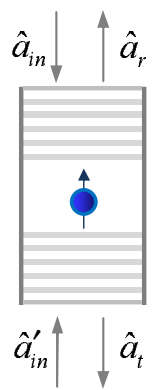

(a)

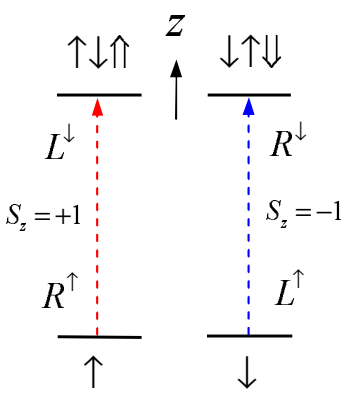

(b)
FIG. 19: (a) A double-sided QD-cavity system. (b) The spindependent optical transitions of a negatively charged exciton $X^{-}$with circularly polarized photons 54]. $L^{\uparrow}\left(L^{\downarrow}\right)$ and $R^{\uparrow}$ $\left(R^{\downarrow}\right)$ represent the left and the right circularly polarized lights with their input directions parallel (antiparallel) with z direction, respectively.

where $\omega, \omega_{\mathrm{c}}$, and $\omega_{X^{-}}$are the frequencies of the input photon, the cavity field mode, and the $X^{-}$transition, respectively. $\kappa$ and $\kappa_{\mathrm{S}} / 2$ are the decay rates of the cavity field mode to the output photon and the side leakage mode, respectively. $g$ is the coupling strength of the negatively charged exciton $X^{-}$and the cavity field mode. $\gamma / 2$ is the decay rate of negatively charged exciton $X^{-} . \quad \hat{a}_{\text {in }}$ and $\hat{a}_{\text {in }}^{\prime}$ are the input field operators of the double-sided QD-cavity system. $\hat{a}_{r}$ and $\hat{a}_{t}$ are the output field operators of the double-sided QD-cavity system. These field operators satisfy the boundary condition $\hat{a}_{r}=\hat{a}_{\text {in }}+\sqrt{\kappa} \hat{a}$ and $\hat{a}_{t}=\hat{a}_{\text {in }}^{\prime}+\sqrt{\kappa} \hat{a}$. In the weak excitation limit $\left(\left\langle\hat{\sigma}_{z}\right\rangle=-1\right)$, the reflection coefficient $(r(\omega))$ and transmission coefficient $(t(\omega))$ of the double-sided QD-cavity system can be expressed as [105, 106]

$$
\begin{aligned}
r(\omega) & =1+t(\omega), \\
t(\omega) & =\frac{-\kappa\left[\mathrm{i}\left(\omega_{X^{-}}-\omega\right)+\frac{\gamma}{2}\right]}{\left[\mathrm{i}\left(\omega_{X^{-}}-\omega\right)+\frac{\gamma}{2}\right]\left[\mathrm{i}\left(\omega_{\mathrm{c}}-\omega\right)+\kappa+\frac{\kappa_{\mathrm{s}}}{2}\right]+g^{2}} .
\end{aligned}
$$

In the resonant condition $\left(\omega_{\mathrm{c}}=\omega_{X^{-}}=\omega\right)$ with $\kappa_{\mathrm{S}} \rightarrow 0$, the reflection and transmission coefficients are $r_{0} \rightarrow 0$ and $t_{0} \rightarrow-1$ for $g=0$, and they are $r \rightarrow 1$ and $t \rightarrow 0$ for the strong coupling regime $g>(\kappa, \gamma)$. As the photonic circular polarization is dependent on the direction of propagation, the photon with spin $S_{z}=+1$ corresponds to the state $\left|R^{\uparrow}\right\rangle$ or $\left|L^{\downarrow}\right\rangle$, and the photon with spin $S_{z}=-1$ corresponds to the state $\left|R^{\downarrow}\right\rangle$ or $\left|L^{\uparrow}\right\rangle$. Here $L^{\uparrow}\left(R^{\uparrow}\right)$ or $L^{\downarrow}\left(R^{\downarrow}\right)$ represents the input direction of the left (right) circularly polarized light which is parallel or antiparallel to the $\mathrm{z}$ direction, respectively, as shown in Fig. 19b. In this condition, the reflection and transmission rules of the photonic polarization states are described as follows,

$$
\begin{array}{ll}
\left|R^{\uparrow}, i_{2}, \uparrow\right\rangle \rightarrow\left|L^{\downarrow}, i_{2}, \uparrow\right\rangle, & \left|L^{\downarrow}, i_{1}, \uparrow\right\rangle \rightarrow\left|R^{\uparrow}, i_{1}, \uparrow\right\rangle, \\
\left|R^{\uparrow}, i_{2}, \downarrow\right\rangle \rightarrow-\left|R^{\uparrow}, i_{1} \downarrow\right\rangle, & \left|L^{\downarrow}, i_{1}, \downarrow\right\rangle \rightarrow-\left|L^{\downarrow}, i_{2}, \downarrow\right\rangle, \\
\left|R^{\downarrow}, i_{1}, \uparrow\right\rangle \rightarrow-\left|R^{\downarrow}, i_{2}, \uparrow\right\rangle, & \left|L^{\uparrow}, i_{2}, \uparrow\right\rangle \rightarrow-\left|L^{\uparrow}, i_{1}, \uparrow\right\rangle, \\
\left|R^{\downarrow}, i_{1}, \downarrow\right\rangle \rightarrow\left|L^{\uparrow}, i_{1}, \downarrow\right\rangle, & \left|L^{\uparrow}, i_{2}, \downarrow\right\rangle \rightarrow\left|R^{\downarrow}, i_{2}, \downarrow\right\rangle .
\end{array}
$$




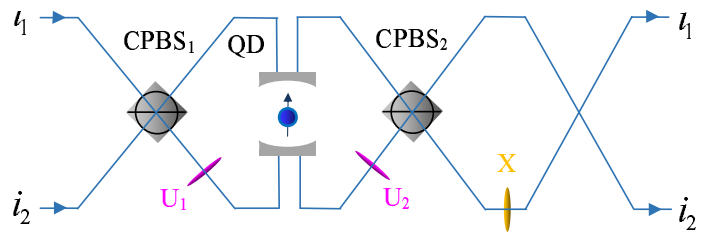

(a)

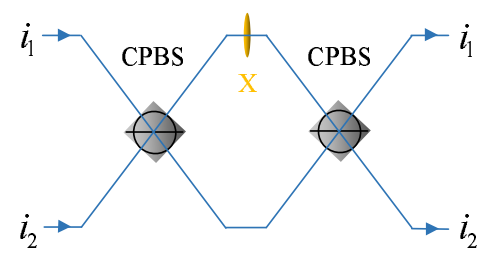

(b)

FIG. 20: (a) Schematic diagram of the quantum-state-joining method (QSJM) 54]. CPBS represents a polarizing beam splitter in the circular basis, which transmits the photon in the right-circular polarization $|R\rangle$ and reflects the photon in the left-circular polarization $|L\rangle$, respectively. $\mathrm{U}_{i}(i=1,2)$ represents a wave plate which is used to perform a polarization phase-flip operation $U=-|R\rangle\langle R|-| L\rangle\langle L|$ on a photon. $\mathrm{X}$ represents a half-wave plate which is used to perform a polarization bit-flip operation $\sigma_{x}^{P}=|R\rangle\langle L|+| L\rangle\langle R|$. (b) Schematic diagram of the swap gate between the spatial-mode state and the polarization state of a photon.

Quantum-state-joining method - The QSJM is used to combine the polarization state of photon $A$ and the spatial-mode state of photon $B$ into an output single photon state. That is, the polarization state of photon $A$ is transferred to the polarization DOF of photon $B$. This QSJM is constructed with the nonlinearity of a double-sided QD-cavity system [54], as shown in Fig. 20k. The initial state of the excess electron spin in QD is $\frac{1}{\sqrt{2}}(|\uparrow\rangle+|\downarrow\rangle)_{e}$. The initial states of two photons $A$ and $B$ are $\left|\varphi_{A}\right\rangle=\left(\alpha_{1}|R\rangle+\alpha_{2}|L\rangle\right)_{A}\left(\gamma_{1}\left|a_{1}\right\rangle+\gamma_{2}\left|a_{2}\right\rangle\right)$ and $\left|\varphi_{B}\right\rangle=\left(\beta_{1}|R\rangle+\beta_{2}|L\rangle\right)_{B}\left(\delta_{1}\left|b_{1}\right\rangle+\delta_{2}\left|b_{2}\right\rangle\right)$, respectively.

First, the two spatial modes of photon $A$ are put into $\mathrm{CPBS}_{1}, \mathrm{U}_{1}, \mathrm{QD}, \mathrm{U}_{2}, \mathrm{CPBS}_{2}$, and $\mathrm{X}$ in sequence, as shown in Fig. 20a. The state of the system $A e$ is transformed from $\left|\varphi_{A e}\right\rangle_{0}$ to $\left|\varphi_{A e}\right\rangle_{1}$. Here

$$
\begin{aligned}
\left|\varphi_{A e}\right\rangle_{0}= & |+\rangle_{e} \otimes\left|\varphi_{A}\right\rangle, \\
\left|\varphi_{A e}\right\rangle_{1}= & \frac{1}{\sqrt{2}}\left[|R\rangle_{A}\left(\alpha_{1}|\uparrow\rangle+\alpha_{2}|\downarrow\rangle\right)_{e}+|L\rangle_{A}\left(\alpha_{2}|\uparrow\rangle\right.\right. \\
& \left.\left.+\alpha_{1}|\downarrow\rangle\right)_{e}\right]\left(\gamma_{1}\left|a_{1}\right\rangle+\gamma_{2}\left|a_{2}\right\rangle\right) .
\end{aligned}
$$

Then the polarization state of photon $A$ is measured in the orthogonal basis $\{|R\rangle,|L\rangle\}$. If the polarization state of photon $A$ is projected to $|R\rangle$, the excess electron spin state in $\mathrm{QD}$ is $|\phi\rangle_{e}=\left(\alpha_{1}|\uparrow\rangle+\alpha_{2}|\downarrow\rangle\right)_{e}$. Otherwise, the excess electron spin state in QD is $\left|\phi^{\prime}\right\rangle_{e}=\left(\alpha_{2}|\uparrow\rangle+\alpha_{1} \mid \downarrow\right.$ \rangle$)_{e}$.

Subsequently, after a Hadamard operation is performed on the excess electron spin $e$ in $\mathrm{QD}$, the two spa- tial modes of photon $B$ are put into the quantum circuit shown in Fig. 201. Here the Hadamard operation on the excess electron spin is $|\uparrow\rangle_{e} \rightarrow \frac{1}{\sqrt{2}}(|\uparrow\rangle+|\downarrow\rangle)_{e},|\downarrow\rangle_{e} \rightarrow$ $\frac{1}{\sqrt{2}}(|\uparrow\rangle-|\downarrow\rangle)_{e}$. Then the state of the system $B e$ is transformed from $\left|\varphi_{B e}\right\rangle_{1}$ to $\left|\varphi_{B e}\right\rangle_{2}$. Here

$$
\begin{aligned}
\left|\varphi_{B e}\right\rangle_{1}= & \left|\varphi_{B}\right\rangle \otimes\left|\phi_{e}\right\rangle \\
\left|\varphi_{B e}\right\rangle_{2}= & {\left[\alpha_{1}^{\prime}|\uparrow\rangle_{e}\left(\beta_{1}|R\rangle+\beta_{2}|L\rangle\right)_{B}+\alpha_{2}^{\prime}|\downarrow\rangle_{e}\left(\beta_{2}|R\rangle\right.\right.} \\
& \left.\left.+\beta_{1}|L\rangle\right)_{B}\right]\left(\delta_{1}\left|b_{1}\right\rangle+\delta_{2}\left|b_{2}\right\rangle\right),
\end{aligned}
$$

where $\alpha_{1}^{\prime}=\frac{1}{\sqrt{2}}\left(\alpha_{1}+\alpha_{2}\right)$ and $\alpha_{2}^{\prime}=\frac{1}{\sqrt{2}}\left(\alpha_{1}-\alpha_{2}\right)$.

Next, the Hadamard operations are performed on the polarization DOF of photon $B$ and the excess electron spin $e$, respectively. Then the two spatial modes of photon $B$ are put into the quantum circuit shown in Fig. 20a again, and the state of the system $B e$ is changed to $\left|\varphi_{B e}\right\rangle_{3}$. Here

$$
\begin{aligned}
\left|\varphi_{B e}\right\rangle_{3}= & {\left[\alpha_{1}|R\rangle_{B}\left(\beta_{1}^{\prime}|\uparrow\rangle+\beta_{2}^{\prime}|\downarrow\rangle\right)_{e}+\alpha_{2}|L\rangle_{B}\left(\beta_{2}^{\prime}|\uparrow\rangle\right.\right.} \\
& \left.\left.+\beta_{1}^{\prime}|\downarrow\rangle\right)_{e}\right] \otimes\left(\delta_{1}\left|b_{1}\right\rangle+\delta_{2}\left|b_{2}\right\rangle\right),
\end{aligned}
$$

where $\beta_{1}^{\prime}=\frac{1}{\sqrt{2}}\left(\beta_{1}+\beta_{2}\right)$ and $\beta_{2}^{\prime}=\frac{1}{\sqrt{2}}\left(\beta_{1}-\beta_{2}\right)$.

Finally, a Hadamard operation is performed on the excess electron spin $e$ again. The state of the system $B e$ is transferred from $\left|\varphi_{B e}\right\rangle_{3}$ to $\left|\varphi_{B e}\right\rangle_{4}$. Here

$$
\begin{aligned}
\left|\varphi_{B e}\right\rangle_{4}= & {\left[\beta_{1}|\uparrow\rangle_{e}\left(\alpha_{1}|R\rangle+\alpha_{2}|L\rangle\right)_{B}+\beta_{2}|\downarrow\rangle_{e}\left(\alpha_{1}|R\rangle\right.\right.} \\
& \left.\left.-\alpha_{2}|L\rangle\right)_{B}\right]\left(\delta_{1}\left|b_{1}\right\rangle+\delta_{2}\left|b_{2}\right\rangle\right) .
\end{aligned}
$$

The excess electron spin $e$ is measured in the orthogonal basis $\{|\uparrow\rangle,|\downarrow\rangle\}$. If the detection shows that the polarization state of photon $A$ is $|L\rangle$, a polarization bitflip operation $\left(\sigma_{x}^{P}=|R\rangle\langle L|+| L\rangle\langle R|\right)$ on photon $B$ is required. If the detection shows that the excess electron spin state is $|\downarrow\rangle_{e}$, a polarization phase-flip operation $\left(\sigma_{z}^{P}=|R\rangle\langle R|-| L\rangle\langle L|\right)$ on photon $B$ is required. With these conditional operations, the final state of photon $B$ is obtained as $\left|\varphi_{B}\right\rangle_{f}=\left(\alpha_{1}|R\rangle+\alpha_{2}|L\rangle\right)_{B}\left(\delta_{1}\left|b_{1}\right\rangle+\delta_{2}\left|b_{2}\right\rangle\right)$. This is just the result of the QSJM. The QSJM for other conditions can be implemented in the same way assisted by the quantum circuit shown in Fig. 20p , such as combining the spatial-mode state of photon $A$ and the spatial-mode state of photon $B$ into an output single photon state.

Polarization-spatial phase-check QND - The P-SQND is used to distinguish the Bell state with a relative phase 0 from the one with a relative phase $\pi$ in both the polarization and spatial-mode DOFs. It is constructed with the hybrid CNOT gate (introduced in Sec. VI) based on the nonlinearity of double-sided QD-cavity systems [54], as shown in Fig. 21]. The states of the excess electron spins $e_{1}$ in $\mathrm{QD}_{1}$ and $e_{2}$ in $\mathrm{QD}_{2}$ are prepared in $|+\rangle_{e_{1}}$ and $|+\rangle_{e_{2}}$, respectively. Here $| \pm\rangle_{e}=\frac{1}{\sqrt{2}}(|\uparrow\rangle \pm|\downarrow\rangle)_{e}$.

After the two photons $A$ and $B$ in the hyperentangled Bell state pass through the quantum circuit shown in 


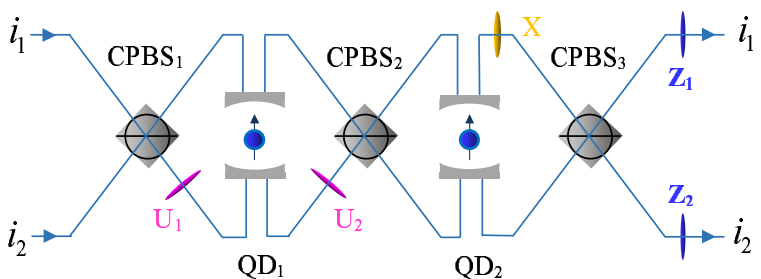

(a)

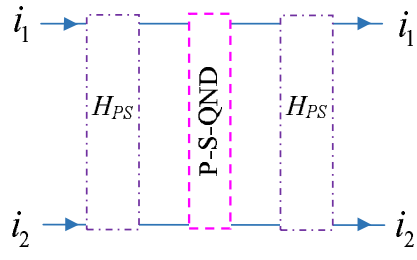

(b)

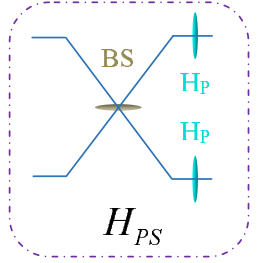

(c)
FIG. 21: (a) Schematic diagram of the polarization-spatial phase-check QND (P-S-QND) 54. $\mathrm{Z}_{i}(i=1,2)$ represents a wave plate which is used to perform a polarization phaseflip operation $\sigma_{z}^{P}=-|R\rangle\langle R|+| L\rangle\langle L|$ on a photon. (b) Schematic diagram of the polarization-spatial parity-check QND. (c) Schematic diagram of $H_{P S}$ which is used to perform the Hadamard operations on both the polarization and spatial-mode DOFs of a photon.

Fig. 213 in sequence, the state of the system $A B e_{1} e_{2}$ evolves to

$$
\begin{aligned}
\left|\phi^{ \pm}\right\rangle_{P}\left|\phi^{ \pm}\right\rangle_{S}|+\rangle_{e_{1}}|+\rangle_{e_{2}} & \rightarrow\left|\phi^{ \pm}\right\rangle_{P}\left|\phi^{ \pm}\right\rangle_{S}| \pm\rangle_{e_{1}}|\mp\rangle_{e_{2}}, \\
\left|\phi^{ \pm}\right\rangle_{P}\left|\psi^{ \pm}\right\rangle_{S}|+\rangle_{e_{1}}|+\rangle_{e_{2}} & \rightarrow\left|\phi^{ \pm}\right\rangle_{P}\left|\psi^{ \pm}\right\rangle_{S}| \pm\rangle_{e_{1}}|\mp\rangle_{e_{2}}, \\
\left|\psi^{ \pm}\right\rangle_{P}\left|\phi^{ \pm}\right\rangle_{S}|+\rangle_{e_{1}}|+\rangle_{e_{2}} & \rightarrow\left|\psi^{ \pm}\right\rangle_{P}\left|\phi^{ \pm}\right\rangle_{S}| \pm\rangle_{e_{1}}|\mp\rangle_{e_{2}}, \\
\left|\psi^{ \pm}\right\rangle_{P}\left|\psi^{ \pm}\right\rangle_{S}|+\rangle_{e_{1}}|+\rangle_{e_{2}} & \rightarrow\left|\psi^{ \pm}\right\rangle_{P}\left|\psi^{ \pm}\right\rangle_{S}| \pm\rangle_{e_{1}}|\mp\rangle_{e_{2}} .
\end{aligned}
$$

Then the excess electron spins $e_{1}$ and $e_{2}$ are measured in the orthogonal basis $\left\{|+\rangle_{e},|-\rangle_{e}\right\}$. If the state of the excess electron spin $e_{1}$ is $|+\rangle_{e_{1}}$, the relative phase of the polarization state is 0 . If the state of the excess electron spin $e_{1}$ is $|-\rangle_{e_{1}}$, the relative phase of the polarization state is $\pi$. If the state of the excess electron spin $e_{2}$ is $|-\rangle_{e_{2}}$, the relative phase of the spatial-mode state is 0 . If the state of the excess electron spin $e_{2}$ is $|+\rangle_{e_{2}}$, the relative phase of the spatial-mode state is $\pi$. Here,

$$
\begin{aligned}
\left|\phi^{ \pm}\right\rangle_{P} & =\frac{1}{\sqrt{2}}(|R R\rangle \pm|L L\rangle), \\
\left|\psi^{ \pm}\right\rangle_{P} & =\frac{1}{\sqrt{2}}(|R L\rangle \pm|L R\rangle), \\
\left|\phi^{ \pm}\right\rangle_{S} & =\frac{1}{\sqrt{2}}\left(\left|a_{1} b_{1}\right\rangle \pm\left|a_{2} b_{2}\right\rangle\right), \\
\left|\psi^{ \pm}\right\rangle_{S} & =\frac{1}{\sqrt{2}}\left(\left|a_{1} b_{2}\right\rangle \pm\left|a_{2} b_{1}\right\rangle\right) .
\end{aligned}
$$

If the Hadamard operations are performed on both the spatial-mode and polarization DOFs of photons $A$ and $B$ (as shown in Fig. 21b) before and after they pass through the quantum circuit in Fig. $21 \mathrm{a}$, the result of the polarization-spatial parity-check QND is obtained. That is, if the state of the excess electron spin $e_{1}$ is $|+\rangle_{e_{1}}$, the polarization state is in an even-parity mode. If the state of the excess electron spin $e_{1}$ is $|-\rangle_{e_{1}}$, the polarization state is in an odd-parity mode. If the state of the excess electron spin $e_{2}$ is $|-\rangle_{e_{2}}$, the spatial-mode state is in an even-parity mode. If the state of the excess electron spin $e_{2}$ is $|+\rangle_{e_{2}}$, the spatial-mode state is in an odd-parity mode.

The two-step hyper-EPP for mixed hyperentangled Bell states with polarization bit-flip errors and spatialmode phase-flip errors is constructed with the P-S-QND and the QSJM [54], as shown in Fig. 22.

The first step of the hyper-EPP — The setup of the first step of the hyper-EPP for mixed hyperentangled Bell states is shown in Fig. 222 . Here, the phase-flip errors of Bell states can be transformed to the bit-flip errors by performing Hadamard operations on the two qubits. Suppose that there are two identical nonlocal two-photon systems $A B$ and $C D$, and they are in the states

$$
\begin{aligned}
\rho_{A B}= & {\left[F_{1}\left|\phi^{+}\right\rangle_{P}\left\langle\phi^{+}\left|+\left(1-F_{1}\right)\right| \psi^{+}\right\rangle_{P}\left\langle\psi^{+}\right|\right]_{A B} } \\
& \otimes\left[F_{2}\left|\phi^{+}\right\rangle_{S}\left\langle\phi^{+}\left|+\left(1-F_{2}\right)\right| \phi^{-}\right\rangle_{S}\left\langle\phi^{-}\right|\right]_{A B}, \\
\rho_{C D}= & {\left[F_{1}\left|\phi^{+}\right\rangle_{P}\left\langle\phi^{+}\left|+\left(1-F_{1}\right)\right| \psi^{+}\right\rangle_{P}\left\langle\psi^{+}\right|\right]_{C D} } \\
& \otimes\left[F_{2}\left|\phi^{+}\right\rangle_{S}\left\langle\phi^{+}\left|+\left(1-F_{2}\right)\right| \phi^{-}\right\rangle_{S}\left\langle\phi^{-}\right|\right]_{C D}
\end{aligned}
$$

Here the subscripts $A B$ and $C D$ represent two nonlocal photon pairs. The two photons $A$ and $C$ are obtained by Alice, and the two photons $B$ and $D$ are obtained by Bob. $F_{1}$ and $F_{2}$ represent the probabilities of $\left|\phi^{+}\right\rangle_{P}$ and $\left|\phi^{+}\right\rangle_{S}$ in the mixed state $\rho$, respectively.

Initially, the four-photon system $A B C D$ is in the state $\rho_{0}=\rho_{A B} \otimes \rho_{C D}$, which is a mixed state composed of 16 maximally hyperentangled pure states. Alice and Bob both perform $\mathrm{H}_{P}, \mathrm{P}-\mathrm{S}-\mathrm{QND}$, and $\mathrm{H}_{P S}$ operations on the polarization and spatial-mode DOFs of their photon pairs $A C$ and $B D$. After the measurements are performed on the excess electron spins $e_{1}$ and $e_{2}$ in P-S-QND, the states of the four-photon systems can be divided into four cases, which are discussed in detail as follows.

(1) The results of the P-S-QNDs show that the two photon pairs $A C$ and $B D$ are in the same polarization parity mode and the same spatial-mode parity mode. The polarization state of the four-photon system $A B C D$ is projected to a mixed state that consists of $\left|\Psi_{1}\right\rangle_{P}$ and $\left|\Psi_{2}\right\rangle_{P}\left(\right.$ or $\left|\widetilde{\Psi}_{1}\right\rangle_{P}$ and $\left.\left|\widetilde{\Psi}_{2}\right\rangle_{P}\right)$, and the spatial-mode state of four-photon system $A B C D$ is projected to a mixed state that consists of $\left|\Psi_{1}\right\rangle_{S}$ and $\left|\Psi_{2}\right\rangle_{S}$ (or $\left|\widetilde{\Psi}_{1}\right\rangle_{S}$ and 
$\left.\left|\widetilde{\Psi}_{2}\right\rangle_{S}\right)$. Here

$$
\begin{aligned}
\left|\Psi_{1}\right\rangle_{P} & =\frac{1}{\sqrt{2}}(|R R R R\rangle+|L L L L\rangle), \\
\left|\Psi_{2}\right\rangle_{P} & =\frac{1}{\sqrt{2}}(|R L R L\rangle+|L R L R\rangle), \\
\left|\widetilde{\Psi}_{1}\right\rangle_{P} & =\frac{1}{\sqrt{2}}(|R R L L\rangle+|L L R R\rangle), \\
\left|\widetilde{\Psi}_{2}\right\rangle_{P} & =\frac{1}{\sqrt{2}}(|R L L R\rangle+|L R R L\rangle), \\
\left|\Psi_{1}\right\rangle_{S} & =\frac{1}{\sqrt{2}}\left(\left|a_{1} b_{1} c_{1} d_{1}\right\rangle+\left|a_{2} b_{2} c_{2} d_{2}\right\rangle\right), \\
\left|\Psi_{2}\right\rangle_{S} & =\frac{1}{\sqrt{2}}\left(\left|a_{1} b_{2} c_{1} d_{2}\right\rangle+\left|a_{2} b_{1} c_{2} d_{1}\right\rangle\right), \\
\left|\widetilde{\Psi}_{1}\right\rangle_{S} & =\frac{1}{\sqrt{2}}\left(\left|a_{1} b_{1} c_{2} d_{2}\right\rangle+\left|a_{2} b_{2} c_{1} d_{1}\right\rangle\right), \\
\left|\widetilde{\Psi}_{2}\right\rangle_{S} & =\frac{1}{\sqrt{2}}\left(\left|a_{1} b_{2} c_{2} d_{1}\right\rangle+\left|a_{2} b_{1} c_{1} d_{2}\right\rangle\right) .
\end{aligned}
$$

The state $\left|\widetilde{\Psi}_{i}\right\rangle_{P}\left(\left|\widetilde{\Psi}_{i}\right\rangle_{S}\right)$ can be transformed to the state $\left|\Psi_{i}\right\rangle_{P}\left(\left|\Psi_{i}\right\rangle_{S}\right)$ by performing the polarization (spatialmode) bit-flip operations on photons $C$ and $D$. After Alice and Bob perform the Hadamard operations and detections on the polarization and spatial-mode DOFs of the two photons $C$ and $D$ and the conditional local phase-flip operations $\sigma_{z}^{S}\left(\sigma_{z}^{P}\right)$ on photon $B$, the state of the two-photon system $A B$ is projected to

$$
\begin{aligned}
\rho_{A B}^{\prime}= & {\left[F_{1}^{\prime}\left|\phi^{+}\right\rangle_{P}^{A B}\left\langle\phi^{+}\left|+\left(1-F_{1}^{\prime}\right)\right| \psi^{+}\right\rangle_{P}^{A B}\left\langle\psi^{+}\right|\right] } \\
& \otimes\left[F_{2}^{\prime}\left|\phi^{+}\right\rangle_{S}^{A B}\left\langle\phi^{+}\left|+\left(1-F_{2}^{\prime}\right)\right| \psi^{+}\right\rangle_{S}^{A B}\left\langle\psi^{+}\right|\right],
\end{aligned}
$$

where $F_{i}^{\prime}=\frac{F_{i}^{2}}{F_{i}^{2}+\left(1-F_{i}\right)^{2}}, F_{i}>1 / 2(i=1,2)$.

(2) The results of the P-S-QNDs show that the two photon pairs $A C$ and $B D$ are in the different polarization parity modes and the different spatial-mode parity modes. The polarization state of the four-photon system $A B C D$ is projected to a mixed state that consists of $\left|\Psi_{3}\right\rangle_{P}$ and $\left|\Psi_{4}\right\rangle_{P}$ (or $\left|\widetilde{\Psi}_{3}\right\rangle_{P}$ and $\left|\widetilde{\Psi}_{4}\right\rangle_{P}$ ), and the spatialmode state of the four-photon system $A B C D$ is projected to a mixed state that consists of $\left|\Psi_{3}\right\rangle_{S}$ and $\left|\Psi_{4}\right\rangle_{S}$ (or
$\left|\widetilde{\Psi}_{3}\right\rangle_{S}$ and $\left.\left|\widetilde{\Psi}_{4}\right\rangle_{S}\right)$. Here

$$
\begin{aligned}
&\left|\Psi_{3}\right\rangle_{P}=\frac{1}{\sqrt{2}}(|R R R L\rangle+|L L L R\rangle), \\
&\left|\Psi_{4}\right\rangle_{P}=\frac{1}{\sqrt{2}}(|R L R R\rangle+|L R L L\rangle), \\
&\left|\widetilde{\Psi}_{3}\right\rangle_{P}=\frac{1}{\sqrt{2}}(|R R L R\rangle+|L L R L\rangle), \\
&\left|\widetilde{\Psi}_{4}\right\rangle_{P}=\frac{1}{\sqrt{2}}(|R L L L\rangle+|L R R R\rangle), \\
&\left|\Psi_{3}\right\rangle_{S}=\frac{1}{\sqrt{2}}\left(\left|a_{1} b_{1} c_{1} d_{2}\right\rangle+\left|a_{2} b_{2} c_{2} d_{1}\right\rangle\right), \\
&\left|\Psi_{4}\right\rangle_{S}=\frac{1}{\sqrt{2}}\left(\left|a_{1} b_{2} c_{1} d_{1}\right\rangle+\left|a_{2} b_{1} c_{2} d_{2}\right\rangle\right), \\
&\left|\widetilde{\Psi}_{3}\right\rangle_{S}=\frac{1}{\sqrt{2}}\left(\left|a_{1} b_{1} c_{2} d_{1}\right\rangle+\left|a_{2} b_{2} c_{1} d_{2}\right\rangle\right), \\
&\left|\widetilde{\Psi}_{4}\right\rangle_{S}=\frac{1}{\sqrt{2}}\left(\left|a_{1} b_{2} c_{2} d_{2}\right\rangle+\left|a_{2} b_{1} c_{1} d_{1}\right\rangle\right) .
\end{aligned}
$$

In this case, Alice and Bob cannot distinguish which one of the photon pairs $A B$ and $C D$ has the polarization bitflip error (or the spatial-mode bit-flip error), so the two photon pairs have to be discarded.

(3) The results of the P-S-QNDs show that the two photon pairs $A C$ and $B D$ are in the same polarization parity mode and the different spatial-mode parity modes. The polarization state of the four-photon system $A B C D$ is projected to a mixed state that consists of $\left|\Psi_{1}\right\rangle_{P}$ and $\left|\Psi_{2}\right\rangle_{P}$ (or $\left|\widetilde{\Psi}_{1}\right\rangle_{P}$ and $\left|\widetilde{\Psi}_{2}\right\rangle_{P}$ ), and the spatial-mode state of the four-photon system $A B C D$ is projected to a mixed state that consists of $\left|\Psi_{3}\right\rangle_{S}$ and $\left|\Psi_{4}\right\rangle_{S}$ (or $\left|\widetilde{\Psi}_{3}\right\rangle_{S}$ and $\left.\left|\widetilde{\Psi}_{4}\right\rangle_{S}\right)$. After Alice and Bob perform the Hadamard operations and detections on the polarization and spatialmode DOFs of the two photons $C$ and $D$ and the conditional local phase-flip operations $\sigma_{z}^{S}\left(\sigma_{z}^{P}\right)$ on photon $B$, the state of the two-photon system $A B$ is projected to

$$
\begin{aligned}
\rho_{A B}^{\prime \prime}= & {\left[F_{1}^{\prime}\left|\phi^{+}\right\rangle_{P}^{A B}\left\langle\phi^{+}\left|+\left(1-F_{1}^{\prime}\right)\right| \psi^{+}\right\rangle_{P}^{A B}\left\langle\psi^{+}\right|\right] } \\
& \otimes\left[F_{2}^{\prime \prime}\left|\phi^{+}\right\rangle_{S}^{A B}\left\langle\phi^{+}\left|+\left(1-F_{2}^{\prime \prime}\right)\right| \psi^{+}\right\rangle_{S}^{A B}\left\langle\psi^{+}\right|\right],
\end{aligned}
$$

where $F_{i}^{\prime \prime}=\frac{F_{i}\left(1-F_{i}\right)}{2 F_{i}\left(1-F_{i}\right)}$. In this case, Alice and Bob cannot distinguish which one of the photon pairs $A B$ and $C D$ has the spatial-mode bit-flip error, so the second step of hyper-EPP is required to perform on the photon pair $A B$ to obtain the high-fidelity hyperentangled state.

(4) The results of the P-S-QNDs show that the two photon pairs $A C$ and $B D$ are in the different polarization parity modes and the same spatial-mode parity mode. The polarization state of the four-photon system $A B C D$ is projected to a mixed state that consists of $\left|\Psi_{3}\right\rangle_{P}$ and $\left|\Psi_{4}\right\rangle_{P}$ (or $\left|\widetilde{\Psi}_{3}\right\rangle_{P}$ and $\left|\widetilde{\Psi}_{4}\right\rangle_{P}$ ), and the spatial-mode state of the four-photon system $A B C D$ is projected to a mixed state that consists of $\left|\Psi_{1}\right\rangle_{S}$ and $\left|\Psi_{2}\right\rangle_{S}$ (or $\left|\widetilde{\Psi}_{1}\right\rangle_{S}$ and $\left.\left|\widetilde{\Psi}_{2}\right\rangle_{S}\right)$. After Alice and Bob perform the Hadamard operations and detections on the polarization and spatialmode DOFs of the two photons $C$ and $D$ and the condi- 
tional local phase-flip operations $\sigma_{z}^{S}\left(\sigma_{z}^{P}\right)$ on photon $B$, the state of the two-photon system $A B$ is projected to

$$
\begin{aligned}
\rho_{A B}^{\prime \prime \prime}= & {\left[F_{1}^{\prime \prime}\left|\phi^{+}\right\rangle_{P}^{A B}\left\langle\phi^{+}\left|+\left(1-F_{1}^{\prime \prime}\right)\right| \psi^{+}\right\rangle_{P}^{A B}\left\langle\psi^{+}\right|\right] } \\
& \otimes\left[F_{2}^{\prime}\left|\phi^{+}\right\rangle_{S}^{A B}\left\langle\phi^{+}\left|+\left(1-F_{2}^{\prime}\right)\right| \psi^{+}\right\rangle_{S}^{A B}\left\langle\psi^{+}\right|\right] .
\end{aligned}
$$

In this case, Alice and Bob cannot distinguish which one of the photon pairs $A B$ and $C D$ has the polarization bitflip error, so the second step of hyper-EPP is required to perform on the photon pair $A B$ to obtain the high-fidelity hyperentangled state.

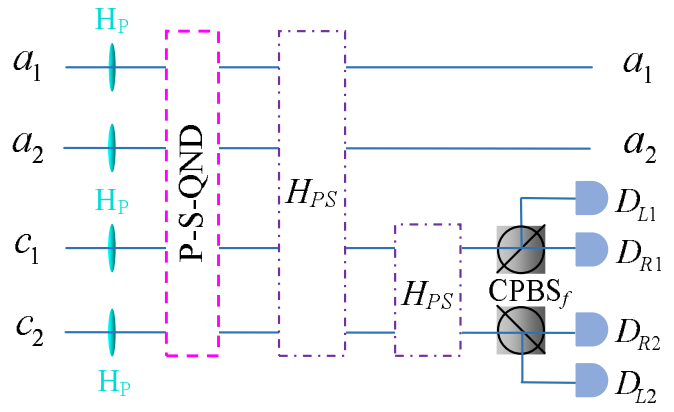

(a)

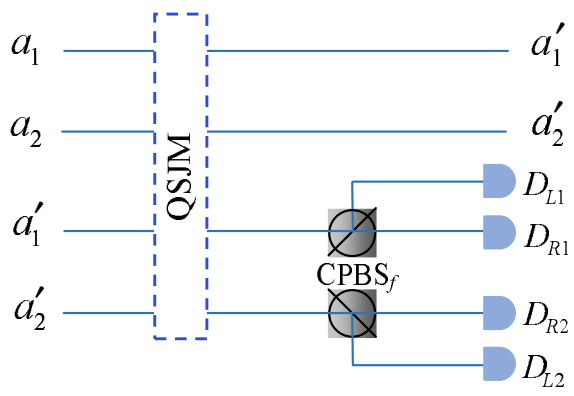

(b)

FIG. 22: (a) Schematic diagram of the first step of the twostep hyper-EPP with the P-S-QND [54]. The operations performed on the photons $B$ and $D$ are the same as the ones performed on the photons $A$ and $C$. (b) Schematic diagram of the second step of the two-step hyper-EPP with QSJM. The operations performed on the photons $B$ and $B^{\prime}$ are the same as the ones performed on the photons $A$ and $A^{\prime}$.

The second step of the hyper-EPP - Here, four identical nonlocal photon pairs $A B, C D, A^{\prime} B^{\prime}$, and $C^{\prime} D^{\prime}$ are required. The photons $A, C, A^{\prime}$, and $C^{\prime}$ are obtained by Alice, and the photons $B, D, B^{\prime}$, and $D^{\prime}$ are obtained by Bob. In the first step, the same operations are performed on the four-photon systems $A B C D$ and $A^{\prime} B^{\prime} C^{\prime} D^{\prime}$. If the results of the P-S-QNDs showed that the four-photon systems $A B C D$ and $A^{\prime} B^{\prime} C^{\prime} D^{\prime}$ are projected to the cases (3) and (4) in the first step, respectively, the QSJM is introduced to combine the polarization state of the photon pair $A B$ and the spatial-mode state of the photon pair $A^{\prime} B^{\prime}$ into an output single photon-pair state [54]. So the preserving condition of the case (1) in the first step is achieved.

If the results of the P-S-QNDs showed that the fourphoton systems $A B C D$ and $A^{\prime} B^{\prime} C^{\prime} D^{\prime}$ are projected to the cases (4) and (3) in the first step, respectively, the QSJM is introduced to combine the spatial-mode state of the photon pair $A B$ and the polarization state of the photon pair $A^{\prime} B^{\prime}$ into an output single photon-pair state. So the preserving condition of the case (1) in the first step is also achieved.

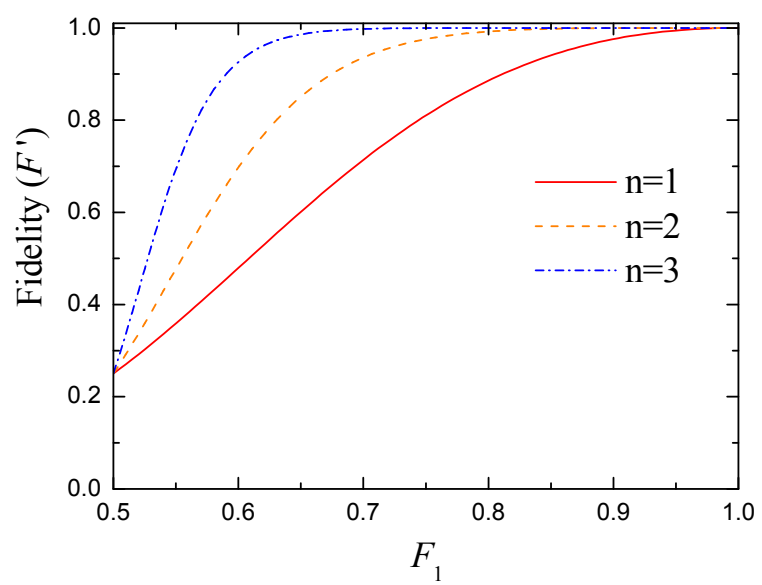

FIG. 23: The fidelity of the hyperentangled Bell state obtained in the hyper-EPP versus the fidelity of the initial mixed hyperentangled Bell state $\left(F_{1}\right)$ under the iteration number $(n)$ [54]. Here, the parameters of the initial mixed hyperentangled Bell state are $F_{1}=F_{2}$.

Fidelity and efficiency - After the two steps of hyperEPP are performed on the nonlocal photon systems, the final state of the photon pair $A B$ is obtained as

$$
\begin{aligned}
\rho_{A B}^{\prime}= & {\left[F_{1}^{\prime}\left|\phi^{+}\right\rangle_{A B}^{P}\left\langle\phi^{+}\left|+\left(1-F_{1}^{\prime}\right)\right| \psi^{+}\right\rangle_{A B}^{P}\left\langle\psi^{+}\right|\right] } \\
& \otimes\left[F_{2}^{\prime}\left|\phi^{+}\right\rangle_{A B}^{S}\left\langle\phi^{+}\left|+\left(1-F_{2}^{\prime}\right)\right| \psi^{+}\right\rangle_{A B}^{S}\left\langle\psi^{+}\right|\right] .
\end{aligned}
$$

The fidelity of the state $\left|\phi^{+}\right\rangle_{A B}^{P} \otimes\left|\phi^{+}\right\rangle_{A B}^{S}$ in $\rho_{A B}^{\prime}$ is increased from $F=F_{1} \times F_{2}$ to $F^{\prime}=F_{1}^{\prime} \times F_{2}^{\prime}$. By performing the Hadamard operations on the spatial-mode DOF of the photons $A$ and $B$, the state $\left|\psi^{+}\right\rangle_{A B}^{S}$ can be transformed to $\left|\phi^{-}\right\rangle_{A B}^{S}$. The fidelity of this two-photon hyperentangled state can be greatly improved by iterative application of the two-step hyper-EPP process, as shown in Fig. 23.

The efficiency is defined as the probability to obtain a high-fidelity entangled photon system from a pair of lessentangled photon systems after they transmitted over a noisy channel (without considering the photon loss). In 


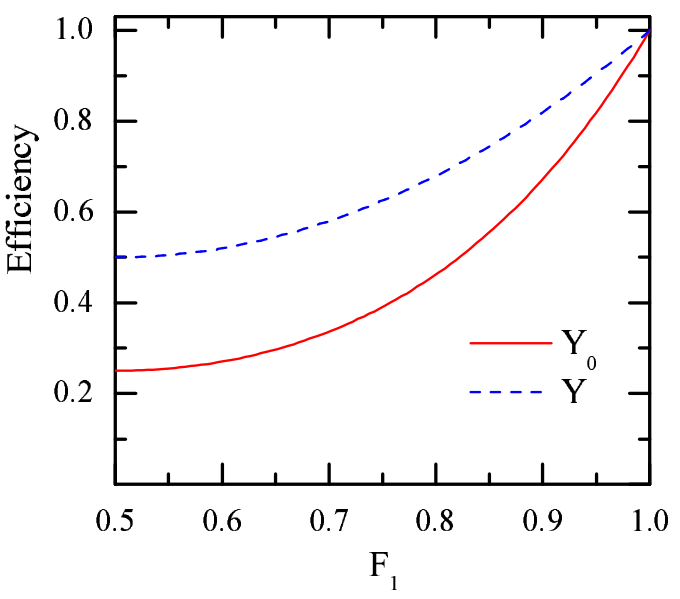

FIG. 24: The efficiency of the two-step hyper-EPP for mixed hyperentangled Bell states [54]. $Y_{0}(Y)$ is the efficiency of the first round of the hyper-EPP process without (with) QSJM. The parameters of the initial mixed hyperentangled Bell state are $F_{1}=F_{2}$.

the hyper-EPP without QSJM, only the case (1) in the first step is preserved, so the efficiency of the first round in the hyper-EPP process is

$$
Y_{0}=\left[F_{1}^{2}+\left(1-F_{1}\right)^{2}\right] \times\left[F_{2}^{2}+\left(1-F_{2}\right)^{2}\right] .
$$

In the two-step hyper-EPP with QSJM, the high-fidelity hyperentangled states can also be obtained from the cases (3) and (4) in the first step, so the efficiency of the first round in the hyper-EPP process is

$$
Y=F_{2}^{2}+\left(1-F_{2}\right)^{2}
$$

Here $F_{1}>F_{2}$. Now, we can see that the efficiency of the hyper-EPP is greatly improved by introducing the second step with QSJM, as shown in Fig. $24\left(F_{1}=F_{2}\right)$.

\section{HYPERPARALLEL PHOTONIC QUANTUM COMPUTATION}

Photonic quantum computation is an important branch of parallel quantum computation. With the nonlinear interaction between a photon and an artificial atomic system, photonic quantum gates are scalable as the same as the universal quantum gates on solid-state quantum systems, such as circuit quantum electrodynamics (QED) with superconducting Josephson junctions (act as the artificial atoms) and a superconducting resonator (acts as a cavity and quantum bus) [107-112], diamond nitrogen-vacancy center 113 116], quantum dots [117 122], nuclear magnetic resonance [123 126], and cavity QED [127, 128], especially with the development of the manipulation of single photons [129, 130]. Moreover, a photon system has multiple DOFs which can be used to encode information in quantum computation [131], such as polarization, spatial-mode, frequency, orbital angular momentum, transverse, energy-time, time bin, and so on. With the polarization DOF of photon systems, many quantum logic gates have been constructed either in theory or in experiment 132 140]. The quantum logic gates and the quantum algorithms on a photon with two DOFs have also been investigated in the past few years 141 149], although their scalability is not good with linear or nonlinear optical elements.

In 2013, the concept of hyperparallel photonic quantum computation, performing universal quantum gate operations on two-photon or multi-photon systems by encoding all the quantum states of each photon in multiple DOFs (two or more DOFs) as information carriers, was introduced by Ren et al. [58]. They proposed the first scheme for the hyper-CNOT gate operating on both the spatial-mode and the polarization DOFs of a twophoton system simultaneously. In this scheme, both the polarization quantum state and the spatial-mode quantum state of each photon are encoded as the qubits for carrying information, not exploiting one DOF to implement a CNOT gate on the other DOF of a single photon, far different from conventional parallel quantum computation. In 2014, Ren and Deng 59] proposed another scheme for hyperparallel photonic quantum computation assisted by the giant optical circular birefringence induced by quantum-dot spins in double-sided optical microcavities. It has a simpler quantum circuit. In 2015, Ren et al. [60] designed two universal hyperparallel hybrid photonic quantum logic gates with dipole-induced transparency of a diamond NV center embedded in a photonic crystal cavity coupled to two waveguides in the weak-coupling regime, including a hybrid hyper-CNOT gate and a hybrid hyper-Toffoli gate on photon systems in both the polarization and the spatial-mode DOFs, which are equal to two identical quantum logic gates operating simultaneously on the two-photon systems in one DOF. Now, some important schemes for the hyperparallel photonic quantum computation are proposed, including hyperparallel two-photon gates [58 61] and hyperparallel three-photon gates (hyperparallel Toffoli gates and Fredkin gates) 60, 62]. With hyperparallel photonic quantum logic gates, the resource consumed can be reduced and the photonic dispassion noise can be depressed in quantum circuit [60]. Moreover, the multiple-photon hyperentangled state can be prepared and measured with less resource and less steps by using the hyperparallel photonic quantum logic gates, which may speedup the quantum algorithm [58, 59].

Here, we introduce the principle of hyperparallel photonic quantum computation by describing the process for constructing the hyper-CNOT gate [59] on both the polarization and spatial-mode DOFs of a two-photon system, assisted by the nonlinearity of double-sided QDcavity systems. This gate can achieve scalable hyperparallel quantum computation without using auxiliary spatial modes or polarization modes.

The setup of the hyper-CNOT gate can also be described by Fig. 21]. The states of the excess electron spins $e_{1}$ in $\mathrm{QD}_{1}$ and $e_{2}$ in $\mathrm{QD}_{2}$ are prepared in $|+\rangle_{e_{1}}$ and 
$|+\rangle_{e_{2}}$, respectively. The initial states of the two photons $A$ and $B$ are $\left|\varphi_{A}\right\rangle_{0}=\left(\alpha_{1}|R\rangle+\alpha_{2}|L\rangle\right)_{A}\left(\gamma_{1}\left|a_{1}\right\rangle+\gamma_{2}\left|a_{2}\right\rangle\right)$ and $\left|\varphi_{B}\right\rangle_{0}=\left(\beta_{1}|R\rangle+\beta_{2}|L\rangle\right)_{B}\left(\delta_{1}\left|b_{1}\right\rangle+\delta_{2}\left|b_{2}\right\rangle\right)$, respectively.

First, the Hadamard operations are performed on the polarization and spatial-mode DOFs of photon $A$, and the state of photon $A$ is transformed to $\left|\varphi_{A}^{\prime}\right\rangle_{0}=\left(\alpha_{1}^{\prime}|R\rangle+\right.$ $\left.\alpha_{2}^{\prime}|L\rangle\right)_{A}\left(\gamma_{1}^{\prime}\left|a_{1}\right\rangle+\gamma_{2}^{\prime}\left|a_{2}\right\rangle\right)$. Then the two spatial modes $a_{1}$ and $a_{2}$ of photon $A$ are put into $\mathrm{CPBS}_{1}, \mathrm{U}_{1}, \mathrm{QD}_{1}, \mathrm{U}_{2}$, and $\mathrm{CPBS}_{2}$ in sequence as shown in Fig. 21 a, and the state of the system $A e_{1}$ is transformed from $\left|\varphi_{A e_{1}}\right\rangle_{0}$ to $\left|\varphi_{A e_{1}}\right\rangle_{1}$. Here

$$
\begin{aligned}
\left|\varphi_{A e_{1}}\right\rangle_{0}= & \frac{1}{\sqrt{2}}(|\uparrow\rangle+|\downarrow\rangle)_{e_{1}} \otimes \\
& \left(\alpha_{1}^{\prime}|R\rangle+\alpha_{2}^{\prime}|L\rangle\right)_{A}\left(\gamma_{1}^{\prime}\left|a_{1}\right\rangle+\gamma_{2}^{\prime}\left|a_{2}\right\rangle\right), \\
\left|\varphi_{A e_{1}}\right\rangle_{1}= & \frac{1}{\sqrt{2}}\left\{\gamma _ { 1 } ^ { \prime } \left[|\uparrow\rangle_{e_{1}}\left(\alpha_{1}^{\prime}|L\rangle+\alpha_{2}^{\prime}|R\rangle\right)_{A}\right.\right. \\
& \left.\left.+|\downarrow\rangle_{e_{1}}\left(\alpha_{1}^{\prime}|R\rangle+\alpha_{2}^{\prime}|L\rangle\right)_{a}\right)\right]\left|a_{2}\right\rangle \\
& +\gamma_{2}^{\prime}\left[|\uparrow\rangle_{e_{1}}\left(\alpha_{1}^{\prime}|R\rangle+\alpha_{2}^{\prime}|L\rangle\right)_{A}\right. \\
& \left.\left.\left.+|\downarrow\rangle_{e_{1}}\left(\alpha_{1}^{\prime}|L\rangle+\alpha_{2}^{\prime}|R\rangle\right)_{a}\right)\right]\left|a_{1}\right\rangle\right\}
\end{aligned}
$$

After the two spatial modes $a_{1}$ and $a_{2}$ pass through $\mathrm{CPBS}_{2}$, we put them into $\mathrm{QD}_{2}, \mathrm{X}, \mathrm{CPBS}_{3}, \mathrm{Z}_{1}$, and $\mathrm{Z}_{2}$ in sequence as shown in Fig. 21 a, and the state of the system $A e_{1} e_{2}$ is transformed from $\left|\varphi_{A e_{1} e_{2}}\right\rangle_{1}$ to $\left|\varphi_{A e_{1} e_{2}}\right\rangle_{2}$. Here

$$
\begin{aligned}
\left|\varphi_{A e_{1} e_{2}}\right\rangle_{1}= & \frac{1}{\sqrt{2}}(|\uparrow\rangle+|\downarrow\rangle)_{e_{2}} \otimes\left|\varphi_{A e_{1}}\right\rangle_{1}, \\
\left|\varphi_{A e_{1} e_{2}}\right\rangle_{2}= & \frac{1}{2}\left[|\uparrow\rangle_{e_{1}}\left(\alpha_{1}^{\prime}|R\rangle+\alpha_{2}^{\prime}|L\rangle\right)_{A}+|\downarrow\rangle_{e_{1}}\left(\alpha_{2}^{\prime}|R\rangle\right.\right. \\
& \left.\left.\left.+\alpha_{1}^{\prime}|L\rangle\right)_{A}\right)\right]\left[|\uparrow\rangle_{e_{2}}\left(\gamma_{2}^{\prime}\left|a_{1}\right\rangle+\gamma_{1}^{\prime}\left|a_{2}\right\rangle\right)\right. \\
& \left.-|\downarrow\rangle_{e_{2}}\left(\gamma_{1}^{\prime}\left|a_{1}\right\rangle+\gamma_{2}^{\prime}\left|a_{2}\right\rangle\right)\right] .
\end{aligned}
$$

Now, we have obtained the result of the four-qubit hybrid CNOT gate.

Subsequently, we perform the Hadamard operations on the electron spins $e_{1}$ and $e_{2}$, and we put the two spatial modes $b_{1}$ and $b_{2}$ of photon $B$ into $\mathrm{CPBS}_{1}, \mathrm{U}_{1}, \mathrm{QD}_{1}$, $\mathrm{U}_{2}, \mathrm{CPBS}_{2}, \mathrm{QD}_{2}, \mathrm{X}, \mathrm{CPBS}_{3}, \mathrm{Z}_{1}$, and $\mathrm{Z}_{2}$ in sequence as shown in Fig. 21a. Then the state of the system $A B e_{1} e_{2}$ is transformed from $\left|\varphi_{A B e_{1} e_{2}}\right\rangle_{2}$ to $\left|\varphi_{A B e_{1} e_{2}}\right\rangle_{3}$. Here

$$
\begin{aligned}
\left|\varphi_{A B e_{1} e_{2}}\right\rangle_{2}= & \left|\varphi_{A e_{1} e_{2}}\right\rangle_{2}\left(\beta_{1}|R\rangle+\beta_{2}|L\rangle\right)_{B}\left(\delta_{1}\left|b_{1}\right\rangle+\delta_{2}\left|b_{2}\right\rangle\right), \\
\left|\varphi_{A B e_{1} e_{2}}\right\rangle_{3}= & \frac{1}{2}\left[|\uparrow\rangle_{e_{1}} \alpha_{1}(|R\rangle+|L\rangle)_{A}\left(\beta_{1}|R\rangle+\beta_{2}|L\rangle\right)_{B}\right. \\
& \left.+|\downarrow\rangle_{e_{1}} \alpha_{2}(|R\rangle-|L\rangle)_{A}\left(\beta_{2}|R\rangle+\beta_{1}|L\rangle\right)_{B}\right] \\
& \otimes\left[-|\uparrow\rangle_{e_{2}} \gamma_{2}\left(\left|a_{1}\right\rangle-\left|a_{2}\right\rangle\right)\left(\delta_{2}\left|b_{1}\right\rangle+\delta_{1}\left|b_{2}\right\rangle\right)\right. \\
& \left.+|\downarrow\rangle_{e_{2}} \gamma_{1}\left(\left|a_{1}\right\rangle+\left|a_{2}\right\rangle\right)\left(\delta_{1}\left|b_{1}\right\rangle+\delta_{2}\left|b_{2}\right\rangle\right)\right] . \quad(73)
\end{aligned}
$$

Finally, the Hadamard operations are performed on the spatial-mode and polarization DOFs of photon $A$ and the excess electron spins $e_{1}$ and $e_{2}$ again, and the state of the system $A B e_{1} e_{2}$ is changed to

$$
\begin{aligned}
\left|\varphi_{A B e_{1} e_{2}}\right\rangle_{4}= & \frac{1}{2}\left\{| \uparrow \rangle _ { e _ { 1 } } \left[\alpha_{1}|R\rangle_{A}\left(\beta_{1}|R\rangle+\beta_{2}|L\rangle\right)_{B}\right.\right. \\
& \left.+\alpha_{2}|L\rangle_{A}\left(\beta_{2}|R\rangle+\beta_{1}|L\rangle\right)_{B}\right] \\
& +|\downarrow\rangle_{e_{1}}\left[\alpha_{1}|R\rangle_{A}\left(\beta_{1}|R\rangle+\beta_{2}|L\rangle\right)_{B}\right. \\
& \left.\left.-\alpha_{2}|L\rangle_{A}\left(\beta_{2}|R\rangle+\beta_{1}|L\rangle\right)_{B}\right]\right\} \\
& \otimes\left\{| \uparrow \rangle _ { e _ { 2 } } \left[\gamma_{1}\left|a_{1}\right\rangle\left(\delta_{1}\left|b_{1}\right\rangle+\delta_{2}\left|b_{2}\right\rangle\right)\right.\right. \\
& \left.-\gamma_{2}\left|a_{2}\right\rangle\left(\delta_{2}\left|b_{1}\right\rangle+\delta_{1}\left|b_{2}\right\rangle\right)\right] \\
& -|\downarrow\rangle_{e_{2}}\left[\gamma_{1}\left|a_{1}\right\rangle\left(\delta_{1}\left|b_{1}\right\rangle+\delta_{2}\left|b_{2}\right\rangle\right)\right. \\
& \left.\left.+\gamma_{2}\left|a_{2}\right\rangle\left(\delta_{2}\left|b_{1}\right\rangle+\delta_{1}\left|b_{2}\right\rangle\right)\right]\right\} .
\end{aligned}
$$

Then the two excess electron spins $e_{1}$ and $e_{2}$ are measured in the orthogonal basis $\left\{|\uparrow\rangle_{e},|\downarrow\rangle_{e}\right\}$. If the state of electron spin $e_{1}$ is $|\downarrow\rangle_{e_{1}}$, an additional sign change $|L\rangle_{A} \rightarrow-|L\rangle_{A}$ is performed on photon $A$. If the state of electron spin $e_{2}$ is $|\uparrow\rangle_{e_{2}}$, an additional sign change $\left|a_{2}\right\rangle \rightarrow-\left|a_{2}\right\rangle$ is performed on photon $A$. Now, we can obtain the result of the spatial-polarization hyper-CNOT gate,

$$
\begin{aligned}
\left|\varphi_{A B}\right\rangle=[ & \alpha_{1}|R\rangle_{A}\left(\beta_{1}|R\rangle+\beta_{2}|L\rangle\right)_{B}+\alpha_{2}|L\rangle_{A}\left(\beta_{2}|R\rangle\right. \\
& \left.\left.+\beta_{1}|L\rangle\right)_{B}\right] \otimes\left[\gamma_{1}\left|a_{1}\right\rangle\left(\delta_{1}\left|b_{1}\right\rangle+\delta_{2}\left|b_{2}\right\rangle\right)\right. \\
& \left.+\gamma_{2}\left|a_{2}\right\rangle\left(\delta_{2}\left|b_{1}\right\rangle+\delta_{1}\left|b_{2}\right\rangle\right)\right] .
\end{aligned}
$$

Here the polarization and spatial-mode DOFs of photon $A$ are used as control qubits and the polarization and spatial-mode DOFs of photon $B$ are used as target qubits.

\section{DISCUSSION AND SUMMARY}

In this review, we have introduced the preparation of hyperentanglement and its application in QIP. Hyperentanglement is defined as the entanglement in multiple DOFs of photon system, and it can be prepared with the combination of the techniques used for creating the entanglement in a single DOF [18 25]. In quantum communication, hyperentanglement can be used to increase the channel capacity largely, besides its application for assisting the implementation of quantum communication protocols based on one DOF. HBSA is the prerequisite for quantum communication protocols with hyperentanglement, and it is one of the important parts in high-capacity quantum repeaters [36-45]. We have reviewed the high-capacity long-distance quantum communication protocols based on polarization-spatial hyperentanglement, including the complete HBSA scheme with the cross-Kerr nonlinearity, the quantum teleportation of a quantum state in both the polarization and the spatial-mode DOFs with polarization-spatial hyperentanglement, and the hyperentanglement swapping of polarization-spatial hyperentangled Bell states. They are useful tools in high-capacity quantum communication protocols and high-capacity quantum repeaters. 
In practical applications, the maximally entangled photon systems are produced locally, which leads to the decoherence of the photon systems by the environment noise in their distribution and storage processes in QIP. To depress this decoherence, entanglement concentration [46 53] and entanglement purification [47, 5457], two passive ways for nonlocal quantum systems to overcome the adverse influence from noise, were introduced for long-distance quantum communication assisted by quantum repeaters [5 13, 150, 151]. Hyper-ECPs are used to obtain the maximally hyperentangled states from nonlocal partially hyperentangled pure states. The hyper-ECPs can be implemented with two methods: the parameter-splitting method [46] and the Schmidt projection method [46, 47], which are useful for improving the entanglement of the partially hyperentangled states with their parameters known and unknown to the remote users, respectively. In a practical quantum communication, the information about the parameters of a nonlocal partially hyperentangled pure state can be obtained by measuring an enough number of sample photon pairs, and the parameter-splitting method is far more efficient than the Schmidt projection method when they are used to obtain maximally entangled states in the case with a large number of quantum data needed to be exchanged between the two parties. While, if there are a small quantity of quantum data needed to be exchanged between the two parties, the hyper-ECPs with the Schmidt projection method may be more practical as they do not require the two parties to measure the samples for obtaining the accurate information about the parameters of the partially hyperentangled pure state. In contrast with hyper-ECPs, hyper-EPPs are more general but they work with a relatively low efficiency. In principle, hyperEPPs are used to obtain the high-fidelity hyperentangled states from nonlocal mixed hyperentangled states with less entanglement, not a pure state. As an example, we introduced the two-step hyper-EPP for mixed hyperentangled Bell states with both the polarization bit-flip errors and the spatial-mode phase-flip errors, resorting to the nonlinearity of double-sided QD-cavity system. The P-S-QND and QSJM are two basic quantum gate operations for the two steps, respectively. By introducing the QSJM, the efficiency of the hyper-EPP can be improved by preserving the states that are discarded in the hyperEPP without QSJM. The fidelity of the two-photon state in hyper-EPP can be improved by iterative application of the hyper-EPP process. This hyper-EPP can be generalized to purify the mixed hyperentangled GHZ states with channel errors in both the two DOFs of photon systems.

As another important application of hyperentanglement, we introduced a photonic hyper-CNOT gate for the hyperparallel quantum computation. The hyperparallel quantum logic gate can be used to perform multiple quantum logic gate operations on a photon system compared with those on multiple photon systems $[58$ 62. Recently, the hyperparallel photonic Toffoli gate for a three-photon system has also been proposed [60], which can perform double Toffoli gate operations on a three-photon system. With the hyperparallel quantum logic gate, the hyperentangled states of multiple-photon system can be prepared and analyzed with less resource and steps, which may reduce the resource and steps required for quantum algorithms. Moreover, the hyperparallel quantum logic gates can be used to implement the universal hyperparallel quantum computation tasks on multiple DOFs of photon systems, together with the single photon manipulation.

Now, more and more attention is focused on hyperentanglement and its applications in QIP. Maybe it is also the important resource for other tasks in quantum physics and quantum techniques. Also, it may be interesting to investigate hyperentanglement in relativistic systems [152]. The continuous-variable hyperentanglement of polarization and orbital angular momentum has been generated experimentally with a type-II optical parametric oscillator [153].

\section{Acknowledgment}

This work was supported by the National Natural Science Foundation of China (11474026, 11574038, 11547106, 11604226, and 11674033).

\section{Conflict of interests}

The authors declare that they have no conflict of interests.
[1] Nielsen MA, Chuang LL. Quantum Computation and Quantum Information. Cambridge: Cambridge University Press; 2000.

[2] Galindo A, Martin-Delgado MA. Information and computation: classical and quantum aspects. Rev Mod Phys 2002;74:347-423.

[3] Horodecki R, Horodeki P, Horodecki M, et al. Quantum entanglement. Rev Mod Phys 2009;81:865-942.

[4] Briegel HJ, Dür W, Cirac JI, et al. Quantum repeaters: the role of imperfect local operations in quantum communication. Phys Rev Lett 1998;81:5932-35.

[5] Bennett CH, Brassard G. Quantum cryptography: public key distribution and coin tossing. in Proceedings of IEEE international conference on computers, systems and signal Processing, Bangalore, India (IEEE, New York) (1984) 175-9.

[6] Ekert AK. Quantum cryptography based on Bell's theorem. Phys Rev Lett 1991;67:661-3. 
[7] Bennett CH, Brassard G, Mermin ND. Quantum cryptography without Bell's theorem. Phys Rev Lett 1992;68:557-9.

[8] Hillery M, Bužek V, Berthiaume A. Quantum secret sharing. Phys Rev A 1999;59:1829-34.

[9] Long GL, Liu XS. Theoretically efficient highcapacity quantum-key-distribution scheme. Phys Rev A 2002;65:032302.

[10] Deng FG, Long GL, Liu XS. Two-step quantum direct communication protocol using the Einstein-PodolskyRosen pair block. Phys Rev A 2003;68:042317.

[11] Deng FG, Long GL. Secure direct communication with a quantum one-time pad. Phys Rev A 2004;69:052319.

[12] Hu JY, Yu B, Jing MY, et al. Experimental quantum secure direct communication with single photons. Light Sci Appl 2016;5:e16144.

[13] Zhang W, Ding DS, Sheng YB, et al. Quantum secure direct communication with quantum memory. arXiv:1609.09184.

[14] Kiess TE, Shih YH, Sergienko AV, et al. EinsteinPodolsky-Rosen-Bohm experiment using pairs of light quanta produced by type II parametric down conversion. Phys Rev Lett 1993;71:3893.

[15] Kwiat PG, Waks E, White AG, et al. Ultrabright source of polarization-entangled photons. Phys Rev A 1999;60:R773-6.

[16] Barbieri M, De Martini F, Di Nepi G, et al. Generation and characterization of Werner states and maximally entangled mixed states by a universal source of entanglement. Phys Rev Lett 2004;92:177901.

[17] Kwiat PG. Hyper-entangled states. J Mod Opt 1997;44:2173-84.

[18] Yang T, Zhang Q, Zhang J, et al. All-versus-nothing violation of local realism by two-photon, four-dimensional entanglement. Phys Rev Lett 2005;95:240406.

[19] Barreiro JT, Langford NK, Peters NA, et al. Generation of hyperentangled photon pairs. Phys Rev Lett 2005;95:260501.

[20] Vallone G, Ceccarelli R, De Martini F, et al. Hyperentanglement of two photons in three degrees of freedom. Phys Rev A 2009;79:030301R.

[21] Ceccarelli R, Vallone G, De Martini F, et al. Experimental entanglement and nonlocality of a two-photon six-qubit cluster state. Phys Rev Lett 2009;103:160401.

[22] Vallone G, Donati G, Ceccarelli R, et al. Six-qubit twophoton hyperentangled cluster states: characterization and application to quantum computation. Phys Rev A 2010;81:052301.

[23] Gao WB, Lu CY, Yao XC, et al. Experimental demonstration of a hyper-entangled ten-qubit Schröinger cat state. Nat Phys 2010;6:331-5.

[24] Kang DP, Helt LG, Zhukovsky SV, et al. Hyperentangled photon sources in semiconductor waveguides. Phys Rev A 2014;89:023833.

[25] Hu BL, Zhan YB. Generation of hyperentangled states between remote noninteracting atomic ions. Phys Rev A 2010;82:054301.

[26] Barreiro JT, Wei TC, Kwiat PG. Beating the channel capacity limit for linear photonic superdense coding. Nat Phys 2008;4:282-6.

[27] Kwiat PG, Weinfurter H. Embedded Bell-state analysis. Phys Rev A 1998;58:R2623-6.

[28] Walborn SP, Ṕadua S, Monken CH. Hyperentanglement-assisted Bell-state analysis. Phys
Rev A 2003;68:042313.

[29] Schuck C, Huber G, Kurtsiefer C, et al. Complete deterministic linear optics Bell state analysis. Phys Rev Lett 2006;96:190501.

[30] Barbieri M, Vallone G, Mataloni P, et al. Complete and deterministic discrimination of polarization Bell states assisted by momentum entanglement. Phys Rev A 2007;75:042317

[31] Sheng YB, Deng FG. Deterministic entanglement purification and complete nonlocal Bell-state analysis with hyperentanglement. Phys Rev A 2010;81:032307.

[32] Sheng YB, Deng FG. One-step deterministic polarization-entanglement purification using spatial entanglement. Phys Rev A 2010;82:044305.

[33] Li XH. Deterministic polarization-entanglement purification using spatial entanglement. Phys Rev A 2010;82:044304.

[34] Deng FG. One-step error correction for multipartite polarization entanglement. Phys Rev A 2011;83:062316.

[35] Wang TJ, Song SY, Long GL. Quantum repeater based on spatial entanglement of photons and quantumdot spins in optical microcavities. Phys Rev A 2012;85:062311.

[36] Sheng YB, Deng FG, Long GL. Complete hyperentangled-Bell-state analysis for quantum communication. Phys Rev A 2010;82:032318.

[37] Ren BC, Wei HR, Hua M, et al. Complete hyperentangled-Bell-state analysis for photon systems assisted by quantum-dot spins in optical microcavities. Opt Express 2012;20:24664-77.

[38] Wang TJ, Lu Y, Long GL. Generation and complete analysis of the hyperentangled Bell state for photons assisted by quantum-dot spins in optical microcavities. Phys Rev A 2012;86:042337.

[39] Liu Q, Zhang M. Generation and complete nondestructive analysis of hyperentanglement assisted by nitrogen-vacancy centers in resonators. Phys Rev A 2015;91:062321.

[40] Liu Q, Wang GY, Ai Q, et al. Complete nondestructive analysis of two-photon six-qubit hyperentangled Bell states assisted by cross-Kerr nonlinearity. Sci Rep 2016;6:22016.

[41] Li XH, Ghose S. Self-assisted complete maximally hyperentangled state analysis via the cross-Kerr nonlinearity. Phys Rev A 2016;93:022302.

[42] Li XH, Ghose S. Complete hyperentangled Bell state analysis for polarization and time-bin hyperentanglement. Opt Express 2016;24:18388-98.

[43] Wang TJ, Wang C. Complete hyperentangled-Bell-state analysis for photonic qubits assisted by a three-level Lambda-type system. Sci Rep 2016;6:19497.

[44] Wei TC, Barreiro JT, Kwiat PG. Hyperentangled Bellstate analysis. Phys Rev A 2007:75:060305(R).

[45] Xia Y, Chen QQ, Song J, et al. Efficient hyperentangled Greenberger-Horne-Zeilinger states analysis with crossKerr nonlinearity. J Opt Soc Am B 2012;29:1029-37.

[46] Ren BC, Du FF, Deng FG. Hyperentanglement concentration for two-photon four-qubit systems with linear optics. Phys Rev A 2013;88:012302.

[47] Ren BC, Deng FG. Hyperentanglement purification and concentration assisted by diamond NV centers inside photonic crystal cavities. Laser Phys Lett 2013;10:115201.

[48] Ren BC, Long GL. General hyperentanglement con- 
centration for photon systems assisted by quantumdot spins inside optical microcavities. Opt Express 2014;22:6547-61.

[49] Ren BC, Long GL. Highly efficient hyperentanglement concentration with two steps assisted by quantum swap gates. Sci Rep 2015;5:16444.

[50] Li XH, Ghose S. Hyperconcentration for multipartite entanglement via linear optics. Laser Phys Lett 2014;11:125201.

[51] Li XH, Ghose S. Efficient hyperconcentration of nonlocal multipartite entanglement via the cross-Kerr nonlinearity. Opt Express 2015;23:3550-62.

[52] Li XH, Ghose S. Hyperentanglement concentration for time-bin and polarization hyperentangled photons. Phys Rev A 2015;91:062302.

[53] Cao C, Wang TJ, Mi SC, et al. Nonlocal hyperconcentration on entangled photons using photonic module system. Ann Phys 2016;369:128-38.

[54] Ren BC, Du FF, Deng FG. Two-step hyperentanglement purification with the quantum-state-joining method. Phys Rev A 2014;90:052309.

[55] Wang GY, Liu Q, Deng FG. Hyperentanglement purification for two-photon six-qubit quantum systems. Phys Rev A 2016;94:032319.

[56] Wang TJ, Liu LL, Zhang R, et al. One-step hyperentanglement purification and hyperdistillation with linear optics. Opt Express 2015;23:9284-94.

[57] Wang TJ, Wang C. High-efficient entanglement distillation from photon loss and decoherence. Opt Express 2015;23:31550-63.

[58] Ren BC, Wei HR, Deng FG. Deterministic photonic spatial-polarization hyper-controlled-not gate assisted by a quantum dot inside a one-side optical microcavity. Laser Phys Lett 2013;10:095202.

[59] Ren BC, Deng FG. Hyper-parallel photonic quantum computation with coupled quantum dots. Sci Rep 2014;4:4623.

[60] Ren BC, Wang GY, Deng FG. Universal hyperparallel hybrid photonic quantum gates with the dipole induced transparency in weak-coupling regime. Phys Rev A 2015;91:032328.

[61] Li T, Long GL. Hyperparallel optical quantum computation assisted by atomic ensembles embedded in double-sided optical cavities. Phys Rev A 2016;94:022343.

[62] Wei HR, Deng FG, Long GL. Hyper-parallel Toffoli gate on three-photon system with two degrees of freedom assisted by single-sided optical microcavities. Opt Express 2016;24:18619-30.

[63] Li XH, Deng FG, Zhou HY. Faithful qubit transmission against collective noise without ancillary qubits. Appl Phys Lett 2007;91:144101.

[64] Vaidman L, Yoran N. Methods for reliable teleportation. Phys Rev A 1999:59:116-25.

[65] Lütkenhaus N, Calsamiglia J, Suominen KA. Bell measurements for teleportation. Phys Rev A 1999;59:3295300 .

[66] Wang XL, Cai XD, Su ZE, et al. Quantum teleportation of multiple degrees of freedom of a single photon. Nature 2015;518:516-19.

[67] Bennett CH, Brassard G, Crepeau C, et al. Teleporting an unknown quantum state via dual classical and Einstein-Podolsky-Rosen channels. Phys Rev Lett 1993;70:1895-9.
[68] Walton ZD, Abouraddy AF, Sergienko AV, et al. Decoherence-free subspaces in quantum key distribution. Phys Rev Lett 2003;91:087901.

[69] Boileau JC, Gottesman D, Laflamme R, et al. Robust polarization-based quantum key distribution over a collective-noise channel. Phys Rev Lett 2004;92:017901.

[70] Boileau JC, Laflamme R, Laforest M, et al. Robust quantum communication using a polarization-entangled photon pair. Phys Rev Lett 2004;93:220501.

[71] Bennett CH, Bernstein HJ, Popescu S, et al. Concentrating partial entanglement by local operations. Phys Rev A 1996;53:2046-52.

[72] Zhao Z, Pan JW, Zhan MS. Practical scheme for entanglement concentration. Phys Rev A 2001;64:014301.

[73] Yamamoto T, Koashi M, Imoto N. Concentration and purification scheme for two partially entangled photon pairs. Phys Rev A 2001;64:012304.

[74] Sheng YB, Deng FG, Zhou HY. Nonlocal entanglement concentration scheme for partially entangled multipartite systems with nonlinear optics. Phys Rev A 2008;77:062325.

[75] Wang C. Efficient entanglement concentration for partially entangled electrons using a quantum-dot and microcavity coupled system. Phys Rev A 2012;86:012323.

[76] Bose S, Vedral V, Knight PL. Purification via entanglement swapping and conserved entanglement. Phys Rev A 1999;60:194-7.

[77] Shi BS, Jiang YK, Guo GC. Optimal entanglement purification via entanglement swapping. Phys Rev A 2000;62:054301.

[78] Sheng YB, Zhou L, Zhao SM, et al. Efficient single-photon-assisted entanglement concentration for partially entangled photon pairs. Phys Rev A 2012;85:012307.

[79] Deng FG. Optimal nonlocal multipartite entanglement concentration based on projection measurements. Phys Rev A 2012;85:022311.

[80] Du FF, Deng FG. Heralded entanglement concentration for photon systems with linear optical elements. Sci China Phys Mech Astro 2015;58:040303.

[81] Sheng Y, Pan J, Guo R, et al. Efficient $N$-particle W state concentration with different parity check gates. Sci China Phys Mech Astro 2015;58:060301.

[82] Wang C, Shen WW, Mi SC, et al. Concentration and distribution of entanglement based on valley qubits system in grapheme. Sci Bull 2015;60:2016-21.

[83] Zhao Z, Yang T, Chen YA, et al. Experimental realization of entanglement concentration and a quantum repeater. Phys Rev Lett 2003;90:207901.

[84] Yamamoto T, Koashi M, Ozdemir SK, et al. Experimental extraction of an entangled photon pair from two identically decohered pairs. Nature 2003;421:343-6.

[85] Sheng YB, Zhou L. Quantum entanglement concentration based on nonlinear optics for quantum communications. Entropy 2013;15:1776-820.

[86] Hong CK, Ou ZY, Mandel L. Measurement of subpicosecond time intervals between 2 photons by interference. Phys Rev Lett 1987;59:2044.

[87] Bennett CH, Brassard G, Popescu S, et al. Purification of noisy entanglement and faithful teleportation via noisy channels. Phys Rev Lett 1996;76:722-5.

[88] Deutsch D, Ekert A, Jozsa R, et al. Quantum privacy amplification and the security of quantum cryptography over noisy channels. Phys Rev Lett 1996;77:2818-21. 
[89] Pan JW, Simon C, Brukner Č, et al. Entanglement purification for quantum communication. Nature 2001;410:1067-70.

[90] Simon C, Pan JW. Polarization entanglement purification using spatial entanglement. Phys Rev Lett 2002;89:257901.

[91] Pan JW, Gasparoni S, Ursin R, et al. Experimental entanglement purification of arbitrary unknown states. Nature 2003;423:417-22.

[92] Sheng YB, Deng FG, Zhou HY. Efficient polarizationentanglement purification based on parametric downconversion sources with cross-Kerr nonlinearity. Phys Rev A 2008;77:042308.

[93] Wang C, Zhang Y, Jin GS. Polarization-entanglement purification and concentration using cross-Kerr nonlinearity. Quantum Inf Comput 2011;11:988-1002.

[94] Wang C, Zhang Y, Jin GS. Entanglement purification and concentration of electron-spin entangled states using quantum dot spins in optical microcavities. Phys Rev A 2011;84:032307.

[95] Sheng YB, Zhou L, Long GL. Hybrid entanglement purification for quantum repeaters. Phys Rev A 2013;88:022302.

[96] Zhou L, Sheng YB. Purification of logic-qubit entanglement. Sci Rep 2016;6:28813.

[97] Krenn M, Huber M, Fickler R, et al. Generation and confirmation of a $(100 \times 100)$-dimensional entangled quantum system. Proc Natl Acad Sci USA 2014;111:6243-7.

[98] Sheng YB, Zhou L. Deterministic polarization entanglement purification using time-bin entanglement. Laser Phys Lett 2014;11:085203.

[99] Sheng YB, Zhou L. Deterministic entanglement distillation for secure double-server blind quantum computation. Sci Rep 2015;5:7815.

[100] Vitelli C, Spagnolo N, Aparo L, et al. Joining the quantum state of two photons into one. Nat Photon 2013;7:521-6.

[101] Warburton RJ, Dürr CS, Karrai K, et al. Charged excitons in self-assembled semiconductor quantum dots. Phys Rev Lett 1997;79:5282-5.

[102] Hu CY, Ossau W, Yakovlev DR, et al. Optically detected magnetic resonance of excess electrons in type-I quantum wells with a low-density electron gas. Phys Rev B 1998;58:R1766-9.

[103] Hu CY, Young A, O'Brien JL, et al. Giant optical Faraday rotation induced by a single-electron spin in a quantum dot: applications to entangling remote spins via a single photon. Phys Rev B 2008;78:085307.

[104] Walls DF, Milburn GJ. Quantum optics. Berlin:Springer-Verlag, 1994.

[105] Hu CY, Munro WJ, O'Brien JL, et al. Proposed entanglement beam splitter using a quantum-dot spin in a double-sided optical microcavity. Phys Rev B 2009;80:205326.

[106] An JH, Feng M, Oh CH. Quantum-information processing with a single photon by an input-output process with respect to low- $Q$ cavities. Phys Rev A 2009;79:032303.

[107] Blais A, Huang RS, Wallraff A, et al. Cavity quantum electrodynamics for superconducting electrical circuits: An architecture for quantum computation. Phys Rev A 2004;69:062320.

[108] Wallraff A, Schuster DI, Blais A, et al. Strong coupling of a single photon to a superconducting qubit using cir- cuit quantum electrodynamics. Nature 2004;431:162-7.

[109] Blais A, Gambetta J, Wallraff A, et al. Quantuminformation processing with circuit quantum electrodynamics. Phys Rev A 2007;75:032329.

[110] DiCarlo L, Chow JM, Gambetta JM, et al. Demonstration of two-qubit algorithms with a superconducting quantum processor. Nature 2009;460:240-4.

[111] Chow JM, Gambetta JM, Magesan E, et al. Implementing a strand of a scalable fault-tolerant quantum computing fabric. Nat commun 2014;5:4015.

[112] Fedorov A, Steffen L, Baur M, et al. Implementation of a Toffoli gate with superconducting circuits. Nature 2012;481:170-2.

[113] Togan E, Chu Y, Trifonov AS, et al. Quantum entanglement between an optical photon and a solid-state spin qubit. Nature 2010;466:730-4.

[114] Yang WL, Yin ZQ, Xu ZY, et al. One-step implementation of multiqubit conditional phase gating with nitrogen vacancy centers coupled to a high- $Q$ silica microsphere cavity. Appl Phys Lett 2010;96:241113.

[115] Wei HR, Deng FG. Compact quantum gates on electronspin qubits assisted by diamond nitrogen-vacancy centers inside cavities. Phys Rev A 2013;88:042323.

[116] Neumann P, Kolesov R, Naydenov B, et al. Quantum register based on coupled electron spins in a roomtemperature solid. Nat Phys 2010;6:249-53.

[117] Li X, Wu Y, Steel D, et al. An all-optical quantum gate in a semiconductor quantum dot. Science 2003;301:80911 .

[118] Wei HR, Deng FG. Universal quantum gates for hybrid systems assisted by quantum dots inside double-sided optical microcavities. Phys Rev A 2013;87:022305.

[119] Wang HF, Zhu AD, Zhang S, et al. Optically controlled phase gate and teleportation of a controlled-NOT gate for spin qubits in a quantum-dot microcavity coupled system. Phys Rev A 2013;87:062337.

[120] Wei HR, Deng FG. Universal quantum gates on electron-spin qubits with quantum dots inside singleside optical microcavities. Opt Express 2014;22:593607.

[121] Bonato C, Haupt F, Oemrawsingh SSR, et al. CNOT and Bell-state analysis in the weak-coupling cavity QED regime. Phys Rev Lett 2010;104:160503.

[122] Xu GF, Zhang J, Tong DM, et al. Nonadiabatic holonomic quantum computation in decoherence-free subspaces. Phys Rev Lett 2012;109:170501.

[123] Gershenfeld NA, Chuang IL. Bulk spin-resonance quantum computation. Science 1997;275:350-6.

[124] Jones JA, Mosca M, Hansen RH. Implementation of a quantum search algorithm on a quantum computer. Nature 1998;393:344-6.

[125] Long GL, Xiao L. Experimental realization of a fetching algorithm in a 7-qubit NMR spin Liouville space computer. J Chem Phys 2003;119:8473-81.

[126] Feng G, Xu G, Long G. Experimental realization of nonadiabatic holonomic quantum computation. Phys Rev Lett 2013;110:190501.

[127] Duan LM, Kimble HJ. Scalable photonic quantum computation through cavity-assisted interactions. Phys Rev Lett 2004;92:127902.

[128] Koshino K, Ishizaka S, Nakamura Y. Deterministic photon-photon $\sqrt{S W A P}$ gate using a $\Lambda$ system. Phys Rev A 2010;82:010301(R). 
[129] Zhang J, Itzler MA, Zbinden H, et al. Advances in InGaAs/InP single-photon detector systems for quantum communication. Light Sci Appl 2015;4:e286.

[130] Qin ZZ, Prasad AS, Brannan T, et al. Complete temporal characterization of a single photon. Light Sci Appl 2015;4:e298.

[131] Knill E, Laflamme R, Milburn GJA. A scheme for efficient quantum computation with linear optics. Nature 2001;409:46-52.

[132] Pittman TB, Jacobs BC, Franson JD. Probabilistic quantum logic operations using polarizing beam splitters. Phys Rev A 2001;64:062311.

[133] Gasparoni S, Pan JW, Walther P, et al. Realization of a photonic controlled-not gate sufficient for quantum computation. Phys Rev Lett 2004;93:020504.

[134] Zhao Z, Zhang AN, Chen YA, et al. Experimental demonstration of a nondestructive controlled-NOT quantum gate for two independent photon qubits. Phys Rev Lett 2005;94:030501.

[135] Browne DE, Rudolph T. Resource-efficient linear optical quantum computation. Phys Rev Lett 2005;95:010501.

[136] Gong YX, Guo GC, Ralph TC. Methods for a linear optical quantum Fredkin gate. Phys Rev A 2008;78:012305.

[137] Xu JS, Li CF. Quantum integrated circuit: classical characterization. Sci Bull 2015;60:141

[138] Nemoto K, Munro WJ. Nearly deterministic linear optical controlled-not gate. Phys Rev Lett 2004;93:250502.

[139] Duan LM, Kimble HJ. Scalable photonic quantum computation through cavity-assisted interactions. Phys Rev Lett 2004;92:127902.

[140] Wei HR, Deng FG. Scalable photonic quantum computing assisted by quantum-dot spin in double-sided optical microcavity. Opt Express 2013;21:17671-85.

[141] Yoran N, Reznik B. Deterministic linear optics quantum computation with single photon qubits. Phys Rev Lett 2003;91:037903.

[142] Fiorentino M, Wong FNC. Deterministic controlled-
NOT gate for single-photon two-qubit quantum logic. Phys Rev Lett 2004;93:070502.

[143] Fiorentino M, Kim T, Wong FNC. Single-photon twoqubit SWAP gate for entanglement manipulation. Phys Rev A 2005;72:012318.

[144] Scholz M, Aichele T, Ramelow S, et al. Deutsch-Jozsa algorithm using triggered single photons from a single quantum dot. Phys Rev Lett 2006;96:180501.

[145] Zhang P, Liu RF, Huang YF, et al. Demonstration of Deutsch's algorithm on a stable linear optical quantum computer. Phys Rev A 2010;82:064302.

[146] Abouraddy AF, Di Giuseppe G, Yarnall TM, et al. Implementing one-photon three-qubit quantum gates using spatial light modulators. Phys Rev A 2012;86:050303(R).

[147] Gao WB, Xu P, Yao XC, et al. Experimental realization of a controlled-NOT gate with four-photon six-qubit cluster states. Phys Rev Lett 2010;104:020501.

[148] Vallone G, Pomarico E, Martini FD, et al. Active oneway quantum computation with two-photon four-qubit cluster states. Phys Rev Lett 2008;100:160502.

[149] Abouraddy AF, Giuseppe GD, Yarnall TM, et al. Implementing one-photon three-qubit quantum gates using spatial light modulators. Phys Rev A 2012;86:050303.

[150] Zheng C, Long GF. Quantum secure direct dialogue using Einstein-Podolsky-Rosen pairs. Sci China Phys Mech Astro 2014;57:1238-43.

[151] Cao DY, Liu BH, Wang Z, et al. Multiuser-tomultiuser entanglement distribution based on $1550 \mathrm{~nm}$ polarization-entangled photons. Sci Bull 2015;60:11281132.

[152] Bermudez A, Martin-Delgado MA. Hyper-entanglement in a relativistic two-body system. J Phys A Math Theor 2008;41:485302.

[153] Liu K, Guo J, Cai CX, et al. Experimental generation of continuous-variable hyperentanglement in an optical parametric oscillator. Phys Rev Lett 2014;113:170501. 